Electronic Supporting Information

\title{
Palladium(II), Ruthenium(II), and Ruthenium(III) Complexes of 23-Thiaazuliporphyrin: The Case of Coordination-Induced Contraction
}

Michał J. Białek and Lechosław Latos-Grażyński*

Department of Chemistry, University of Wrockaw, 14 F. Joilot-Curie Street, 50-383 Wrocław, Poland *(LLG) lechoslaw.latos-grazynski@chem.uni.wroc.pl; http://llg.chem.uni.wroc.pl/ 


\section{Table of contents}

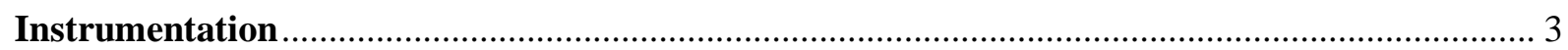

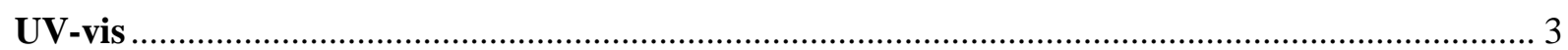

Figure S1. Protonation of 23-thiaazuliporphyrin with TFA ............................................................. 3

Figure S2. Metal complexes: 1-Pd(II) ${ }^{+}, \mathbf{1 - R u}(\mathrm{II}), \mathbf{1}-\mathrm{Ru}(\mathrm{II})^{+}, \mathbf{1}-\mathrm{Ru}(\mathrm{III}), \mathbf{2}-\mathrm{Pd}(\mathrm{II})$, and 2a-Pd(II) ........ 4

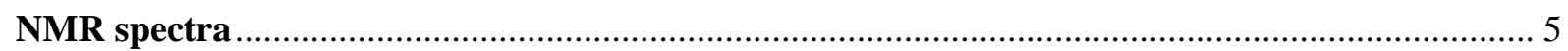

Figure S3. Protonation of 23-thiaazuliporphyrin with TFA …......................................................... 5

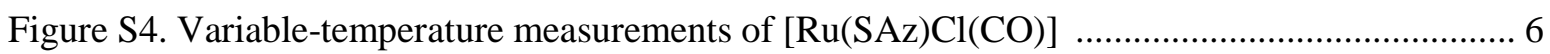

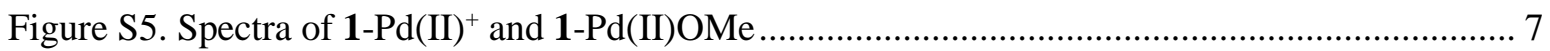

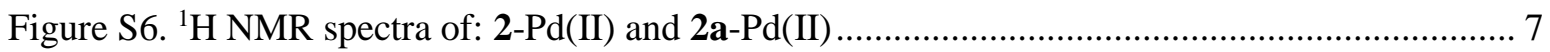

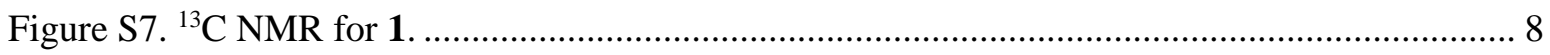

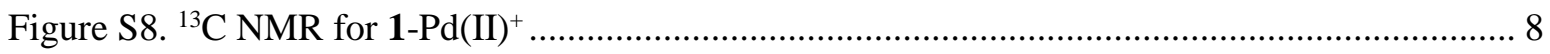

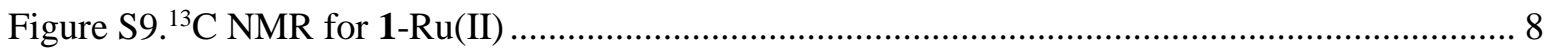

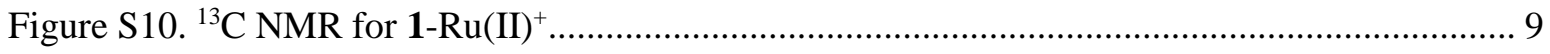

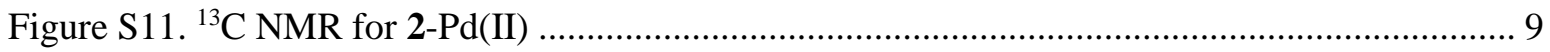

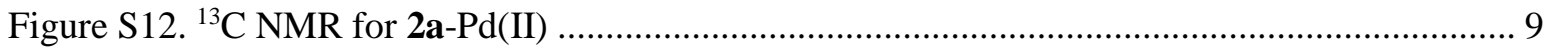

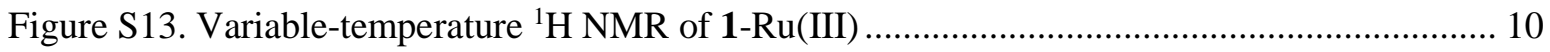

Figure S14. Comparison of ${ }^{1} \mathrm{H}$ NMR spectra of $1-\mathrm{Ru}(\mathrm{III})$ and $1-\mathrm{Ru}(\mathrm{III})-d_{2}$ derivative .................... 10

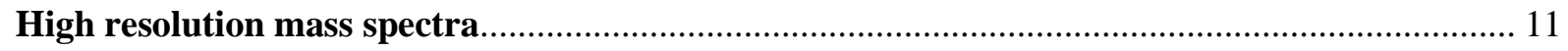

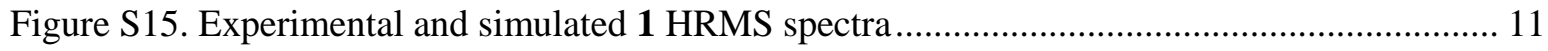

Figure S16. Experimental and simulated 1-Pd(II) ${ }^{+}$HRMS spectra.................................................. 11

Figure S17. Experimental and simulated 1-Ru(II) HRMS spectra................................................. 11

Figure S18. Experimental and simulated 1-Ru(III) HRMS spectra ............................................... 12

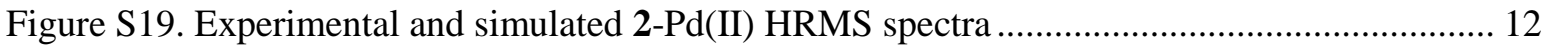

Figure S20. Experimental and simulated 2a-Pd(II) HRMS spectra .............................................. 12

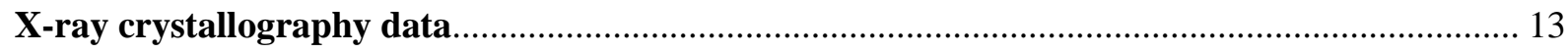

Table S1.Crystal and structure refinement data for 1, 1-Ru(II), 1-Pd(II) ${ }^{+}$, and $\mathbf{2 a}-\mathrm{Pd}(\mathrm{II}) \ldots \ldots \ldots \ldots \ldots \ldots . . . . .13$

Figure S21. Selected bond distances for 1, 1-Ru(II), 1-Pd(II) ${ }^{+}$, and $\mathbf{2 a - P d ( I I ) ~ . . . . . . . . . . . . . . . . . . . . . . . . . . . . . . . . ~} 14$

Figure $\mathrm{S} 22$. Imposed space filling structures of $1-\mathrm{Ru}(\mathrm{II})$ and $\left[\mathrm{Ru}(\mathrm{TPAP})(\mathrm{CO})\left\{\mathrm{Ru}_{4}(\mathrm{CO})_{9}\right\}\right] \ldots \ldots \ldots . . .15$

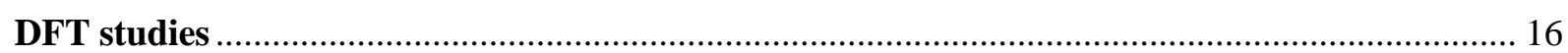

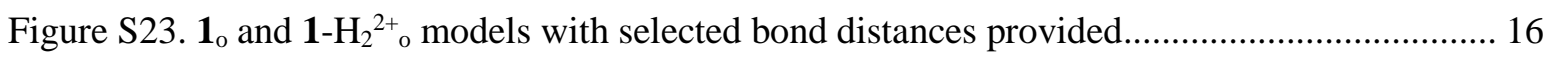

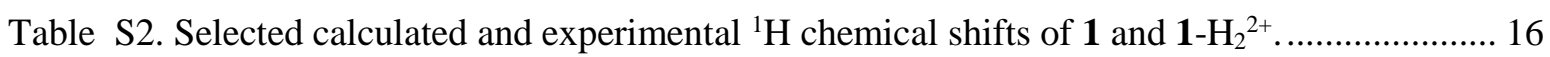

Figure S24. Correlation diagrams between calculated and experimental shifts for $\mathbf{1}$ and $\mathbf{1}-\mathrm{H}_{2}{ }^{2+} \ldots . .16$

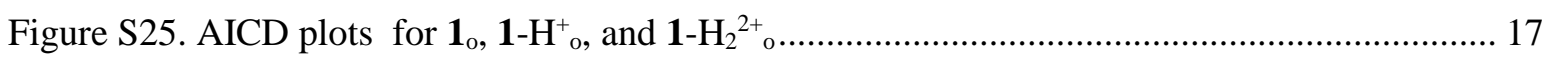

Figure S26. Bond distances for models 1-Ru(II) $)_{0}, 1-\mathrm{Ru}(\mathrm{II})^{+}{ }_{\mathrm{o}}, \mathbf{1 - P d}(\mathrm{II})^{+}{ }_{\mathrm{o}}$, and 1-Pd(II)OMe $\mathrm{OM}_{\mathrm{o}} \ldots \ldots \ldots . . . .17$

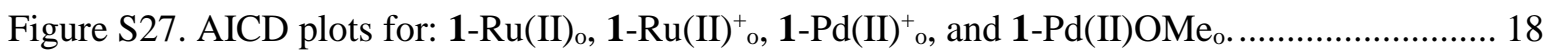




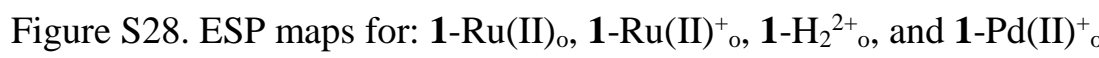
18

Table S3. Hirshfeld partial atomic charges on heavy atoms for $\mathbf{1}-\mathrm{Ru}(\mathrm{II})_{\mathrm{o}}$ and $\mathbf{1}-\mathrm{Ru}(\mathrm{II})^{+}{ }_{\mathrm{o}}$ 19

Figure S29. Selected bond distances for models: 1-Ru(II)- $1_{o}, 1-\mathrm{Ru}(\mathrm{II})-t_{o}$, and 1-Ru(II)- $2_{o}$ 20

Figure S30. 1-Ru(II)- $t_{o}$ model showing one imaginary frequency vibration.................................... 20

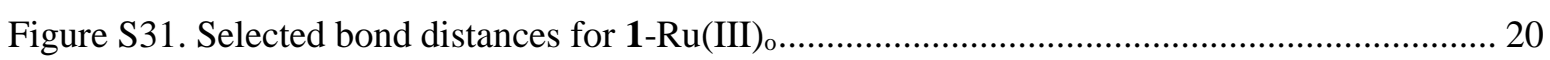

Figure S32. Selected bond distances for models: 1-Ru(III $)_{0}-$ EGS and 1-Ru(III) $)_{0}-$ EES ............... 21

Table S4. Partial atomic spin densities on heavy atoms for 1-Ru(III) $)_{0}-$ EGS and - EES .............. 21

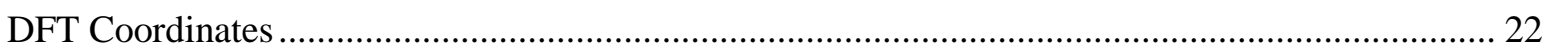




\section{Instrumentation}

NMR spectra were measured on Bruker Avance $500 \mathrm{MHz}$ and Bruker Avance III $600 \mathrm{MHz}$ spectrometers. ${ }^{1} \mathrm{H}$ and ${ }^{13} \mathrm{C}$ shifts were referenced to the residual resonances of deuterated solvents. Infrared absorption spectra were measured by the ATR technique. Absorption spectra were recorded on a Varian Carry-50 Bio spectrophotometer. Mass spectra (High Resolution and Accurate Mass) were recorded on a Bruker micrOTOF-Q spectrometer using the electrospray technique.

\section{UV-vis}

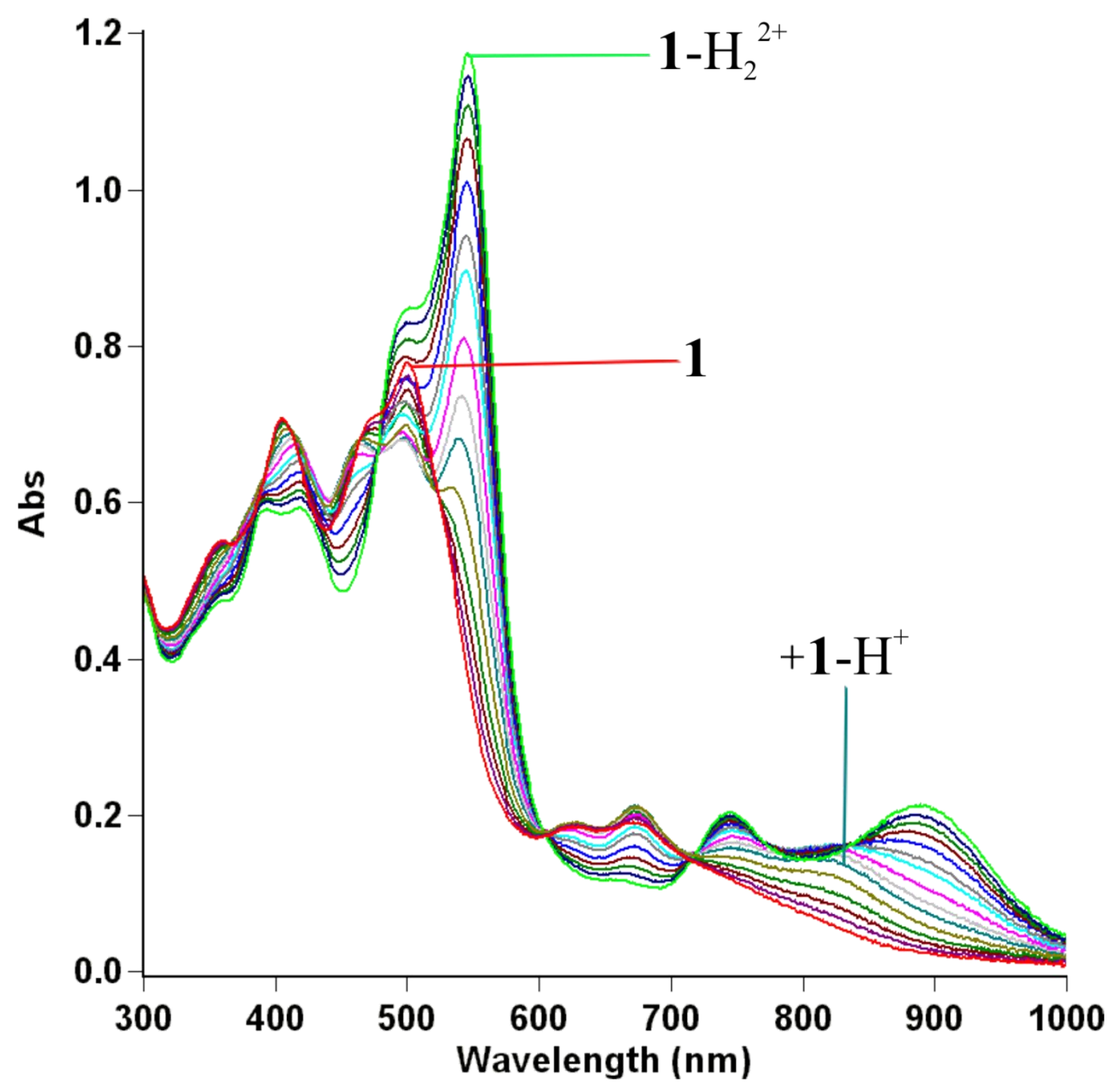

Figure S1. Electronic spectra of gradual protonation of 23-thiaazuliporphyrin with TFA $\left(\mathrm{CHCl}_{3}, 298 \mathrm{~K}\right)$. One can see a transitional spectrum with maxima that come neither from 1 nor $1-\mathrm{H}_{2}^{2+}$. 
a)

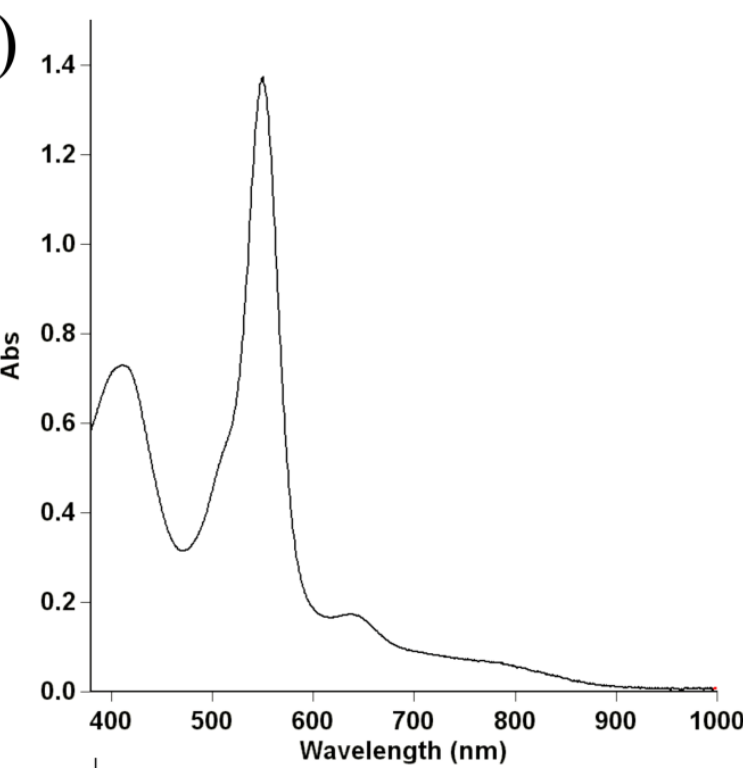

c)

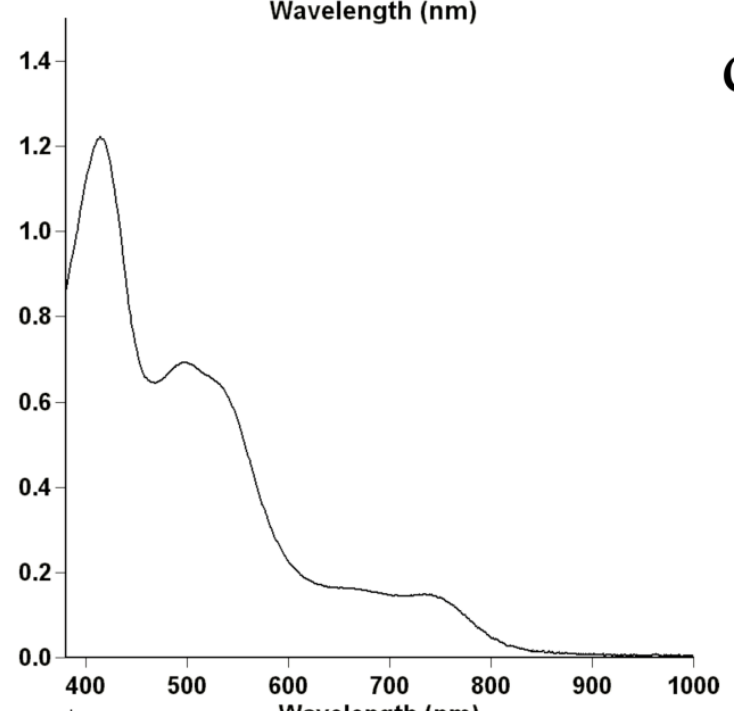

e)

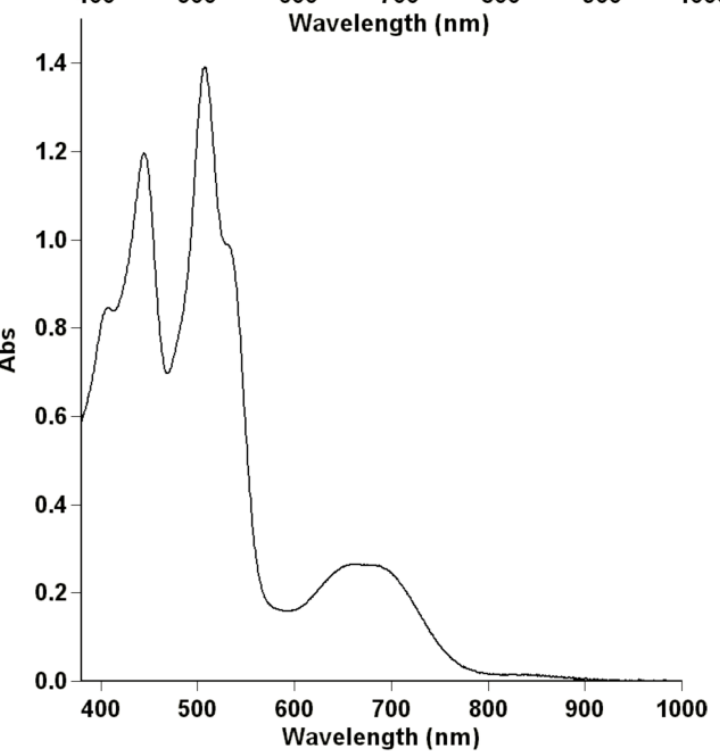

b)

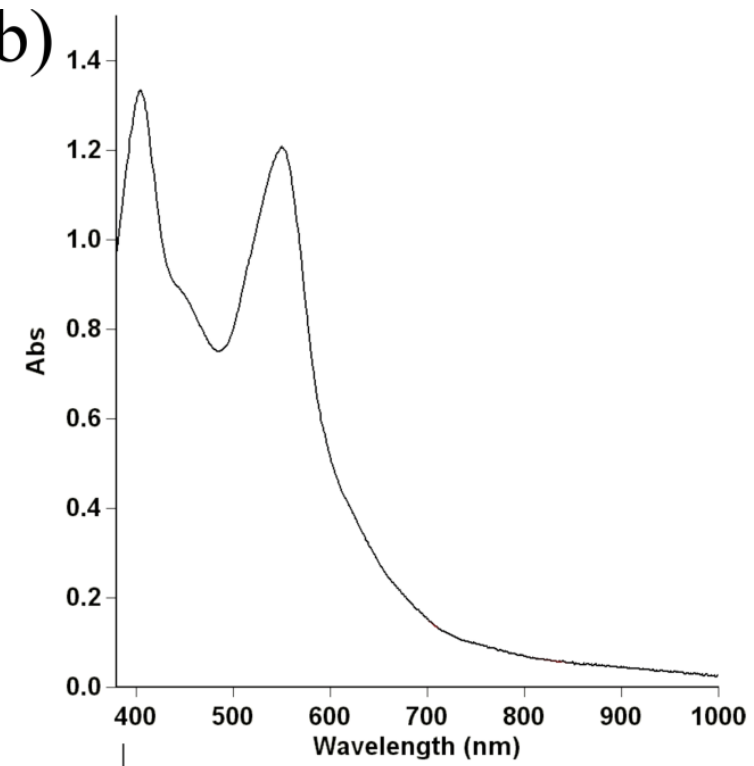

d)

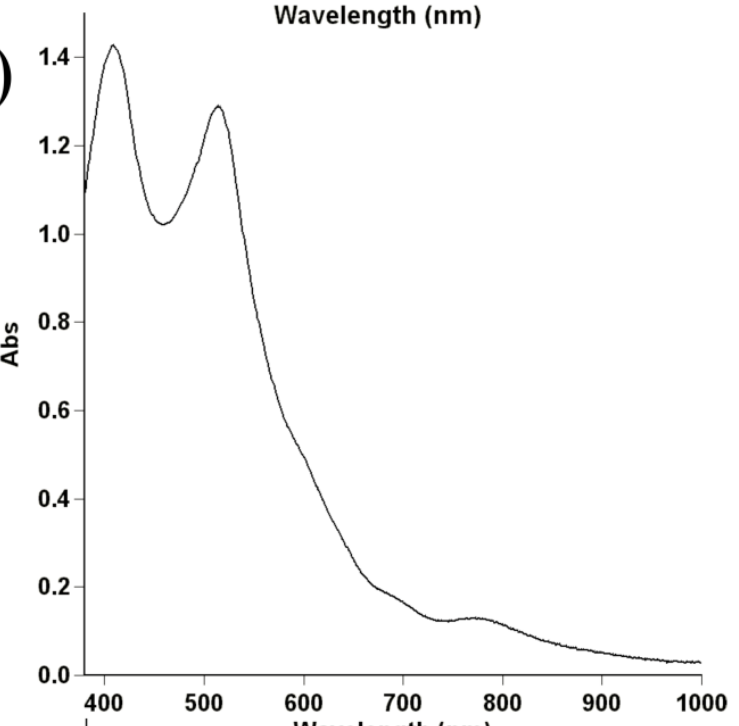

f)

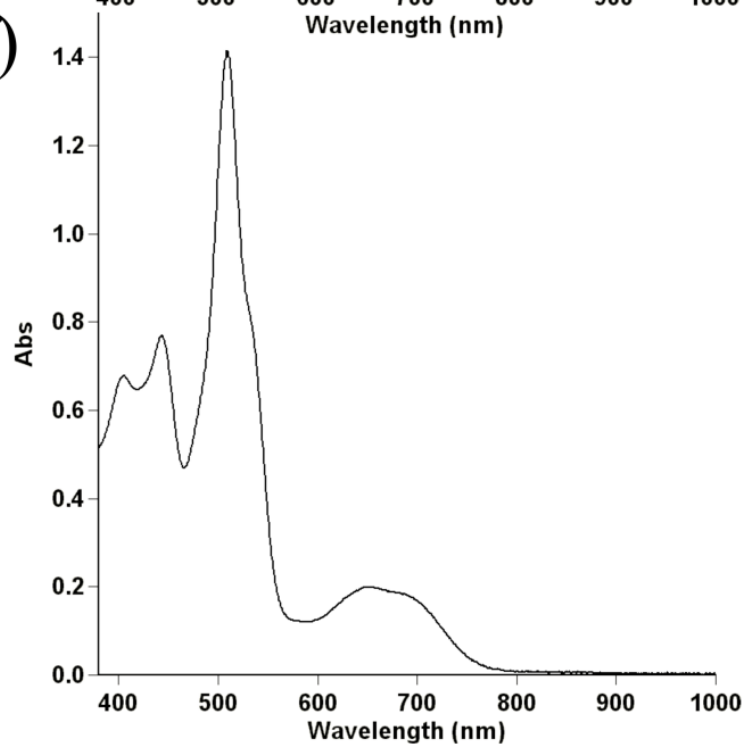

Figure S2. Electronic spectra $\left(\mathrm{CHCl}_{3}, 298 \mathrm{~K}\right)$ of metal complexes: a) 1-Pd(II $)^{+}$, b) 1-Ru(II), c) 1-Ru(II) ${ }^{+}$, d) 1-Ru(III), e) 2$\mathrm{Pd}(\mathrm{II})$, and f) 2a-Pd(II) 


\section{NMR spectra}

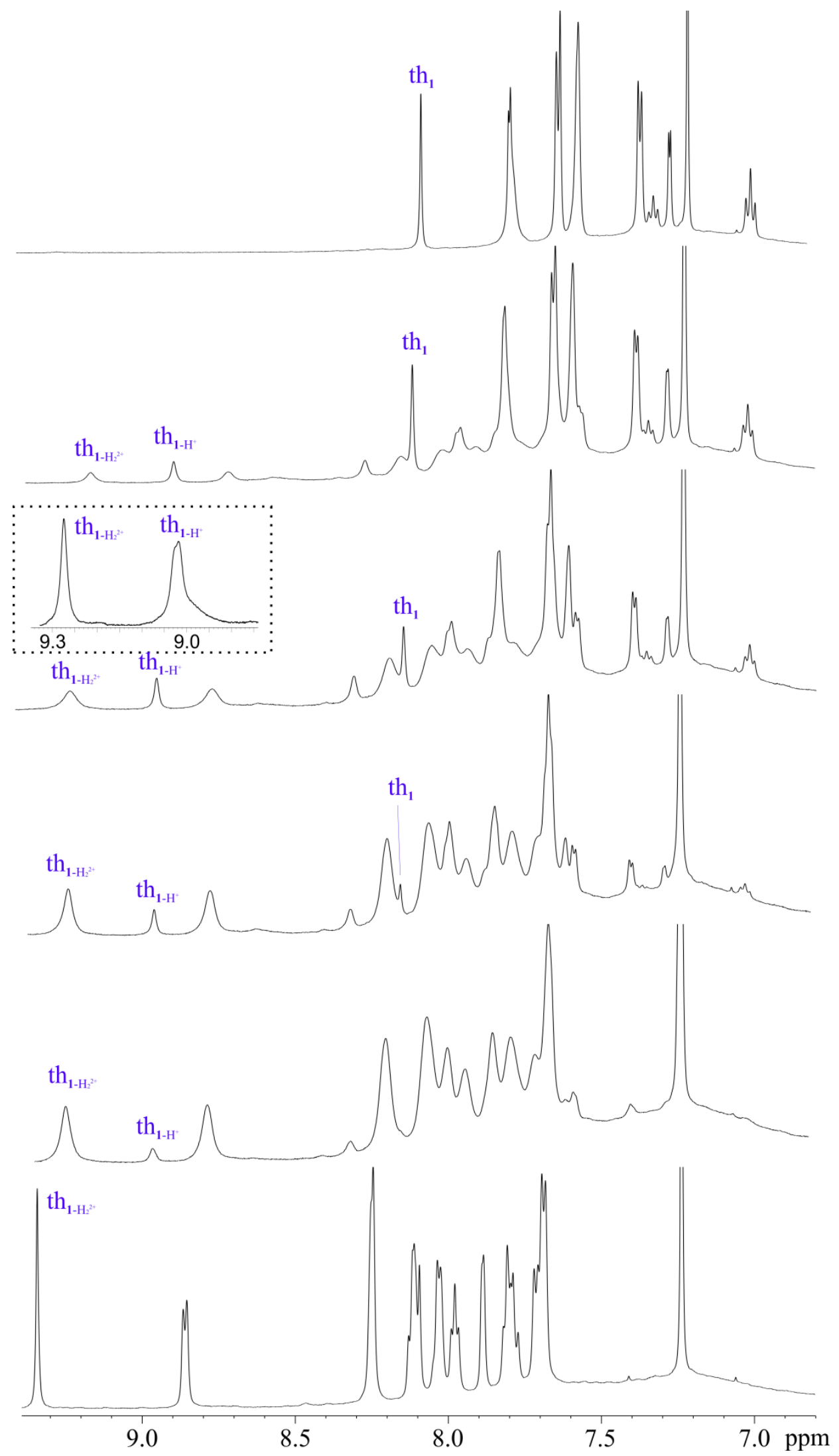

Figure S3. Protonation of 23-thiaazuliporphyrin with TFA (600 MHz, $\left.\mathrm{CDCl}_{3}, 210 \mathrm{~K}\right)$. The top spectrum - free 1, the bottom one $-1-\mathrm{H}_{2}{ }^{2+}$. Inset shows thiophene signals from a mixture of $1-\mathrm{H}^{+}$and $1-\mathrm{H}_{2}{ }^{2+}$ at $185 \mathrm{~K}$ in $\mathrm{CD}_{2} \mathrm{Cl}_{2}$. 


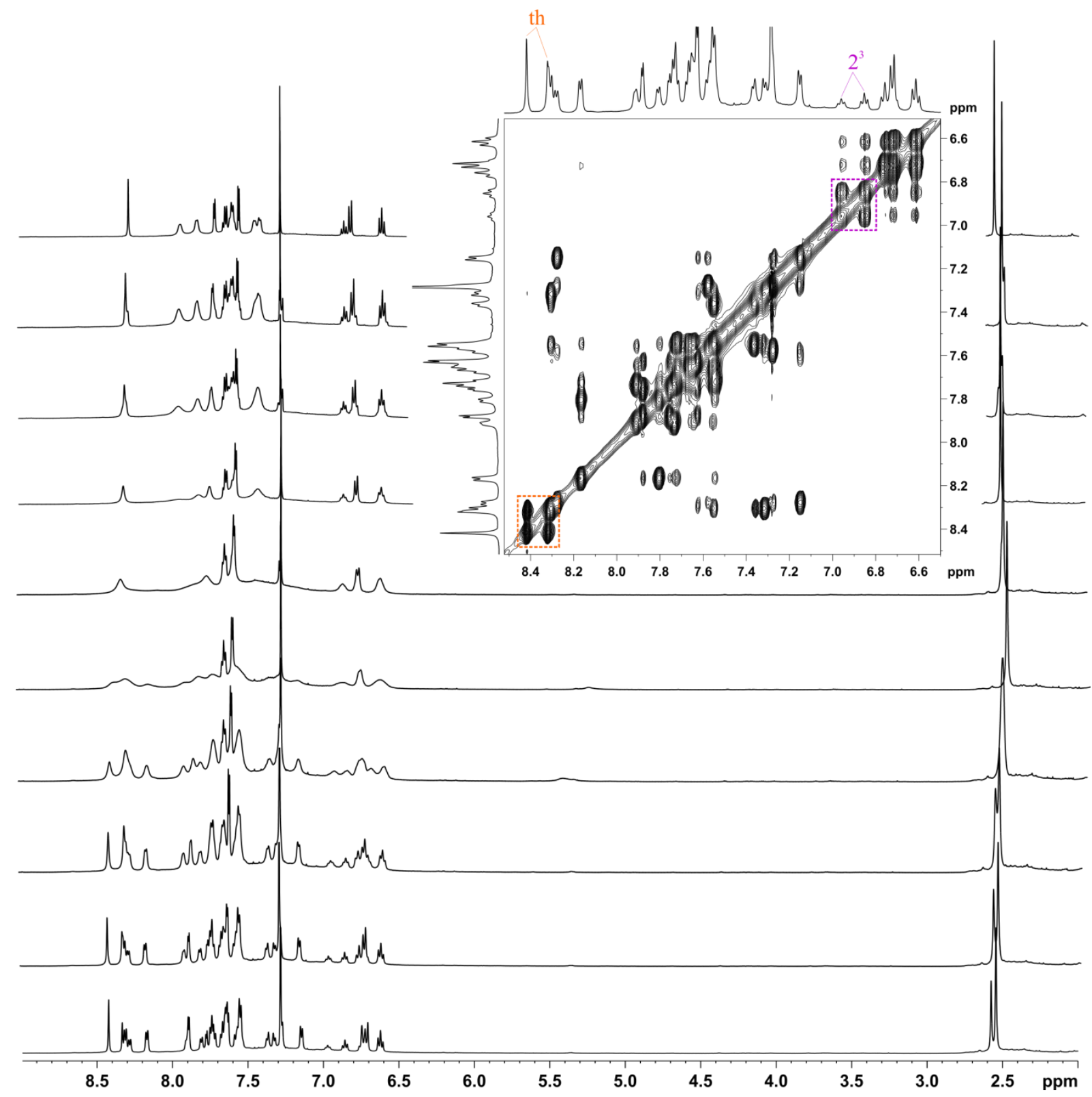

Figure S4. Variable-temperature measurements of $[\mathrm{Ru}(\mathrm{SAz}) \mathrm{Cl}(\mathrm{CO})]$ from $300 \mathrm{~K}$ (top) to $210 \mathrm{~K}$ (bottom) (600 MHz, $\left.\mathrm{CDCl}_{3}\right)$. The inset presents NOESY spectrum taken at $220 \mathrm{~K}$ that shows EXSY cross-peaks, with two selected from thiophene and $2^{3}$ azulene marked orange and pink, respectively. The coalescence temperature $\left(\mathrm{T}_{\mathrm{c}}\right)$ is estimated to be $255 \mathrm{~K}$. Energy barrier $\left(\Delta \mathrm{G}^{\ddagger}\right)$ was calculated from equation: $\Delta \mathrm{G}^{\ddagger}=\mathrm{aT}_{\mathrm{c}}\left[9.972+\log \left(\frac{\mathrm{T}_{\mathrm{c}}}{\Delta \mathbf{v}}\right)\right]$, where a $=4.575 \cdot 10^{-3}$ (when calculating energy in $\mathrm{kcal} / \mathrm{mol}$ ) and $\Delta v$ (here $54 \mathrm{~Hz}$ for thiophene) is a peak separation in the low temperature (in $\mathrm{Hz}$ ). The equation is derived from the Eyring equation after assumption of rate constant $\mathrm{k}_{\text {coal }}=\pi \Delta v / \sqrt{2}$ (implying equal populations of states, thus this is only a rough estimation). 


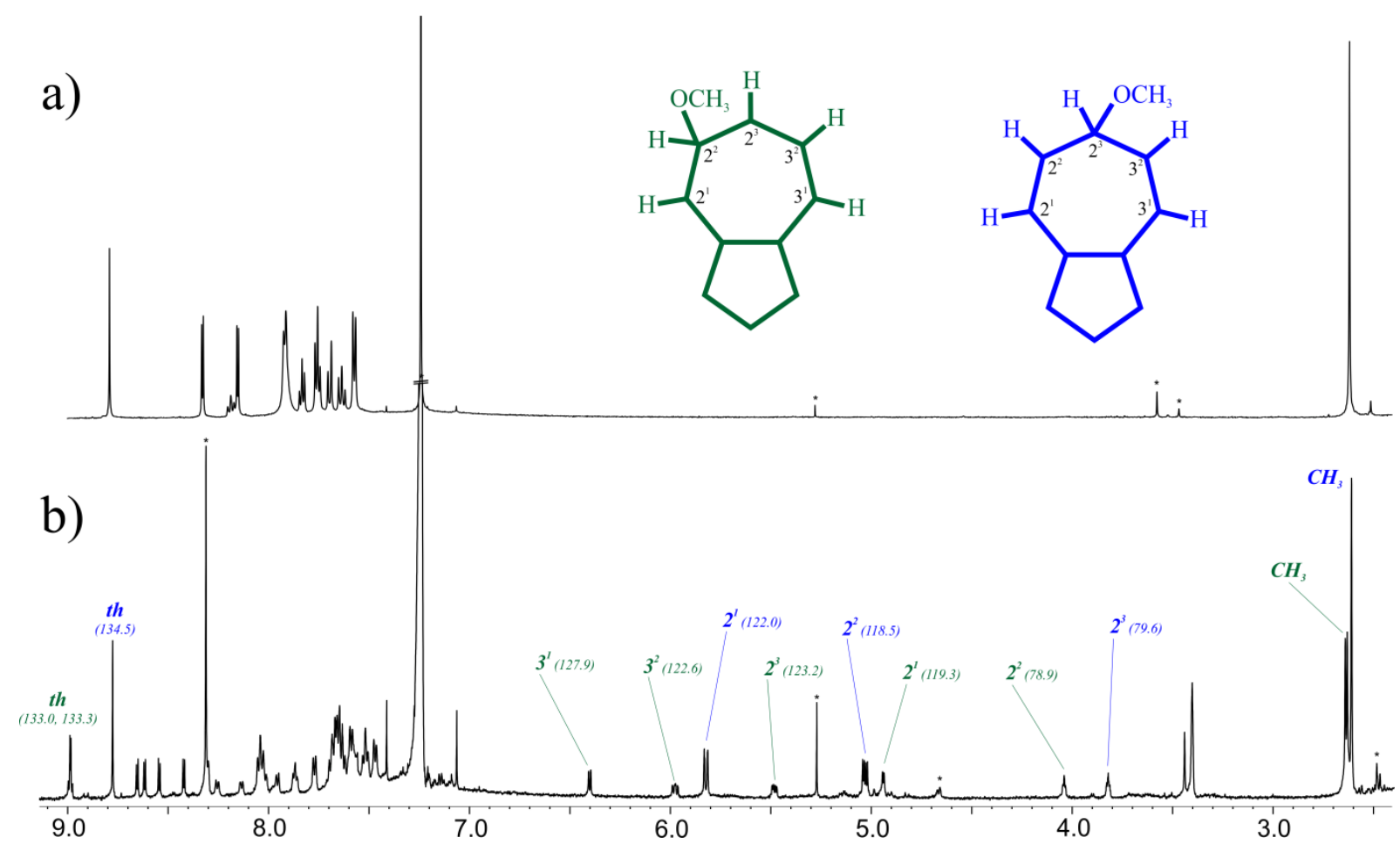

Figure S5. ${ }^{1} \mathrm{H}$ NMR spectra $\left(600 \mathrm{MHz}, \mathrm{CDCl}_{3}, 300 \mathrm{~K}\right)$ of a) 1 - $\mathrm{Pd}(\mathrm{II})^{+}$and b) 1-Pd(II) ${ }^{+}$after addition of $\mathrm{NaOCH}_{3}$ solution (1$\mathrm{Pd}(\mathrm{II}) \mathrm{OMe}$ ) in $\mathrm{CH}_{3} \mathrm{OH}$ at $210 \mathrm{~K}$ and waiting for several hours at room temperature. Selected signals from two $\mathrm{CH}_{3} \mathrm{O}^{-}$adducts are marked blue and green. In brackets are values of ${ }^{13} \mathrm{C}$ shifts taken from a HSQC correlation spectrum.

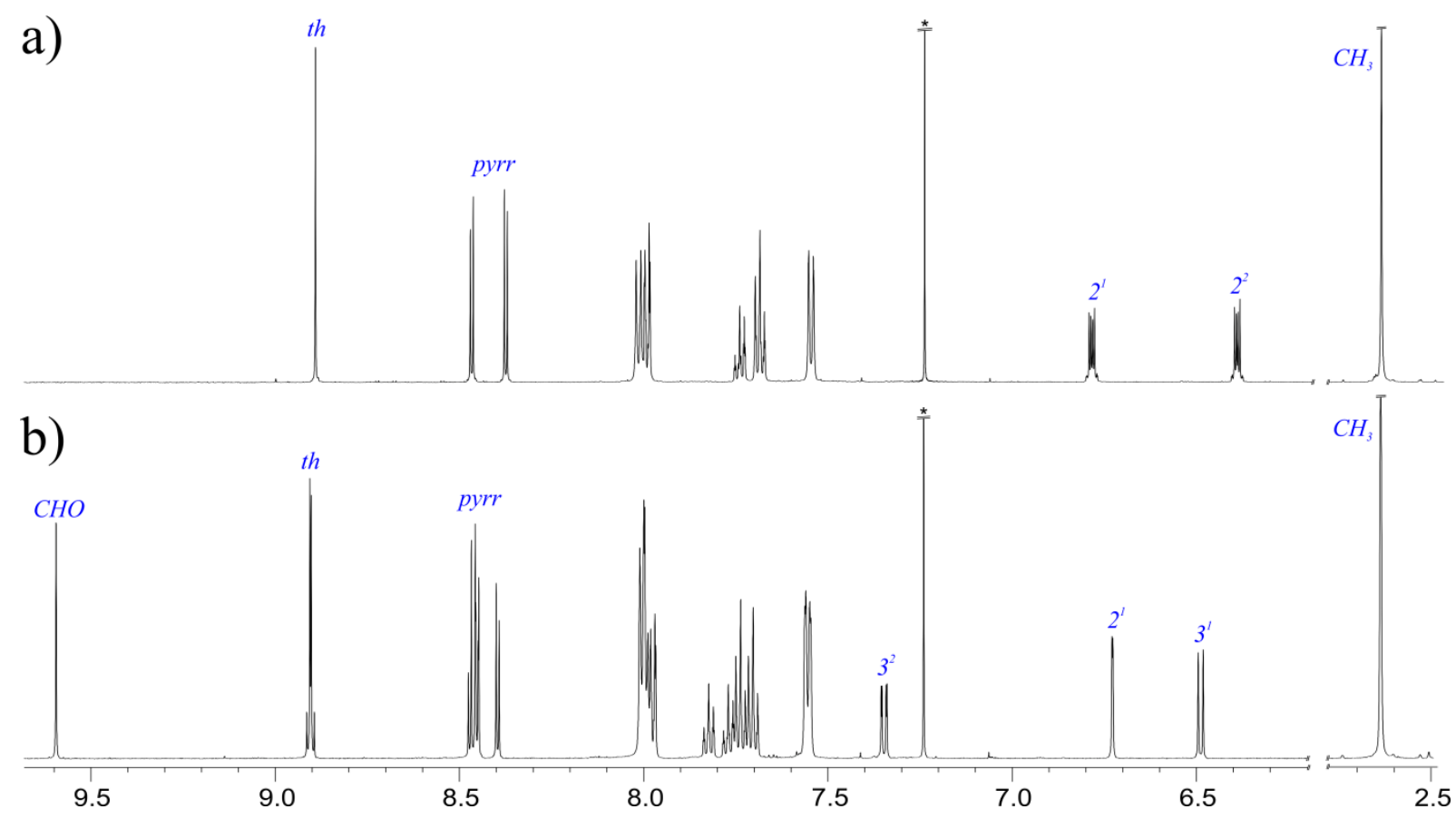

Figure S6. ${ }^{1} \mathrm{H}$ NMR spectra (600 MHz, $\left.\mathrm{CDCl}_{3}, 300 \mathrm{~K}\right)$ of: a) 2-Pd(II) and b) 2a-Pd(II) 


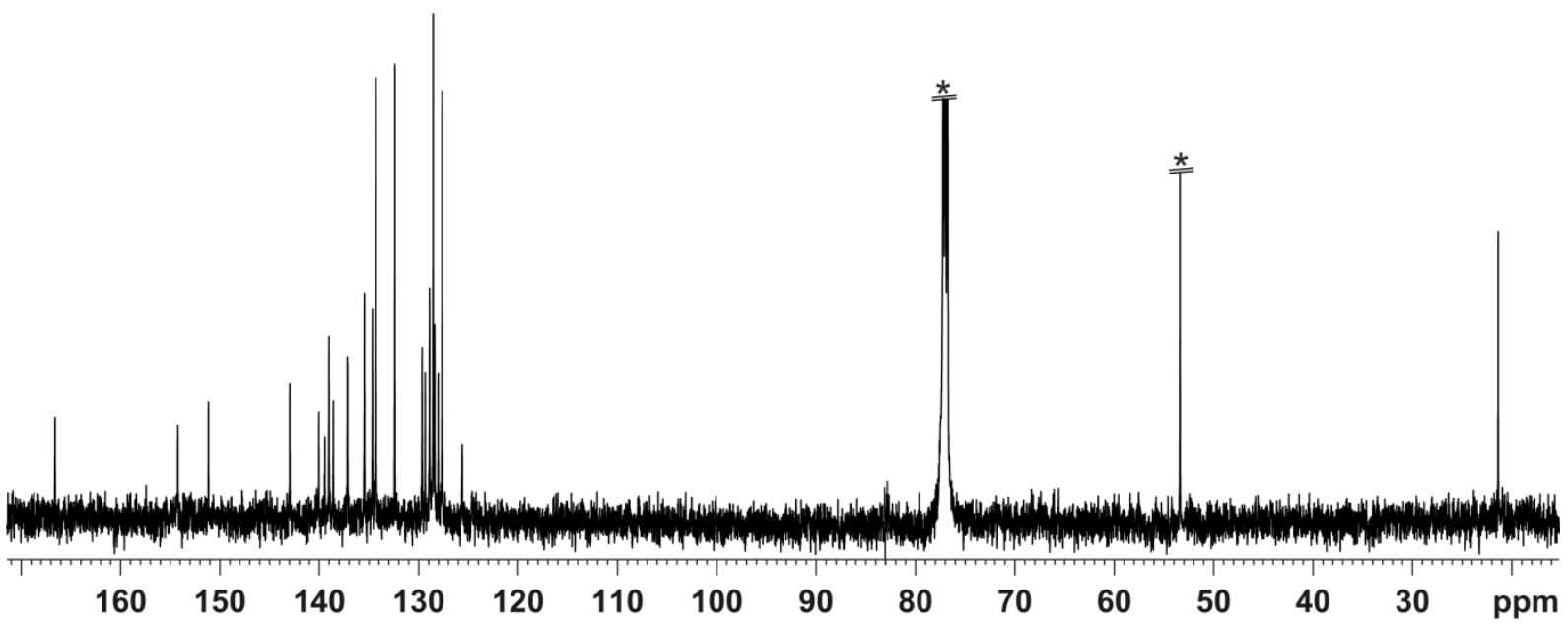

Figure S7. ${ }^{13} \mathrm{C}$ NMR for $\mathbf{1}\left(\mathrm{CDCl}_{3}, 151 \mathrm{MHz}, 300 \mathrm{~K}\right)$.

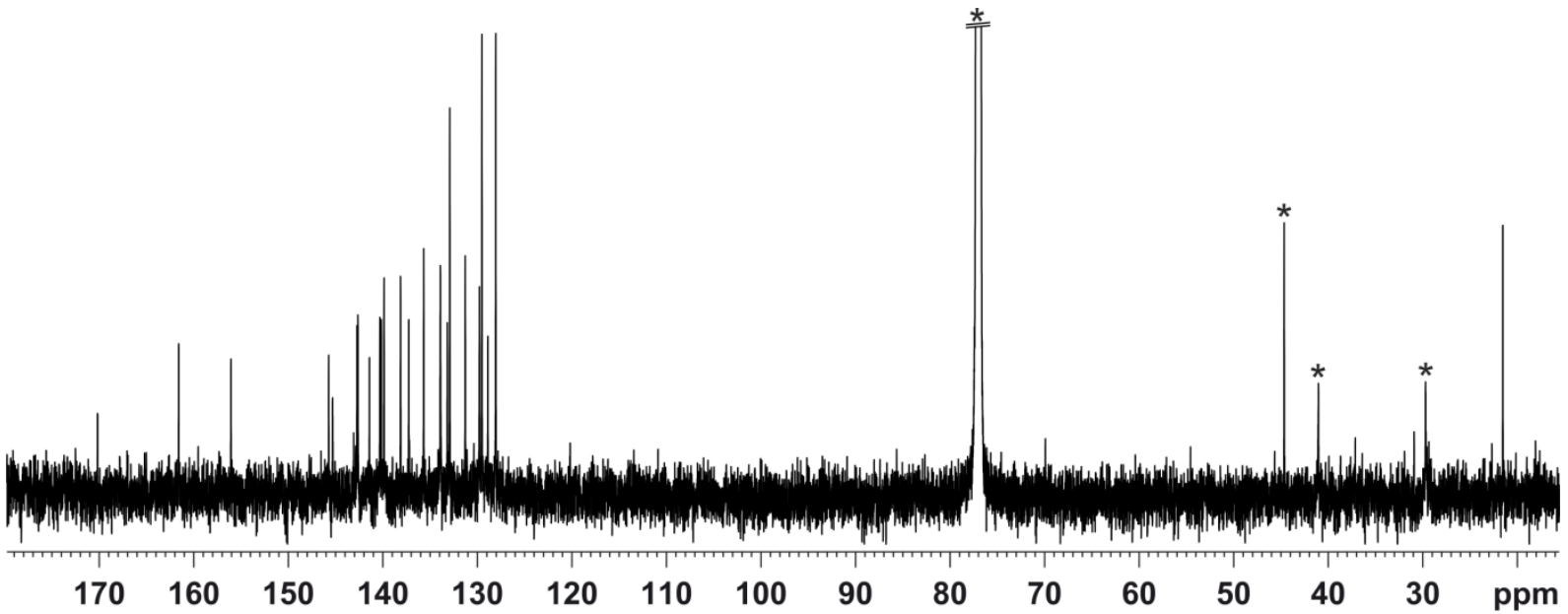

Figure S8. ${ }^{13} \mathrm{C}$ NMR for $1-\mathrm{Pd}(\mathrm{II})^{+}\left(\mathrm{CDCl}_{3}, 151 \mathrm{MHz}, 300 \mathrm{~K}\right)$.

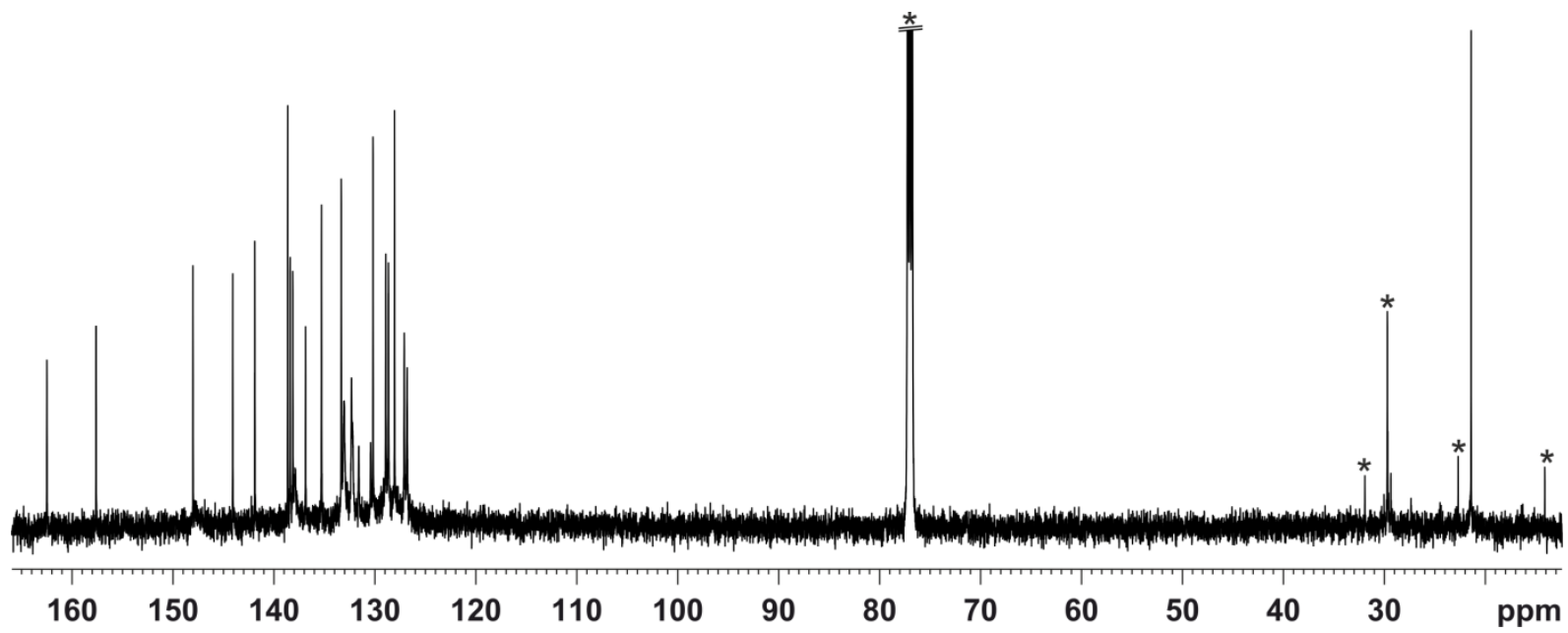

Figure S9. ${ }^{13} \mathrm{C}$ NMR for $1-\mathrm{Ru}(\mathrm{II})\left(\mathrm{CDCl}_{3}, 151 \mathrm{MHz}, 300 \mathrm{~K}\right)$. 


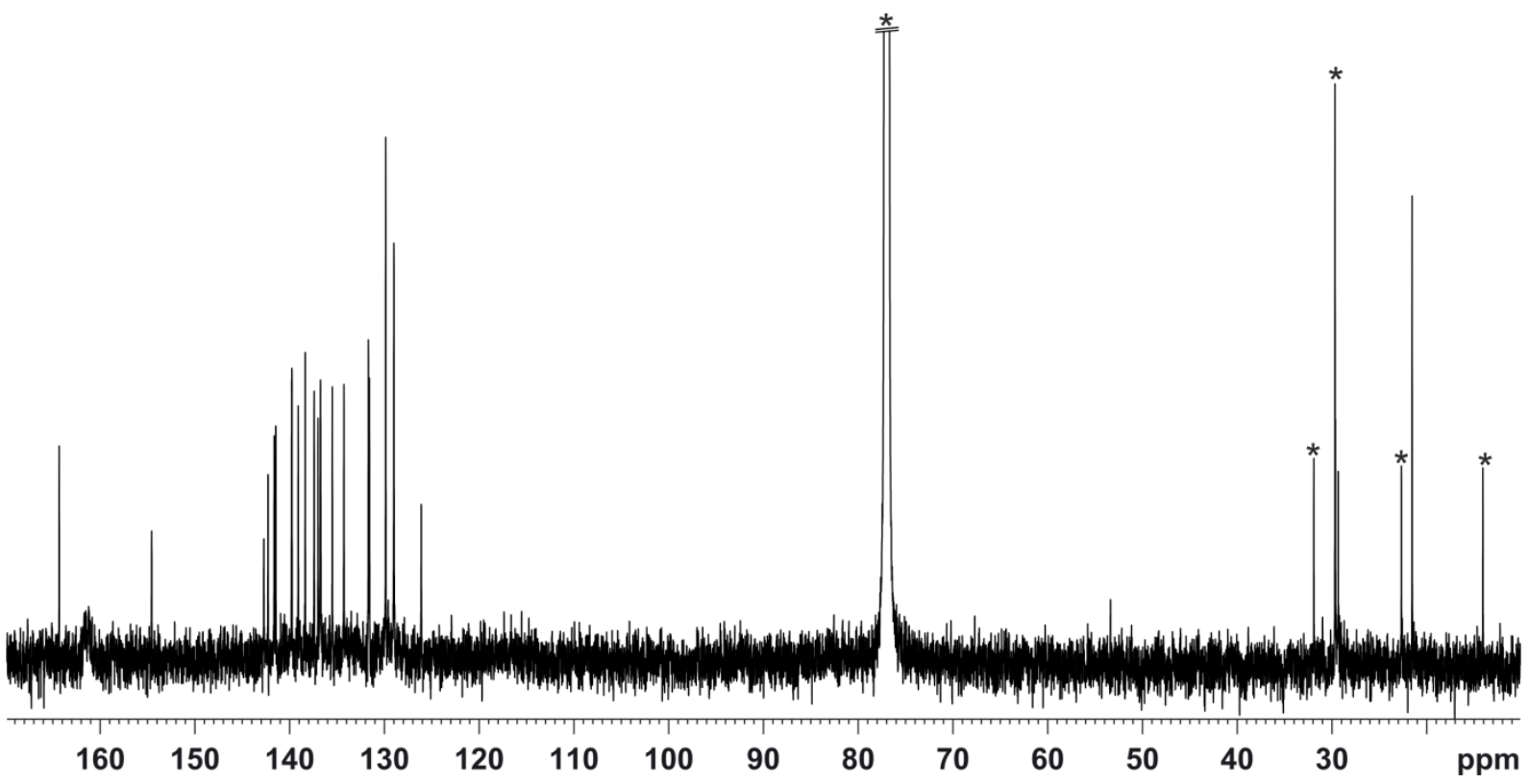

Figure S10. ${ }^{13} \mathrm{C} \mathrm{NMR}$ for $1-\mathrm{Ru}(\mathrm{II})^{+}\left(\mathrm{CDCl}_{3}, 151 \mathrm{MHz}, 300 \mathrm{~K}\right)$.

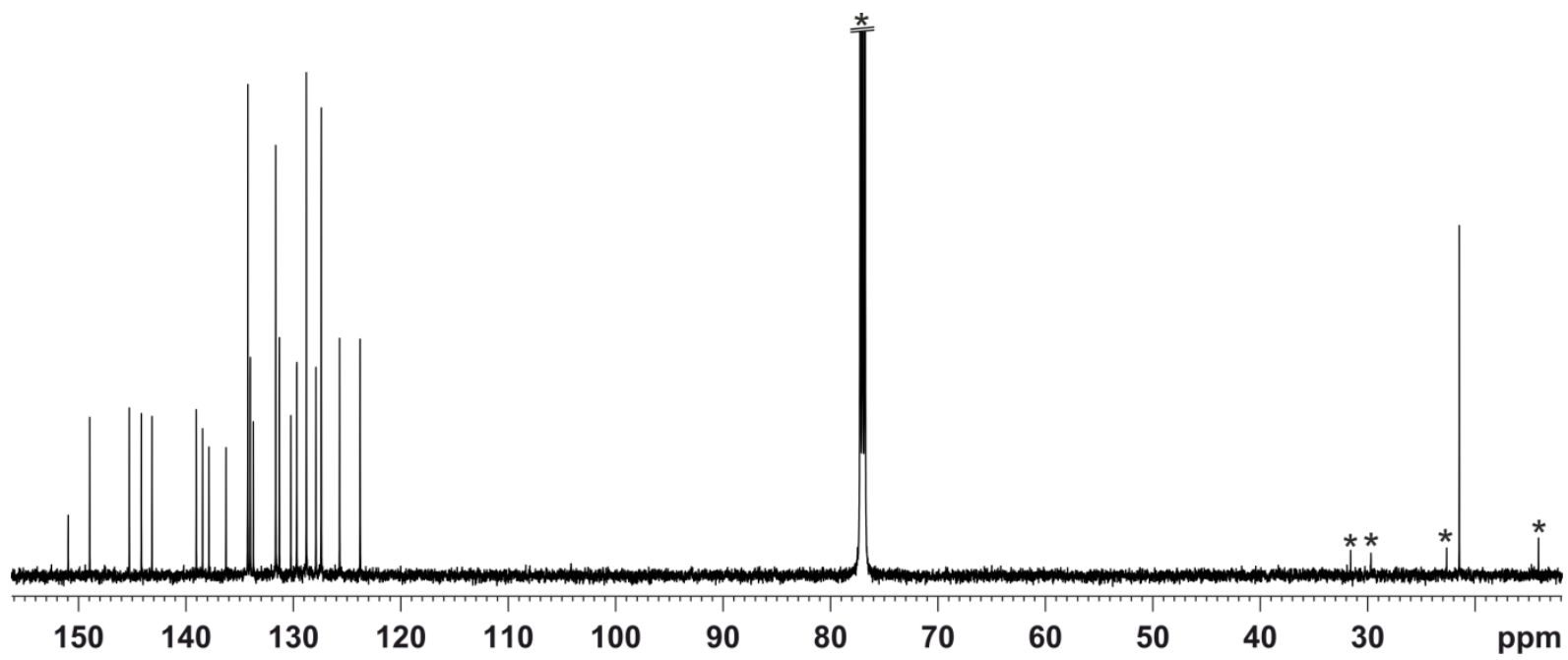

Figure S11. ${ }^{13} \mathrm{C}$ NMR for 2 -Pd(II) $\left(\mathrm{CDCl}_{3}, 151 \mathrm{MHz}, 300 \mathrm{~K}\right)$.

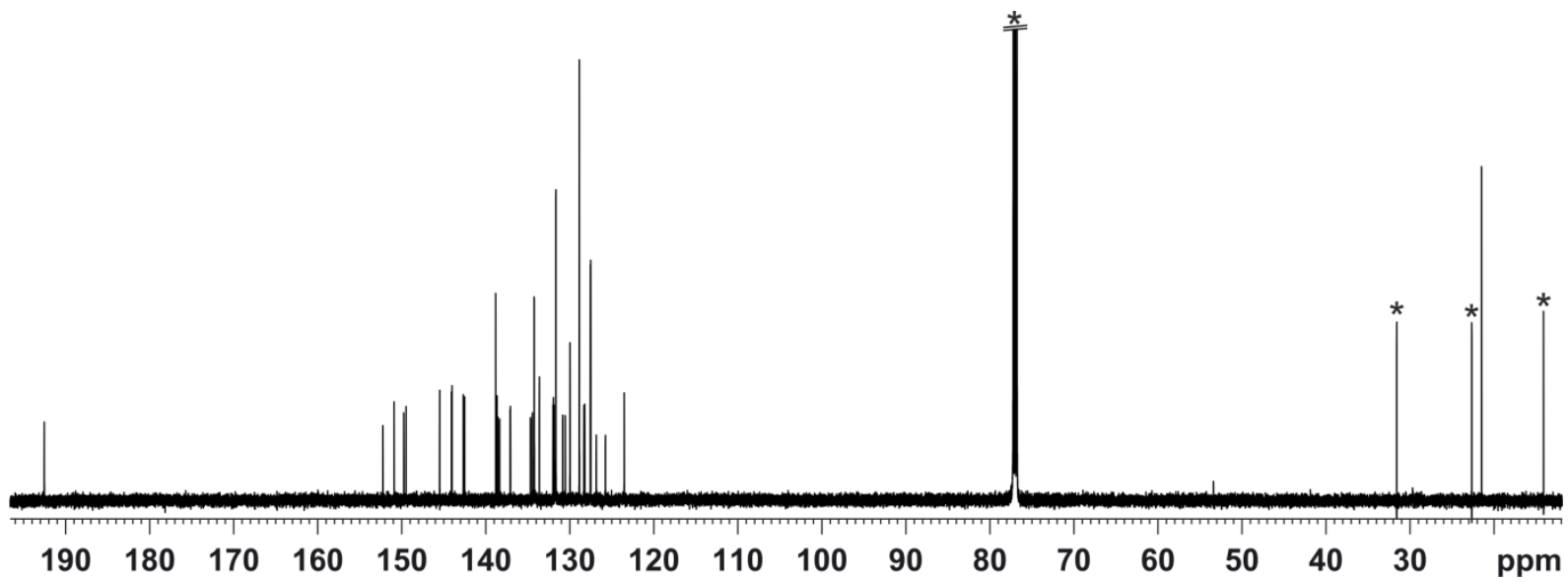

Figure S12. ${ }^{13} \mathrm{C}$ NMR for 2a-Pd(II) $\left(\mathrm{CDCl}_{3}, 151 \mathrm{MHz}, 300 \mathrm{~K}\right)$. 


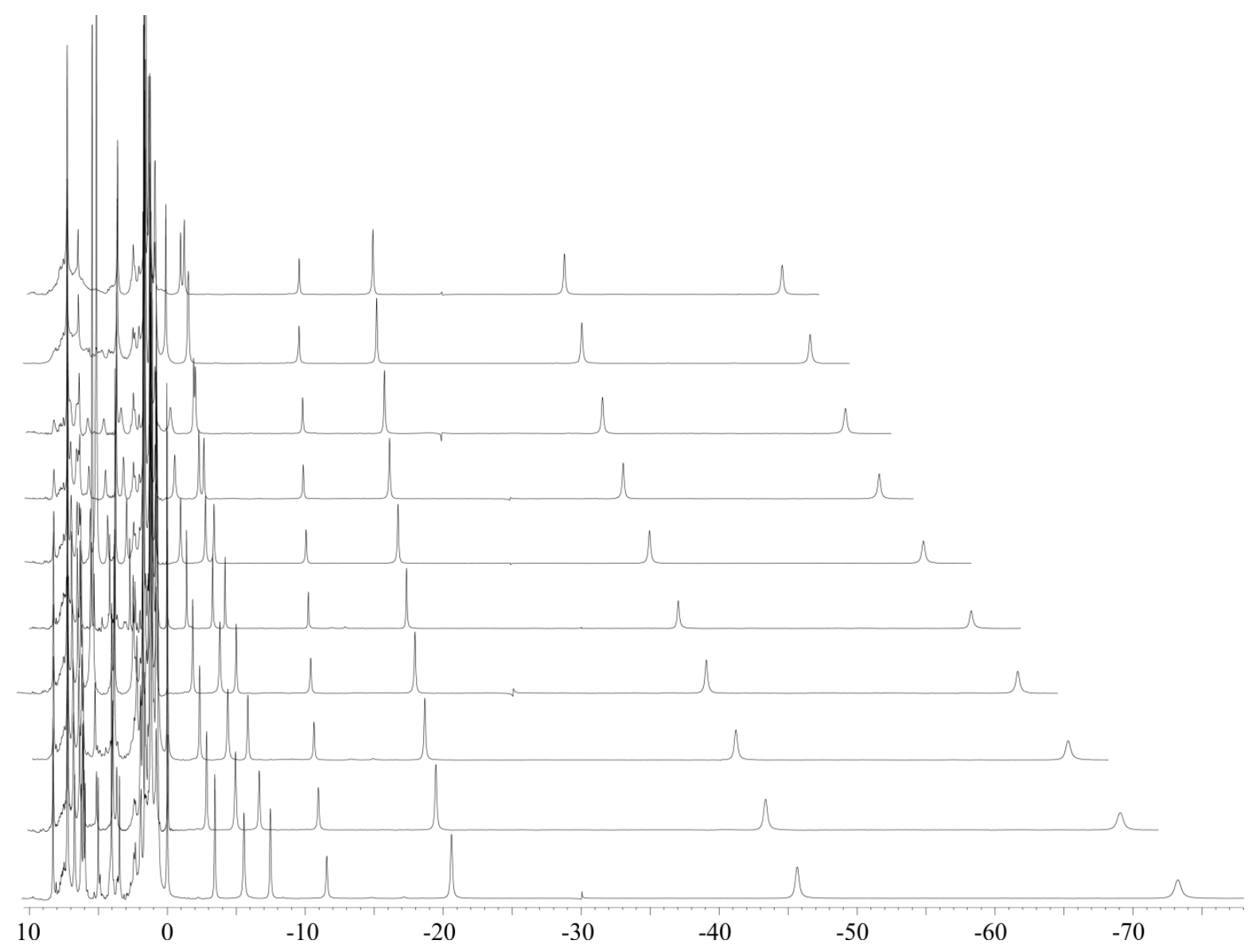

Figure S13. Variable-temperature ${ }^{1} \mathrm{H}$ NMR of paramagnetic complex 1-Ru(III) (600 MHz, $\mathrm{CDCl}_{3}$ ) from $300 \mathrm{~K}$ (top) to 210 $\mathrm{K}$ (bottom).

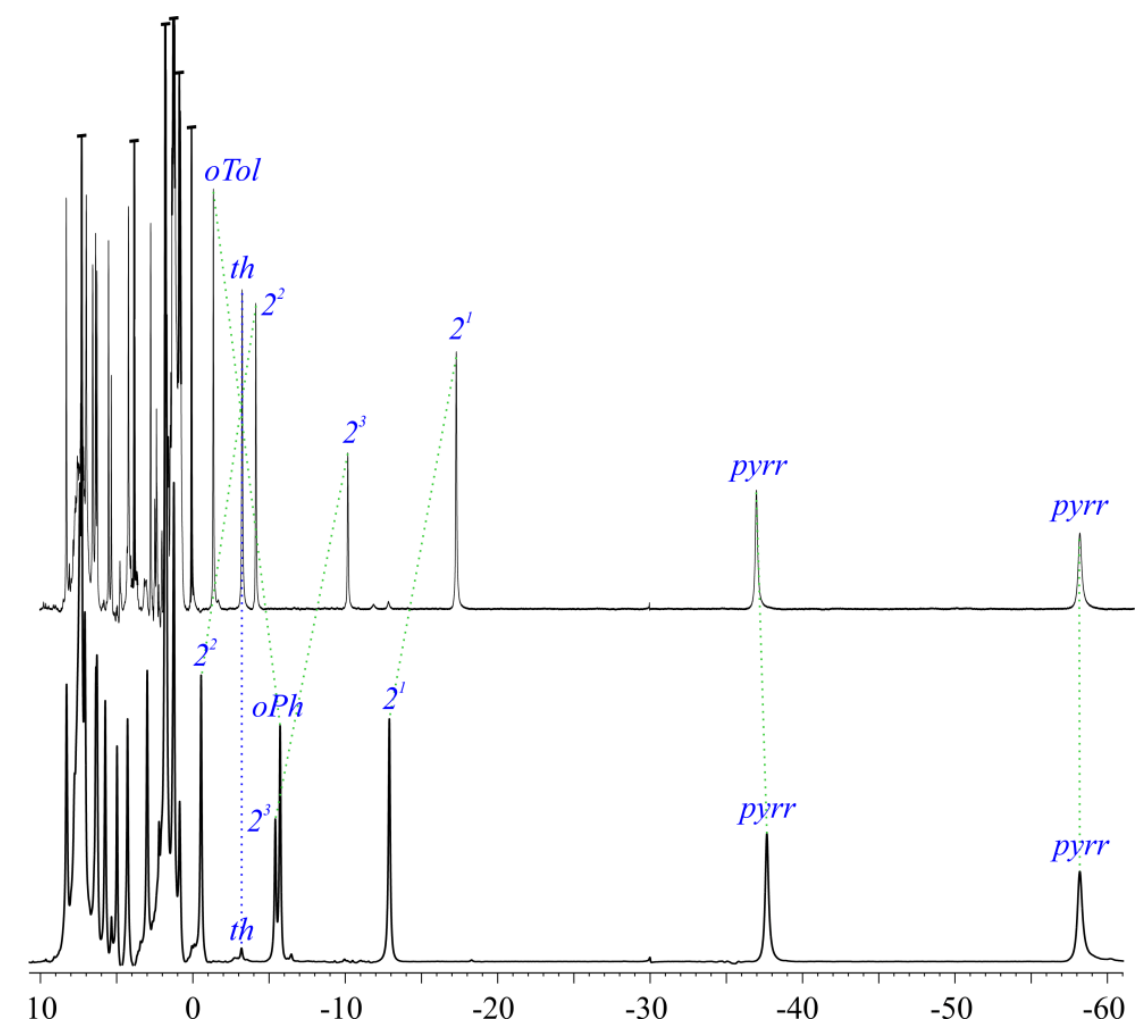

Figure S14. Comparison of ${ }^{1} \mathrm{H}$ NMR spectra $\left(600 \mathrm{MHz}, \mathrm{CDCl}_{3}, 250 \mathrm{~K}\right)$ of 1-Ru(III) and 1-Ru(III)- $d_{2}$ derivative. Respective signals connected with dotted lines (thiophene with blue one). Differences in spectra result from utilization of 5,10,15,20tetraphenyl ligand- $d_{2}$ instead of 5,20-diphenyl-10,15-ditolyl 1. 


\section{High resolution mass spectra}

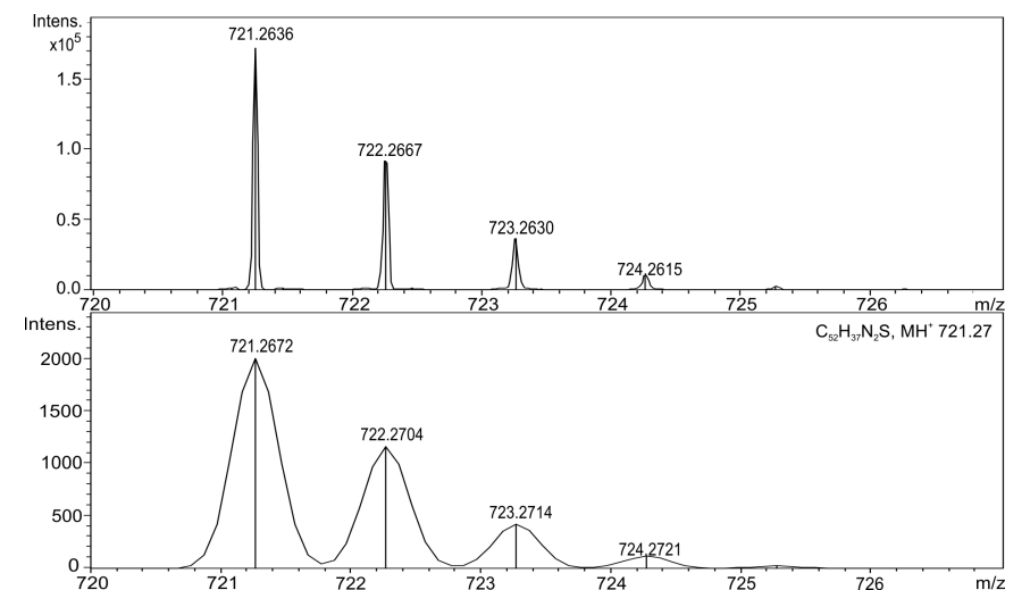

Figure S15. Experimental (upper) and simulated (lower) 1 HRMS spectra $\left(\mathrm{MH}^{+}\right)$.

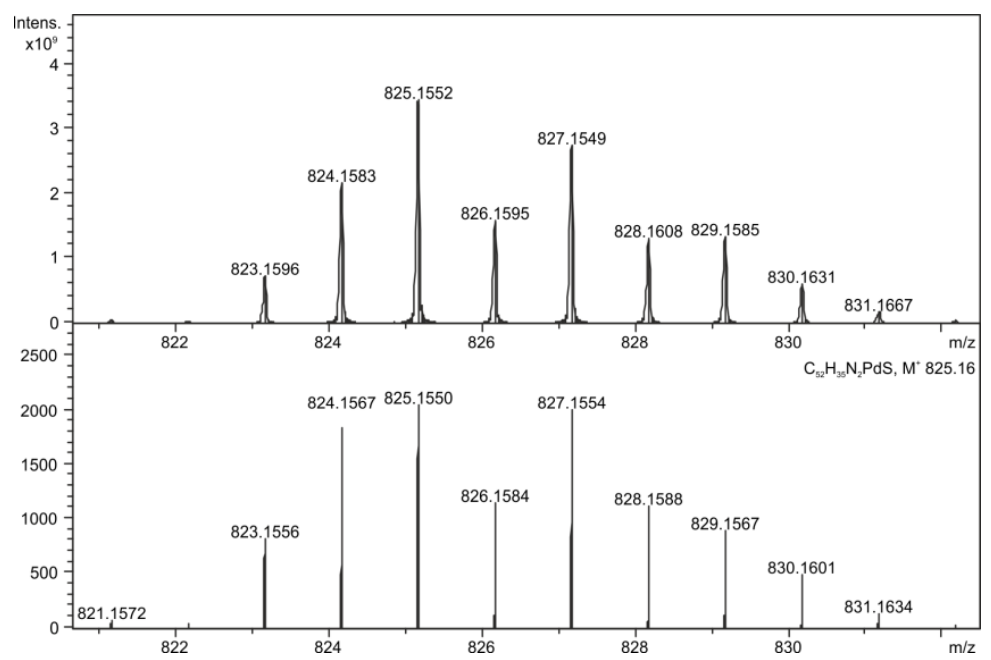

Figure S16. Experimental (upper) and simulated (lower) 1-Pd(II) $)^{+} \mathrm{HRMS}$ spectra $\left(\mathrm{M}^{+}\right)$.

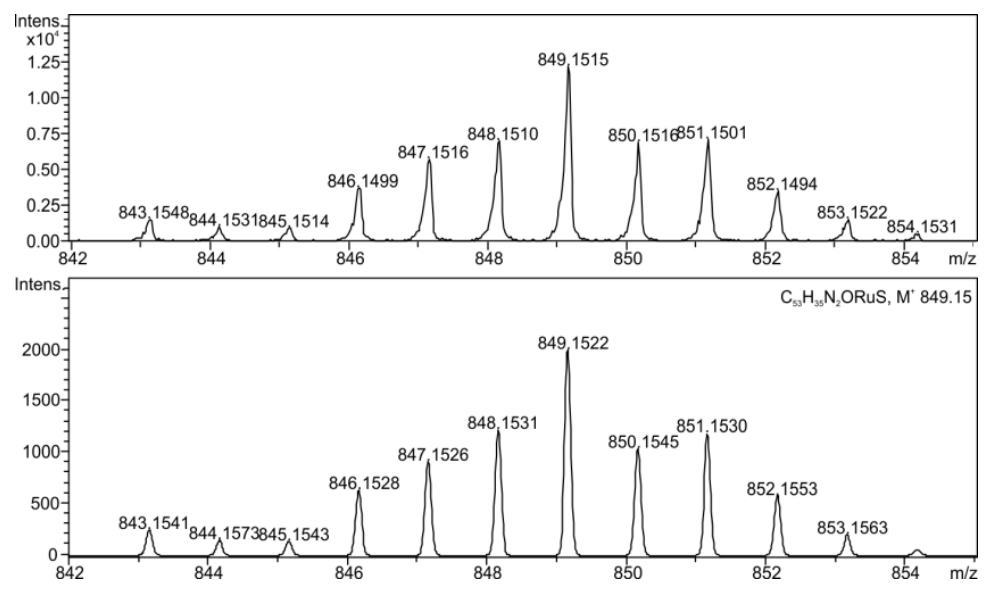

Figure S17. Experimental (upper) and simulated (lower) 1-Ru(II) HRMS spectra (M-Cl'). 


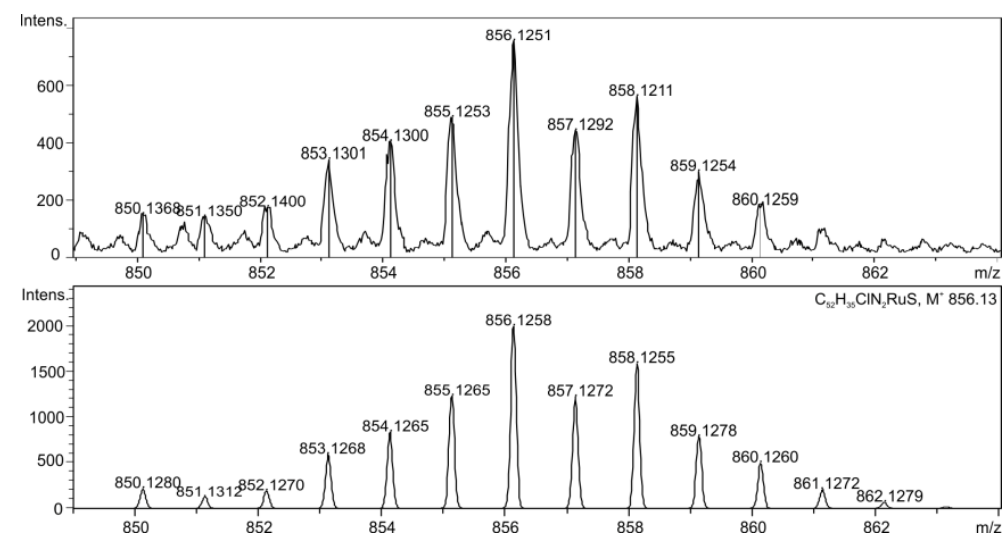

Figure S18. Experimental (upper) and simulated (lower) 1-Ru(III) HRMS spectra (M-Cl+).
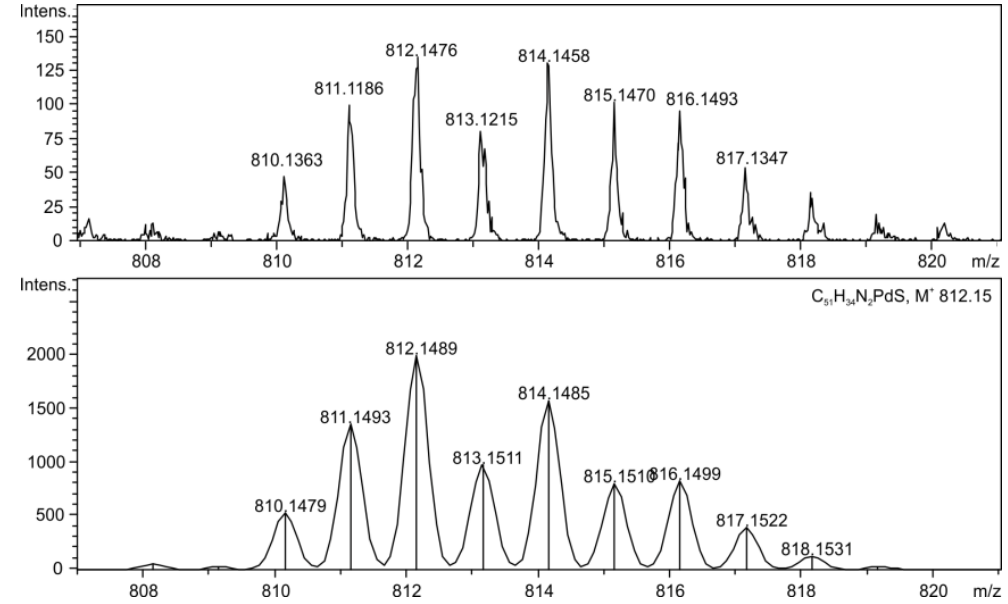

Figure S19. Experimental (upper) and simulated (lower) 2-Pd(II) HRMS spectra $\left(\mathrm{M}^{+}\right)$.

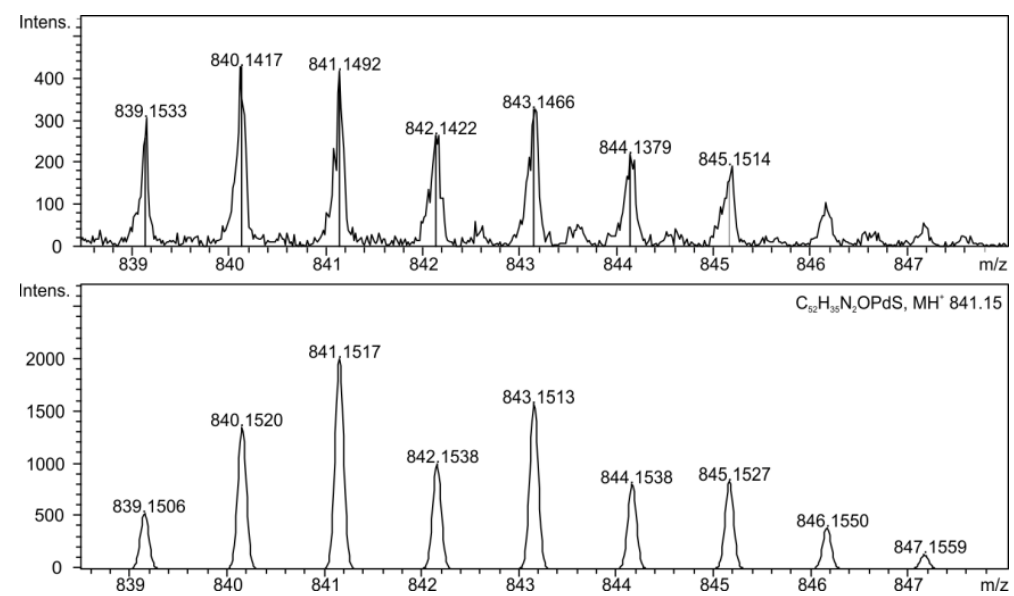

Figure S20. Experimental (upper) and simulated (lower) 2a-Pd(II) HRMS spectra $\left(\mathrm{MH}^{+}\right)$. 


\section{X-ray crystallography data}

Table S1.Crystal and structure refinement data for 1, 1-Ru(II), 1-Pd(II) ${ }^{+}$, and $\mathbf{2 a - P d ( I I ) ~}$

\begin{tabular}{|c|c|c|c|c|}
\hline symbol of the structure & $\begin{array}{l}\text { SAz } \\
{[1]}\end{array}$ & $\begin{array}{c}{[\mathbf{R u}(\mathrm{SAz}) \mathbf{C l}(\mathrm{CO})]} \\
{[1-\mathbf{R u}(\mathrm{II})]}\end{array}$ & $\begin{array}{c}{[\mathrm{Pd}(\mathrm{SAz})]_{2}\left[\mathrm{Pd}_{2} \mathbf{C l}_{6}\right]} \\
{\left[\mathbf{1 - P d}(\mathbf{I I})^{+}\right]}\end{array}$ & $\begin{array}{c}{[\mathrm{Pd}(\mathrm{SBzC}-\mathrm{CHO})]} \\
{[2 \mathrm{a}-\mathrm{Pd}(\mathrm{II})]}\end{array}$ \\
\hline empirical formula & $\mathrm{C}_{52} \mathrm{H}_{36} \mathrm{~N}_{2} \mathrm{~S}$ & $\mathrm{C}_{57} \mathrm{H}_{43} \mathrm{Cl}_{9} \mathrm{~N}_{2} \mathrm{ORuS}$ & $\mathrm{C}_{52} \mathrm{H}_{35} \mathrm{Cl}_{3} \mathrm{~N}_{2} \mathrm{O}_{0.90} \mathrm{Pd}_{2} \mathrm{~S}$ & $\mathrm{C}_{54.95} \mathrm{H}_{40.68} \mathrm{Cl}_{1.03} \mathrm{~N}_{2} \mathrm{OPdS}$ \\
\hline formula weight & 720.89 & 1224.11 & 1053.43 & 919.80 \\
\hline crystal size $\left[\mathrm{mm}^{3}\right]$ & $0.31 \times 0.06 \times 0.05$ & $0.20 \times 0.05 \times 0.02$ & $0.45 \times 0.12 \times 0.10$ & $0.36 \times 0.10 \times 0.07$ \\
\hline crystal system & monoclinic & monoclinic & monoclinic & triclinic \\
\hline space group & $C 2 / \mathrm{c}$ & $P 2_{1} / \mathrm{c}$ & $P 2_{1} / \mathrm{n}$ & $P \overline{1}$ \\
\hline $\mathbf{a}[\AA]$ & $45.775(7)$ & $10.995(2)$ & $13.086(3)$ & $10.635(3)$ \\
\hline $\mathbf{b}[\AA ̊]$ & $7.307(3)$ & $20.962(4)$ & $28.335(6)$ & $14.695(4)$ \\
\hline c [Å] & $27.470(5)$ & $23.410(8)$ & $16.858(4)$ & $15.144(5)$ \\
\hline$\alpha\left[^{\circ}\right]$ & 90 & 90 & 90 & $62.24(3)$ \\
\hline$\beta\left[^{\circ}\right]$ & $126.02(2)$ & $99.31(2)$ & $102.23(3)$ & $81.23(3)$ \\
\hline$\gamma\left[\left[^{\circ}\right]\right.$ & 90 & 90 & 90 & $89.46(4)$ \\
\hline $\mathbf{V}\left[\AA^{3}\right]$ & $7432(4)$ & $5324(2)$ & $6109(2)$ & 2064.7(12) \\
\hline $\mathbf{Z}$ & 8 & 4 & 4 & 2 \\
\hline density (calcd) $\left[\mathrm{Mg}^{\prime} \mathrm{m}^{-3}\right]$ & 1.289 & 1.527 & 1.145 & 1.479 \\
\hline$F(000)$ & 3024 & 2480 & 2109 & 943.4 \\
\hline coeff, $\mu\left[\mathrm{mm}^{-1}\right]$ & 0.128 & 0.828 & 0.784 & 0.612 \\
\hline abs. correction & none & none & analytical & analytical \\
\hline $\mathbf{T}_{\min / \max }$ & $\mathrm{n} / \mathrm{a}$ & $\mathrm{n} / \mathrm{a}$ & $0.876-0.938$ & $0.810-0.958$ \\
\hline $\mathbf{T}[\mathbf{K}]$ & $110.02(16)$ & $100.1(4)$ & $79.9(4)$ & $100(2)$ \\
\hline$\lambda[\AA]$ & 0.71073 & 0.71073 & 0.71073 & 0.71073 \\
\hline reflections coll., independ., obs. & $14761,6377,3633$ & $23673,10465,8552$ & $22967,11156,6001$ & $16434,7640,6035$ \\
\hline $\mathbf{R}_{\text {int }}$ & 0.0835 & 0.0241 & 0.0887 & 0.0466 \\
\hline data/parameters & $6377 / 498$ & $10465 / 658$ & $11156 / 552$ & $7640 / 608$ \\
\hline $\begin{array}{l}\text { final } R \text { indices } \\
(I>\mathbf{I}(\mathrm{I}))\end{array}$ & $\begin{array}{l}\mathrm{R}_{1}=0.0812 \\
\mathrm{wR}=0.1207\end{array}$ & $\begin{array}{l}\mathrm{R}_{1}=0.0390 \\
\mathrm{wR}=0.0528\end{array}$ & $\begin{array}{l}\mathrm{R}_{1}=0.0868 \\
\mathrm{wR}=0.1356\end{array}$ & $\begin{array}{l}\mathrm{R}_{1}=0.0498 \\
\mathrm{wR}=0.0696\end{array}$ \\
\hline $\begin{array}{l}\mathrm{R} \text { indices } \\
\text { (all data) }\end{array}$ & $\begin{array}{l}\mathrm{R}_{1}=0.1575 \\
\mathrm{wR}=0.1468\end{array}$ & $\begin{array}{l}\mathrm{R}_{1}=0.0848 \\
\mathrm{wR}=0.0918\end{array}$ & $\begin{array}{l}\mathrm{R}_{1}=0.2121 \\
\mathrm{wR}=0.2408\end{array}$ & $\begin{array}{l}\mathrm{R}_{1}=0.1039 \\
\mathrm{wR}=0.1140\end{array}$ \\
\hline GOF on $F^{2}$ & 1.062 & 1.018 & 0.943 & 1.051 \\
\hline Completness & 0.974 & 0.999 & 0.980 & 0.995 \\
\hline peak, hole $\left[e \cdot \AA^{-3}\right]$ & $0.30,-0.38$ & $1.09,-1.22$ & $1.07,-0.82$ & $0.81,-0.64$ \\
\hline CCDC numbers & 1437720 & 1437722 & 1437721 & 1437723 \\
\hline
\end{tabular}



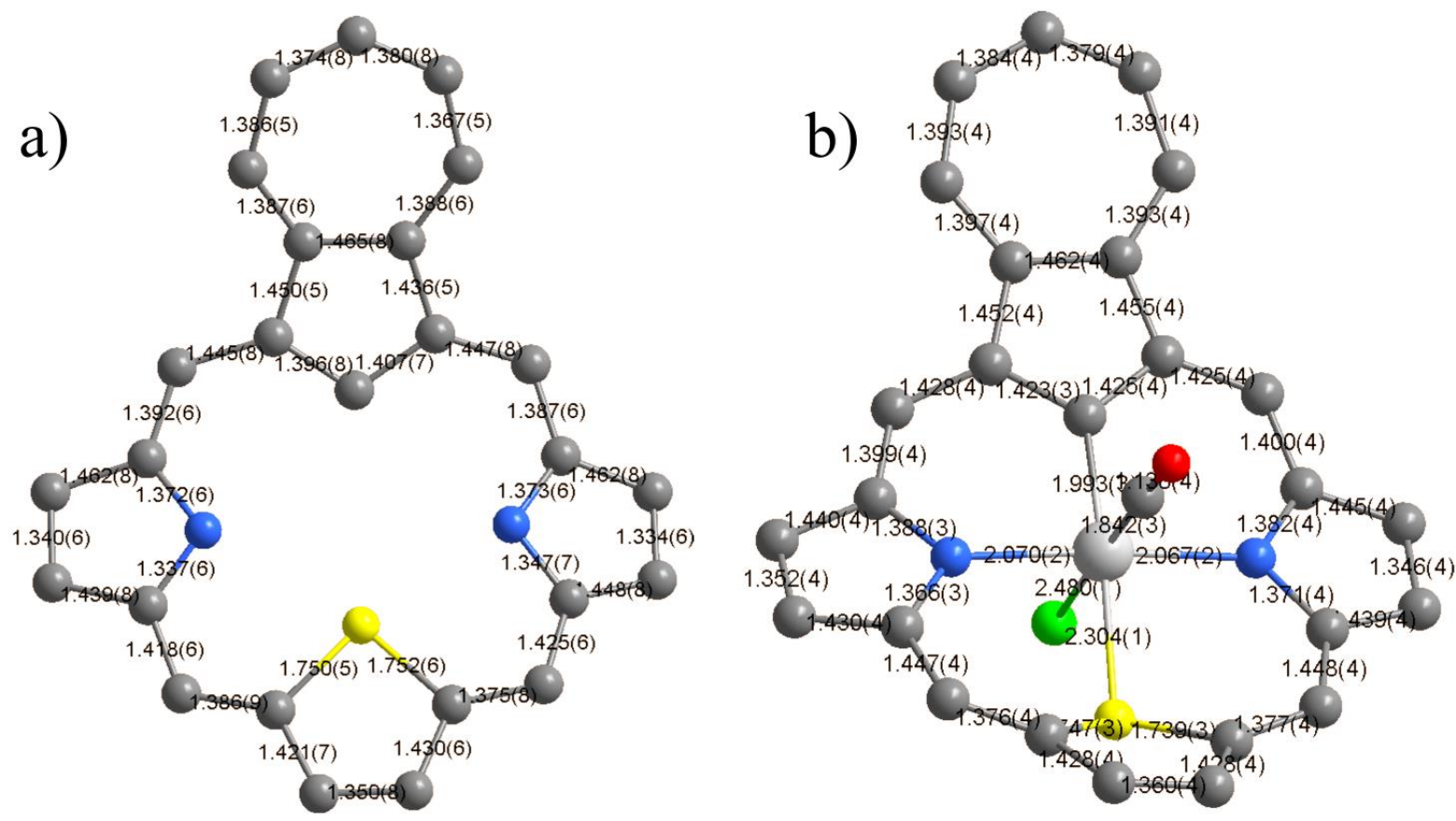

c)
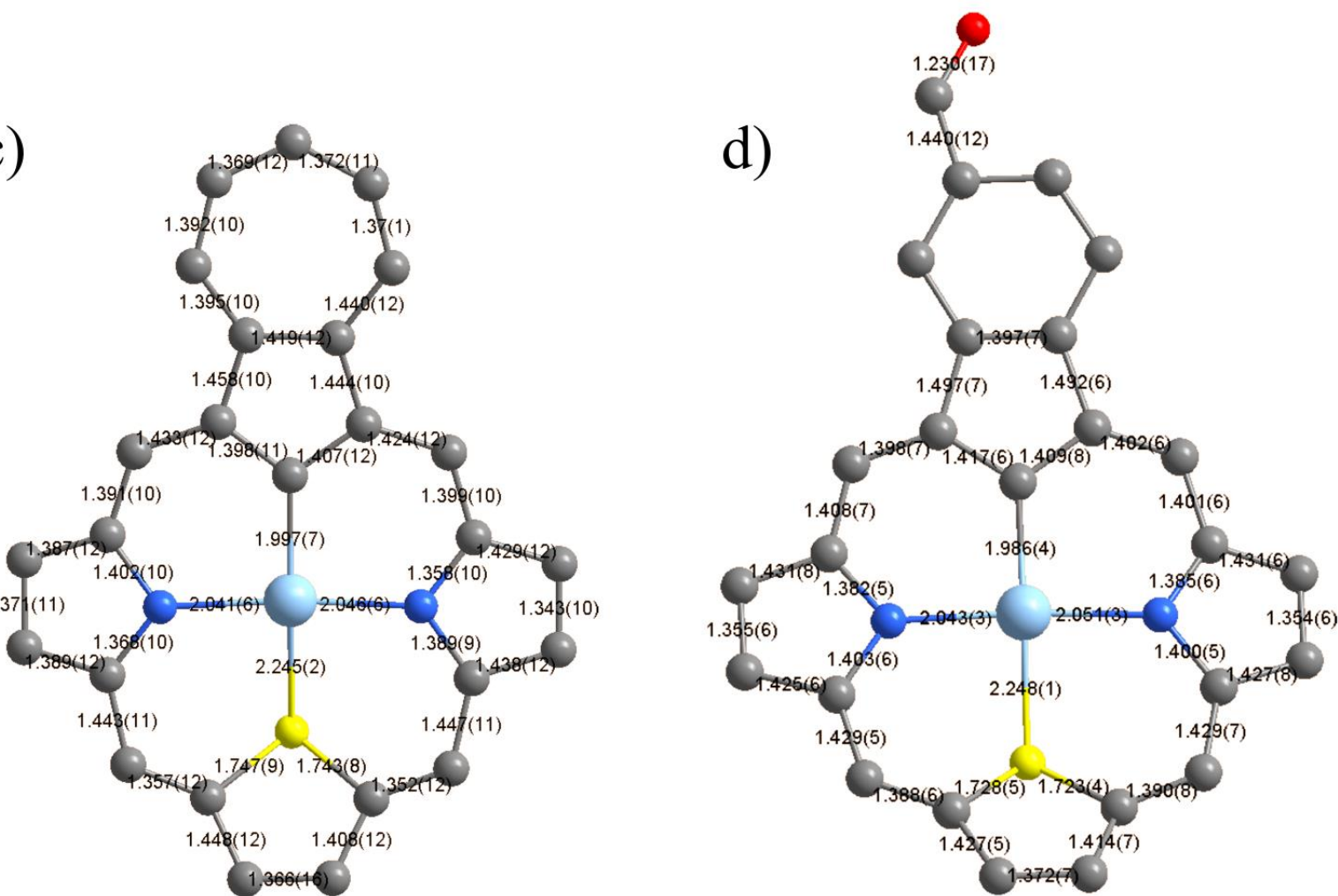

Figure S21. Selected bond distances $[\AA]$ for a) 1, b) 1-Ru(II), c) 1-Pd(II) ${ }^{+}$, and d) 2a-Pd(II). Hydrogens, meso rings, and solvent molecules (in the case of $1-\mathrm{Pd}(\mathrm{II})^{+}$also counterion) removed. 


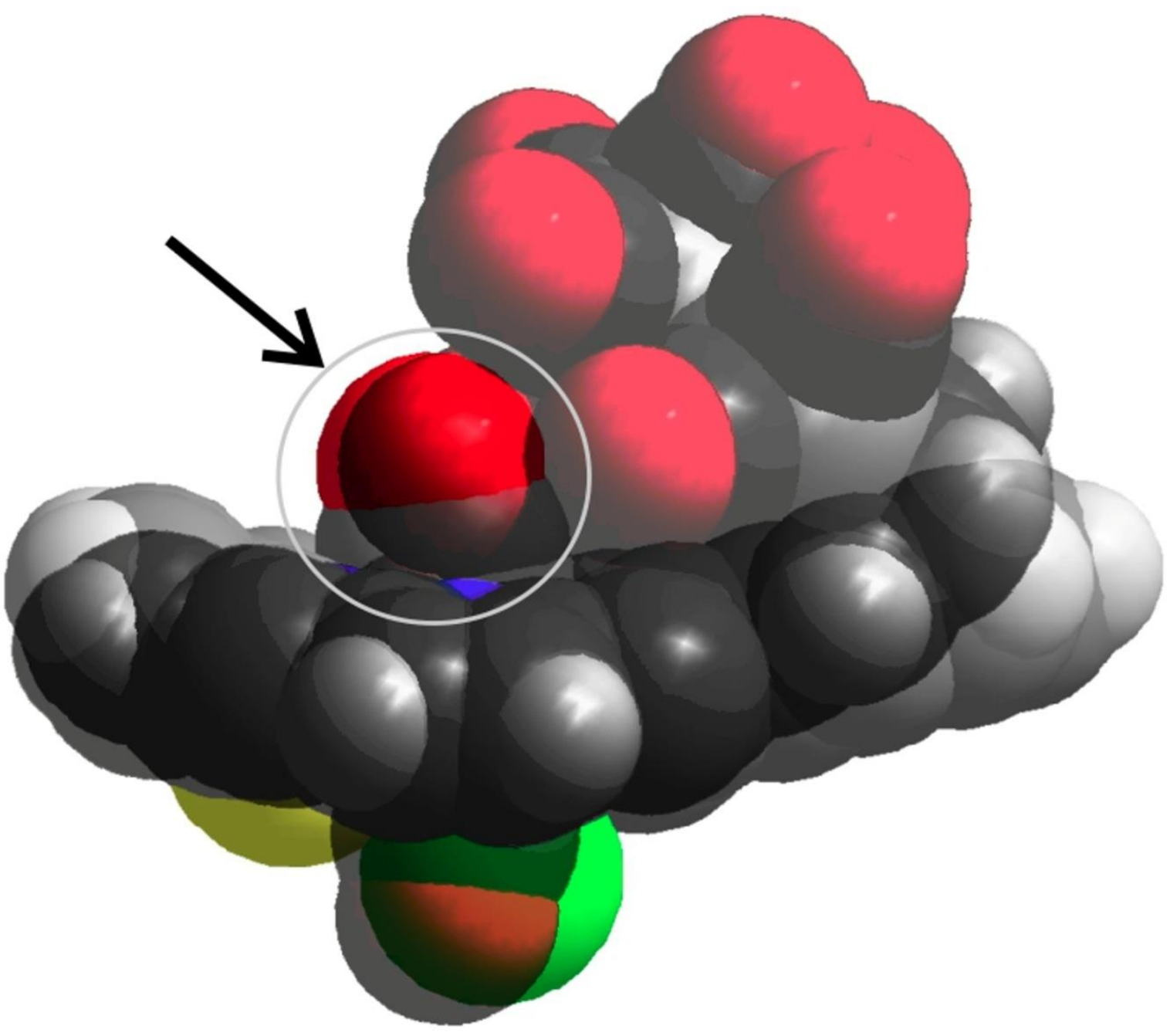

Figure S22. Imposed space filling structures of 1-Ru(II) and azuliporphyrin ruthenium cluster complex $\left[\mathrm{Ru}(\mathrm{TPAP})(\mathrm{CO})\left\{\mathrm{Ru}_{4}(\mathrm{CO})_{9}\right\}\right]$. One can see axial carbonyl ligand of $\mathbf{1}-\mathrm{Ru}(\mathrm{II})$ preventing formation of $\left\{\mathrm{Ru}_{4}(\mathrm{CO})_{9}\right\}$ cluster complex with 23-thiaazuliporphyrin. 


\section{DFT studies}
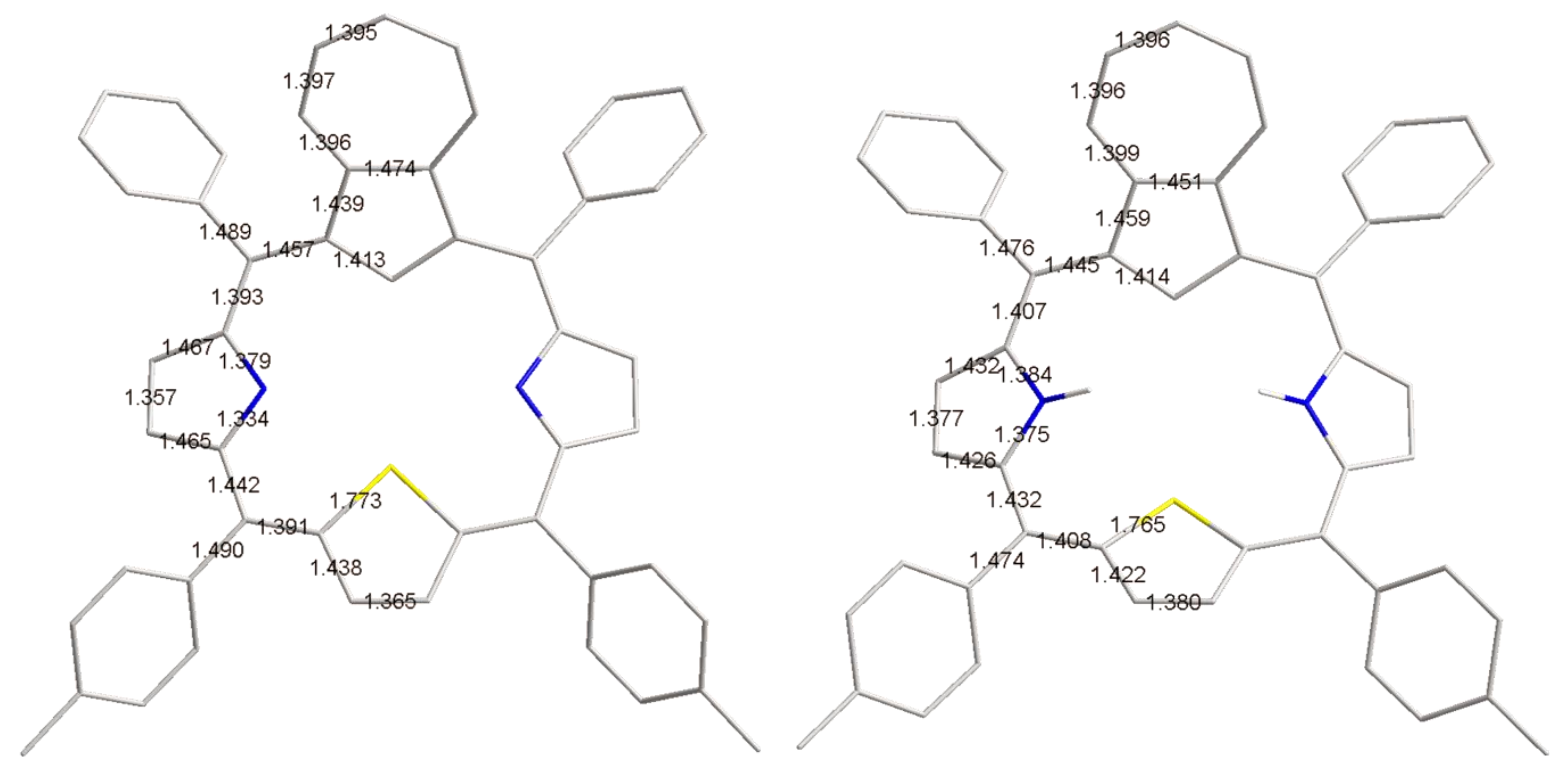

Figure S23. 1o (left) and 1- $\mathrm{H}_{2}{ }^{2+}{ }_{\mathrm{o}}$ (right) models [B3LYP/6-31(d,p)] with selected bond distances provided [ $\AA$ ]. One can see a higher degree of distances equalization for $1-\mathrm{H}_{2}{ }^{2+}$.

Table S2. Selected calculated [GIAO/BP86/6-31(d,p)] and experimental ${ }^{1} \mathrm{H}$ chemical shifts of $\mathbf{1}$ and $\mathbf{1}-\mathrm{H}_{2}{ }^{2+}$.

\begin{tabular}{ccccc}
\multicolumn{2}{c}{$\mathrm{SAz}(\mathbf{1})$} & \multicolumn{2}{c}{$\mathrm{SAzH}_{2}{ }^{2+}\left(\mathbf{1}-\mathbf{H}_{2}{ }^{2+}\right)$} \\
\hline Signal & Experimental & Calculated & Experimental & Calculated \\
$\mathbf{2}^{\mathbf{1}} \mathbf{3}^{\mathbf{1}}$ & 7.77 & 7.72 & 8.12 & 8.31 \\
$\mathbf{2}^{\mathbf{2}} \mathbf{3}^{\mathbf{2}}$ & 6.94 & 6.73 & 7.76 & 7.90 \\
$\mathbf{2}^{\mathbf{3}}$ & 7.30 & 7.12 & 7.96 & 8.26 \\
$\mathbf{2 1}$ & 4.04 & 3.13 & -0.78 & -1.11 \\
$\mathbf{7 , 1 8}$ & 7.84 & 7.88 & 8.38 & 8.59 \\
$\mathbf{8 , 1 7}$ & 7.28 & 7.40 & 8.06 & 8.38 \\
$\mathbf{1 2} \mathbf{1 3}$ & 8.08 & 8.32 & 9.12 & 9.43 \\
$\mathbf{C H}$ & 2.53 & 2.43 & 2.68 & 2.89 \\
\hline
\end{tabular}

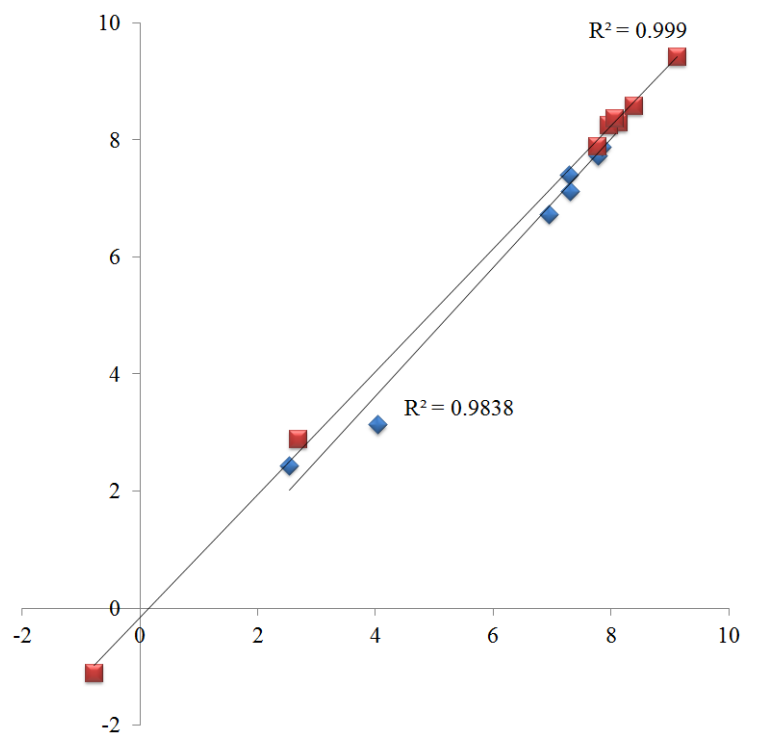

Figure S24. Correlation diagrams between calculated and experimental shifts (blue for 1, red for $\mathbf{1}-\mathrm{H}_{2}{ }^{2+}$ ). 


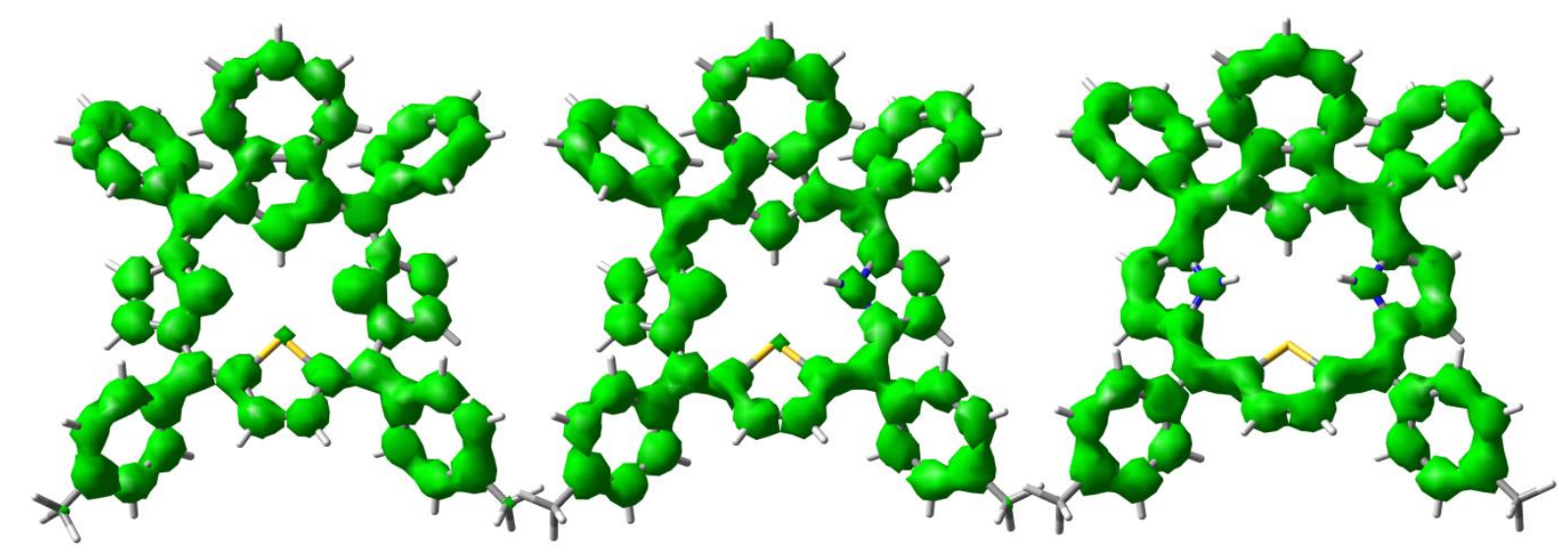

Figure S25. AICD plots [CSGT/B3LYP/6-31G(d,p)] (isoval. = 0.07 e/a.u. $\left.{ }^{3}\right)$ for $\mathbf{1}_{\mathrm{o}}(\mathrm{left}), \mathbf{1}-\mathrm{H}^{+}{ }_{\mathrm{o}}(\mathrm{center})$, and $\mathbf{1}-\mathrm{H}_{2}{ }^{2+}{ }_{\mathrm{o}}($ right $)$.

a)

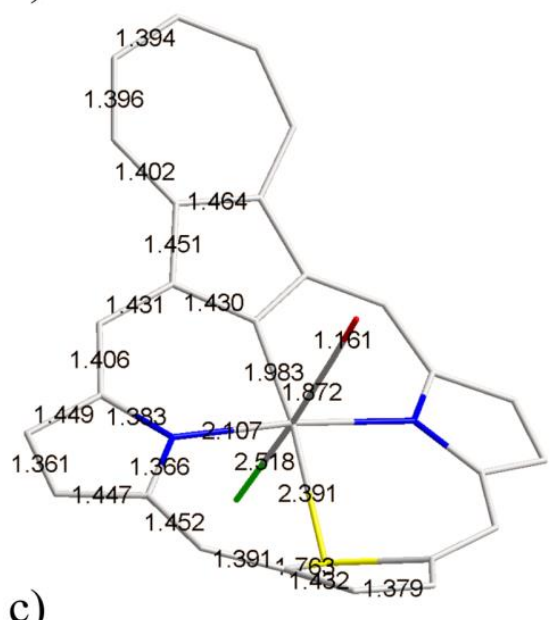

c)

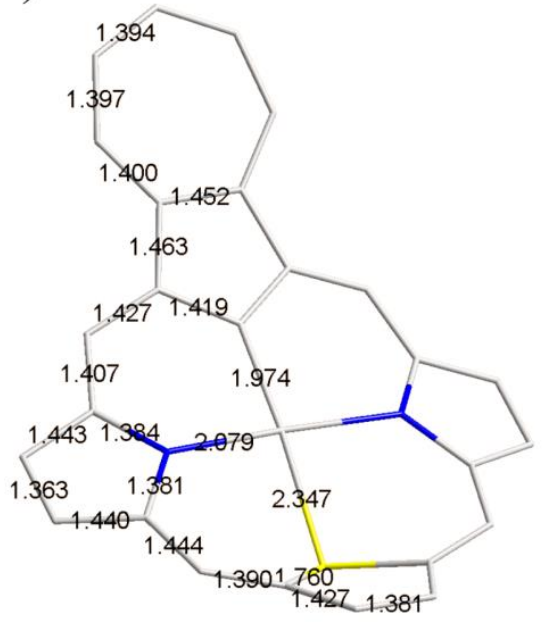

b)

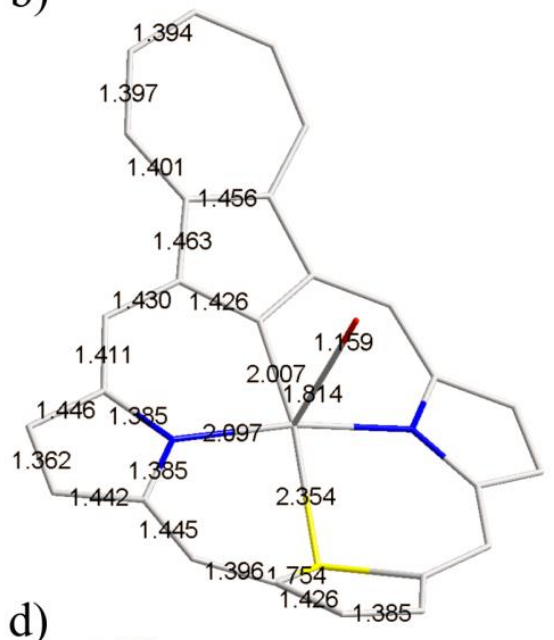

d)

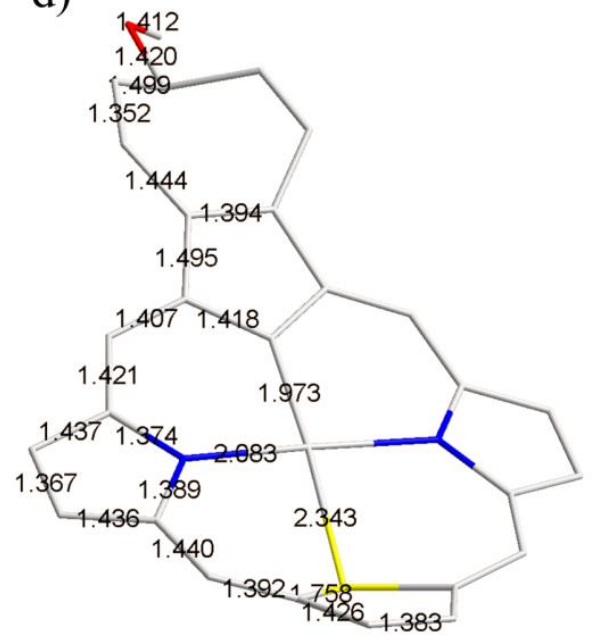

Figure S26. Selected bond distances $[\AA]$ for models (B3LYP/6-31(d,p)/LANL2DZ): a) 1-Ru(II) $)_{0}$, b) 1-Ru(II) ${ }^{+}$, c c) 1-Pd(II) ${ }^{+}{ }_{o}$, and d) 1-Pd(II)OMe . 

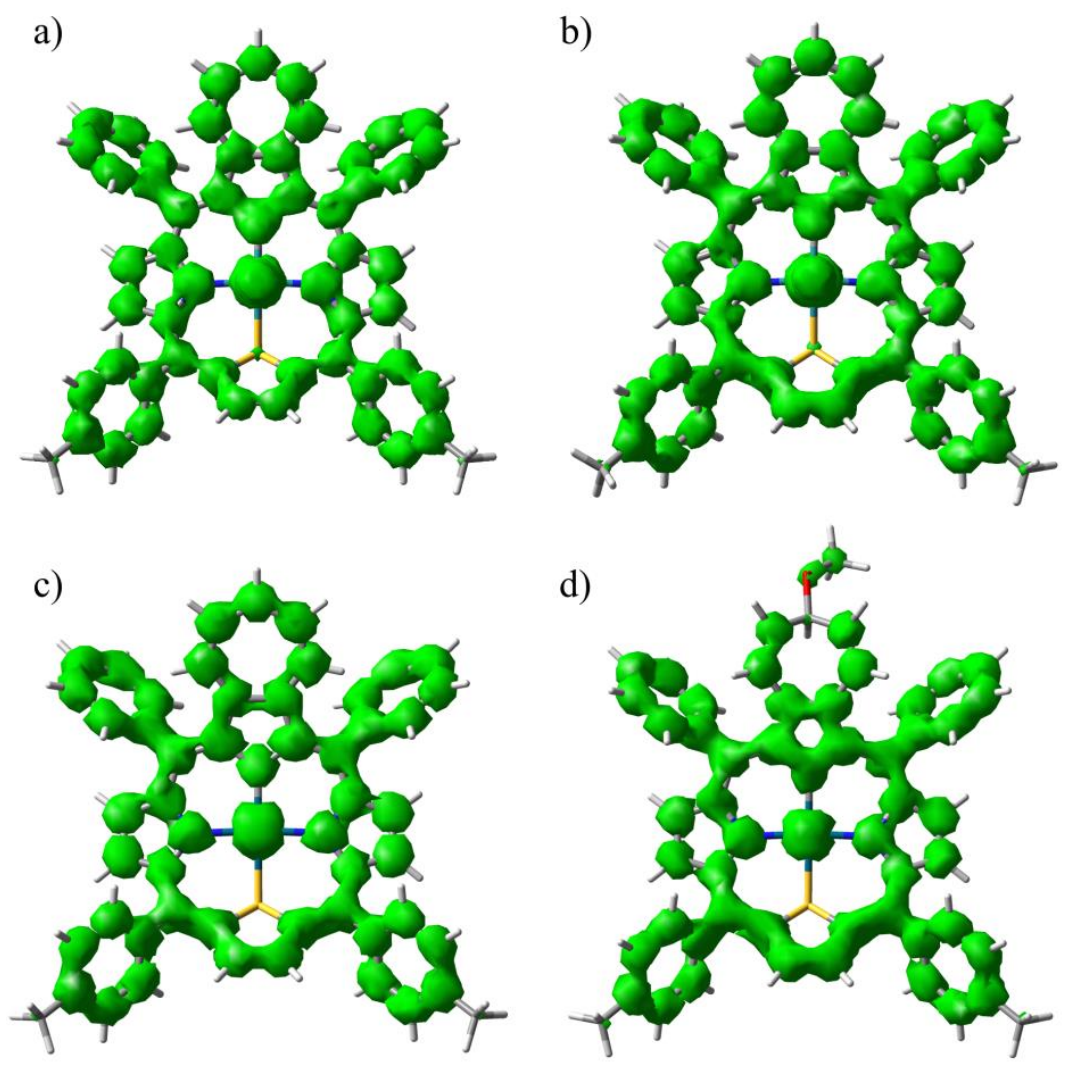

Figure S27. AICD plots (CSGT/BP86/6-31G(d,p)/LANL2DZ) (isoval. = 0.07 e/a.u. $\left.{ }^{3}\right)$ for: a) 1-Ru(II)o, b) 1-Ru(II) ${ }^{+}$, c c) 1$\mathrm{Pd}(\mathrm{II})^{+}$o, and d) 1-Pd(II)OMe.
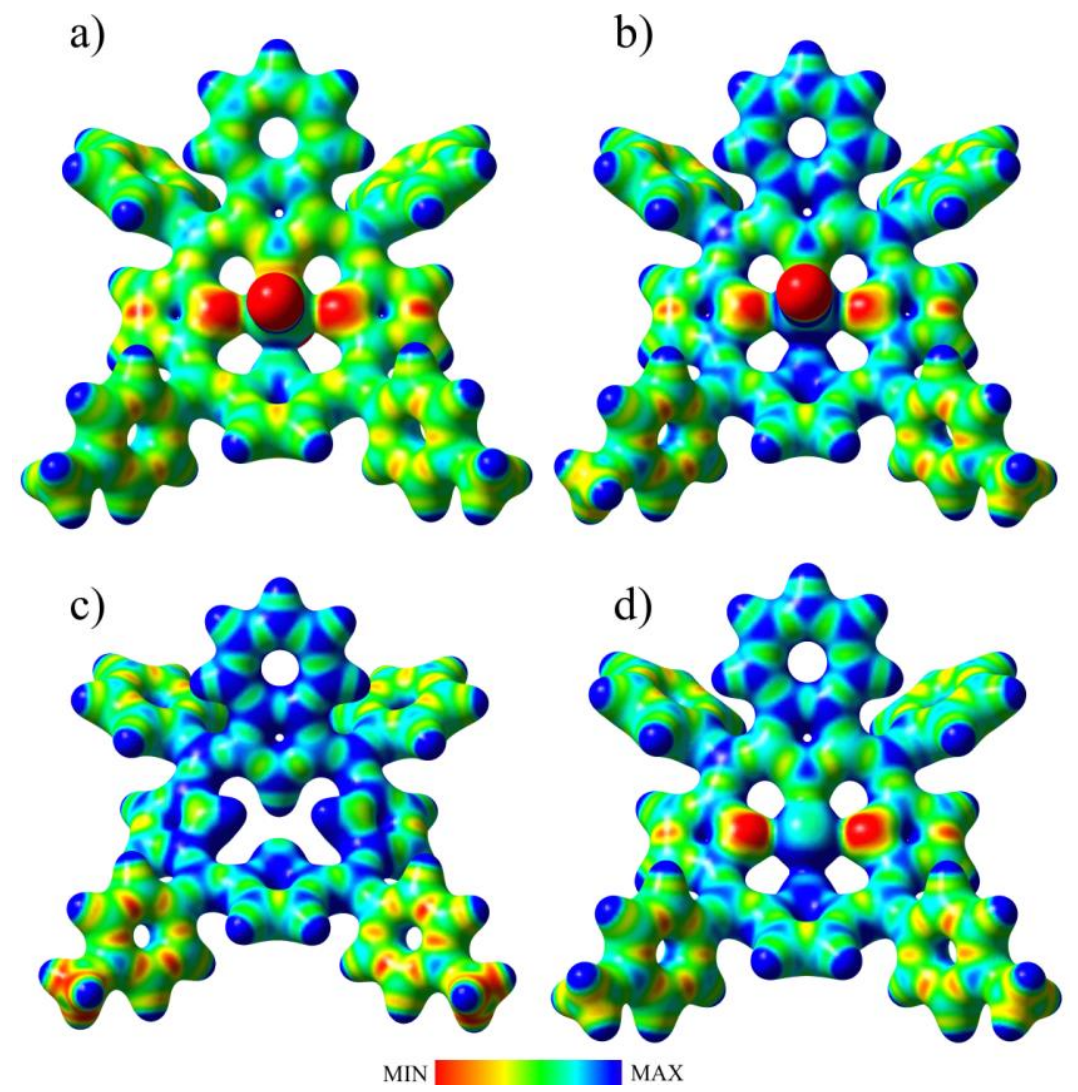

Figure S28. ESP maps (isoval. $=0.05$ e/a.u. $\left.{ }^{3}\right)$ for: a) $\mathbf{1}-\mathrm{Ru}(\mathrm{II})_{\mathrm{o}}(0.06-0.25$ a.u. $)$, b) $\mathbf{1}-\mathrm{Ru}(\mathrm{II})^{+}{ }_{\mathrm{o}}(0.15-0.30$ a.u. $)$, c) $1-\mathrm{H}_{2}{ }^{2+}{ }_{\mathrm{o}}$ (0.24-0.39 a.u.), and d) 1-Pd(II) ${ }^{+}$o $(0.15-0.30$ a.u.). 
Table S3. Hirshfeld partial atomic charges on heavy atoms (hydrogen atoms summed into heavy atoms) for 1-Ru(II) $)_{\mathrm{o}}$ and 1$\mathrm{Ru}(\mathrm{II})^{+}$. About 0.61 of unit charge is distributed on porphyrin complex scaffold $(0.39 \mathrm{e}$ is on meso substituents not shown in the table). Positive charge increase is among the highest on 7-membered ring atoms (highlighted).

\begin{tabular}{|c|c|c|c|}
\hline 1-Ru(II) $)_{\mathrm{o}}$ & 1-Ru(II) ${ }^{+}{ }_{0}$ & Difference & Heavy atom \\
\hline-0.061 & 0.002 & 0.063 & $\mathrm{Ru}$ \\
\hline-0.445 & & 0.445 & $\mathrm{Cl}$ \\
\hline-0.026 & -0.027 & 0.000 & 1 \\
\hline 0.027 & 0.032 & 0.005 & 2 \\
\hline 0.017 & 0.041 & 0.024 & $2^{1}$ \\
\hline 0.033 & 0.063 & 0.030 & $2^{2}$ \\
\hline 0.022 & 0.063 & 0.041 & $2^{3}$ \\
\hline 0.027 & 0.032 & 0.005 & 3 \\
\hline 0.017 & 0.041 & 0.024 & $3^{1}$ \\
\hline 0.033 & 0.063 & 0.030 & $3^{2}$ \\
\hline-0.026 & -0.027 & 0.000 & 4 \\
\hline 0.038 & 0.033 & -0.006 & 5 \\
\hline 0.043 & 0.038 & -0.004 & 6 \\
\hline-0.008 & 0.012 & 0.020 & 7 \\
\hline-0.017 & 0.004 & 0.020 & 8 \\
\hline 0.057 & 0.057 & 0.000 & 9 \\
\hline-0.001 & 0.008 & 0.009 & 10 \\
\hline-0.016 & -0.011 & 0.005 & 11 \\
\hline 0.013 & 0.037 & 0.024 & 12 \\
\hline 0.013 & 0.037 & 0.024 & 13 \\
\hline-0.016 & -0.011 & 0.005 & 14 \\
\hline-0.001 & 0.008 & 0.009 & 15 \\
\hline 0.057 & 0.057 & 0.000 & 16 \\
\hline-0.017 & 0.004 & 0.020 & 17 \\
\hline-0.008 & 0.012 & 0.020 & 18 \\
\hline 0.043 & 0.038 & -0.004 & 19 \\
\hline 0.038 & 0.033 & -0.006 & 20 \\
\hline 0.007 & -0.030 & -0.038 & 21 \\
\hline-0.105 & -0.122 & -0.017 & N22 \\
\hline 0.185 & 0.180 & -0.005 & $\mathrm{~S} 23$ \\
\hline-0.105 & -0.122 & -0.017 & $\mathrm{~N} 24$ \\
\hline-0.173 & -0.144 & 0.029 & $\mathrm{O}(\mathrm{CO})$ \\
\hline 0.176 & 0.216 & 0.040 & $\mathrm{C}(\mathrm{CO})$ \\
\hline
\end{tabular}



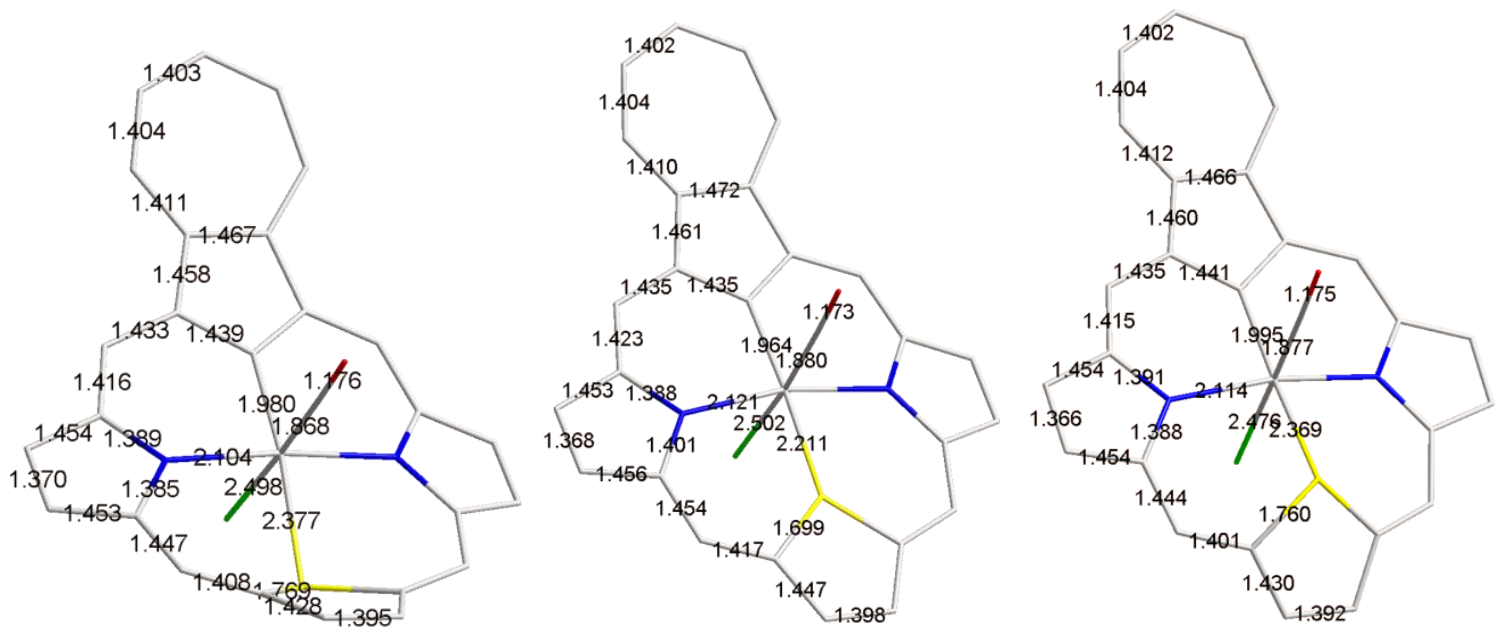

Figure S29. Selected bond distances $[\AA]$ for models (BP86/6-31(d,p)/LANL2DZ): 1-Ru(II)- $1_{o}$ (left) 1-Ru(II)- $t_{o}$ (center), and 1-Ru(II)-2o (right).

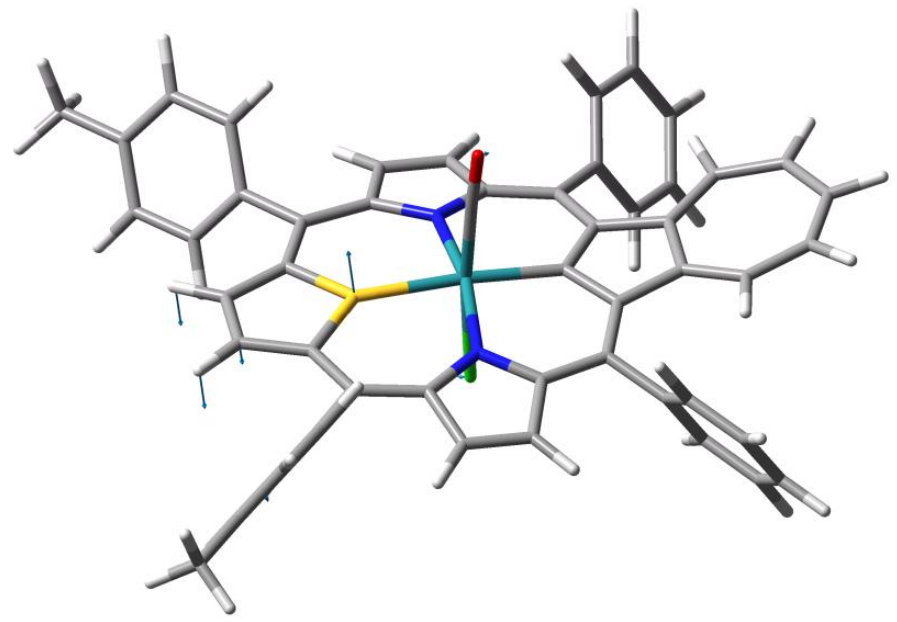

Figure S30. 1-Ru(II)- $t_{o}$ model showing one imaginary frequency vibration. Arrows point directions and relative amplitude of atoms movement.

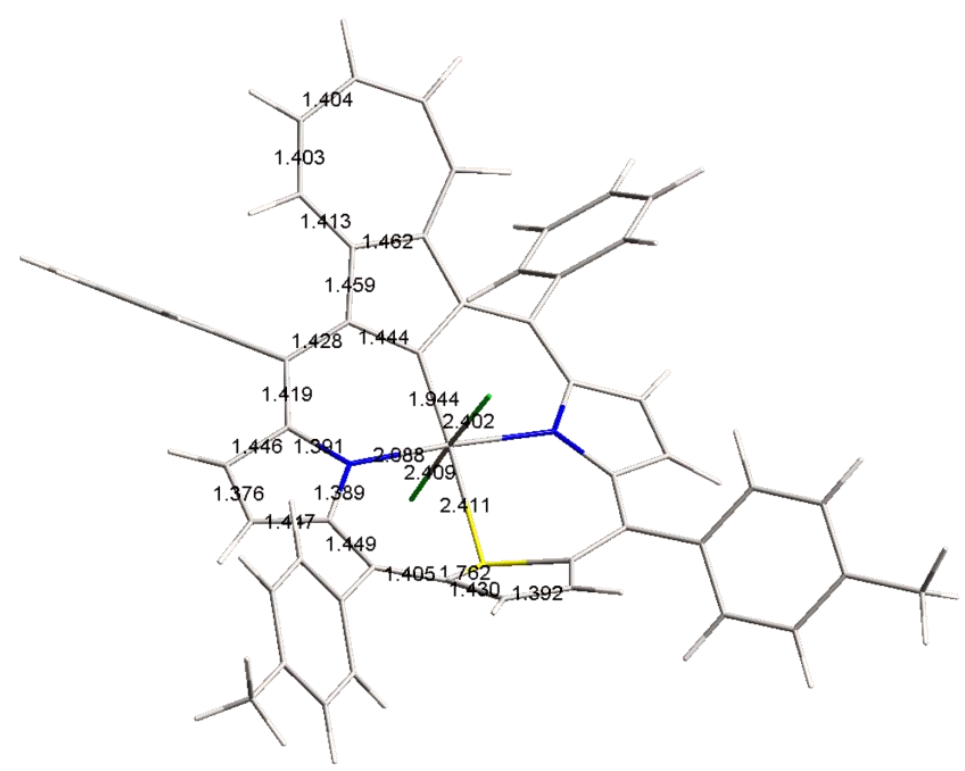

Figure S31. Selected bond distances $[\AA]$ for 1-Ru(III) $)_{\text {o }}$ (uBP86/6-31(d,p)/LANL2DZ): These geometry was used to calculate molecular orbital geometries and energies. 

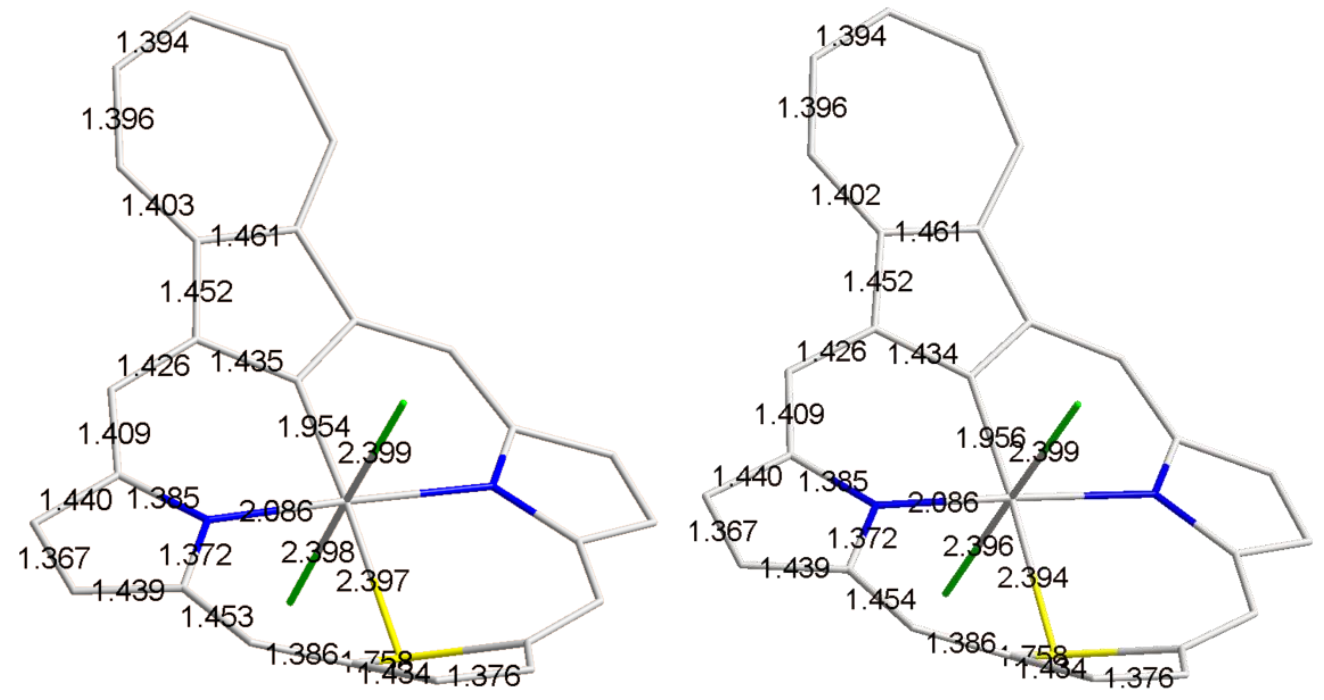

Figure S32. Selected bond distances $[\AA]$ for models (uB3LYP/6-31(d,p)/SDD): 1-Ru(III) $)_{0}-$ EGS (left) and 1-Ru(III) $)_{o}-$ EES (right). When LANL2DZ pseudopotential was used the states were switched meaning that very subtle geometrical changes determine the electronic ground state. These geometries were used to calculate spin density distribution.

Table S4. Partial atomic spin densities on heavy atoms (hydrogen atoms summed into heavy atoms) for $1-\mathrm{Ru}(\mathrm{III})_{\mathrm{o}}$ in electronic ground (EGS) and excited state (EES). The last column contains mixture of two states with arbitrarily chosen populations for best experimental fit.

\begin{tabular}{|c|c|c|c|}
\hline Heavy atom & EGS & EES & $\begin{array}{c}\text { 0.855 EGS + } \\
\text { 0.145·EES }\end{array}$ \\
\hline $2^{1}, 3^{1}$ & -0.0098 & 0.0654 & 0.0011 \\
\hline $2^{2}, 3^{2}$ & 0.0079 & -0.0449 & 0.0002 \\
\hline $2^{3}$ & -0.0108 & 0.0691 & 0.0008 \\
\hline Pyrr 7, 18 & 0.0138 & -0.0089 & 0.0105 \\
\hline Pyrr 8, 17 & 0.0111 & 0.0139 & 0.0115 \\
\hline Th 12,13 & 0.0004 & 0.0041 & 0.0009 \\
\hline $5 o \mathrm{Ph}$ & -0.0005 & -0.0019 & -0.0007 \\
\hline $5 o \mathrm{Ph}^{\prime}$ & -0.0005 & -0.0021 & -0.0008 \\
\hline $5 \mathrm{mPh}$ & 0.0001 & 0.0008 & 0.0002 \\
\hline $5 \mathrm{mPh}$ & 0.0001 & 0.0007 & 0.0002 \\
\hline $5 p \mathrm{Ph}$ & -0.0002 & -0.0015 & -0.0004 \\
\hline $10 \mathrm{oTol}$ & -0.0002 & 0.0012 & 0.0000 \\
\hline 10 oTol' & -0.0002 & 0.0013 & 0.0000 \\
\hline $10 m$ Tol & -0.0001 & -0.0006 & -0.0001 \\
\hline 10 mTol' & 0.0000 & -0.0004 & 0.0000 \\
\hline $\mathrm{CH}_{3}$ & 0.0000 & 0.0000 & 0.0000 \\
\hline $\mathrm{Cl}$ & 0.0537 & 0.0922 & 0.0593 \\
\hline $\mathrm{Cl}^{\prime}$ & 0.0683 & 0.0696 & 0.0685 \\
\hline $\mathrm{Ru}$ & 0.8746 & 0.7939 & 0.8629 \\
\hline
\end{tabular}




\section{DFT Coordinates}

1。 [B3LYP/6-31G(d,p)]

\begin{tabular}{|c|c|c|c|}
\hline $\mathrm{N}$ & -0.18529900 & -2.28890300 & 0.01485500 \\
\hline $\mathrm{C}$ & -1.24975000 & -3.07423500 & -0.15970400 \\
\hline $\mathrm{C}$ & 0.91906200 & -3.02247700 & -0.36488800 \\
\hline $\mathrm{N}$ & -0.18522500 & 2.28897500 & 0.01503000 \\
\hline $\mathrm{C}$ & -1.24963400 & 3.07438000 & -0.15947800 \\
\hline $\mathrm{C}$ & 0.91919500 & 3.02258300 & -0.36447900 \\
\hline $\mathrm{S}$ & -1.65144900 & 0.00005200 & 0.15855500 \\
\hline $\mathrm{C}$ & -2.88400500 & 1.27037200 & 0.25352000 \\
\hline $\mathrm{C}$ & -2.88406400 & -1.27022200 & 0.25354900 \\
\hline $\mathrm{C}$ & 2.55297400 & 1.16650900 & 0.06066700 \\
\hline $\mathrm{C}$ & 1.86086600 & 0.00003200 & -0.33428500 \\
\hline $\mathrm{C}$ & 2.22496700 & 2.54596300 & -0.27559800 \\
\hline $\mathrm{C}$ & 3.68703200 & 0.73696500 & 0.83620800 \\
\hline $\mathrm{C}$ & 4.54748800 & 1.57302400 & 1.54934300 \\
\hline $\mathrm{C}$ & 3.68701400 & -0.73716900 & 0.83606400 \\
\hline $\mathrm{C}$ & 5.63223800 & 1.25493000 & 2.37079900 \\
\hline $\mathrm{H}$ & 4.34701100 & 2.63426400 & 1.45691100 \\
\hline $\mathrm{C}$ & 6.12184900 & -0.00034800 & 2.73080000 \\
\hline $\mathrm{H}$ & 6.15604600 & 2.10710900 & 2.79677600 \\
\hline $\mathrm{C}$ & 5.63223400 & -1.25551900 & 2.37050300 \\
\hline $\mathrm{H}$ & 6.98558400 & -0.00042500 & 3.39228400 \\
\hline $\mathrm{C}$ & 4.54745300 & -1.57340300 & 1.54897800 \\
\hline $\mathrm{C}$ & 2.55291000 & -1.16652600 & 0.06045700 \\
\hline $\mathrm{H}$ & 4.34697400 & -2.63462200 & 1.45628800 \\
\hline $\mathrm{H}$ & 6.15603400 & -2.10781500 & 2.79625600 \\
\hline $\mathrm{C}$ & 2.22485500 & -2.54591500 & -0.27601900 \\
\hline $\mathrm{C}$ & 3.34676300 & -3.47306500 & -0.58943900 \\
\hline $\mathrm{C}$ & 4.36602400 & -3.07175600 & -1.47369000 \\
\hline $\mathrm{C}$ & 3.43223200 & -4.75816800 & -0.02037400 \\
\hline $\mathrm{C}$ & 5.41710600 & -3.92804700 & -1.79267300 \\
\hline $\mathrm{H}$ & 4.31485600 & -2.08461300 & -1.92200500 \\
\hline $\mathrm{C}$ & 5.48514300 & -5.20155900 & -1.22286400 \\
\hline $\mathrm{H}$ & 6.18425500 & -3.60171100 & -2.48903200 \\
\hline $\mathrm{C}$ & 4.49060100 & -5.61088800 & -0.33313100 \\
\hline $\mathrm{H}$ & 6.30835000 & -5.86667100 & -1.46658000 \\
\hline $\mathrm{H}$ & 4.54102500 & -6.59431100 & 0.12552700 \\
\hline $\mathrm{H}$ & 2.67373800 & -5.07251000 & 0.68880200 \\
\hline $\mathrm{C}$ & 0.48968600 & -4.34449800 & -0.83522900 \\
\hline $\mathrm{C}$ & -0.85928200 & -4.38299400 & -0.69060300 \\
\hline $\mathrm{H}$ & 1.12590500 & -5.11356500 & -1.24797400 \\
\hline $\mathrm{H}$ & -1.52965200 & -5.19062900 & -0.94948000 \\
\hline $\mathrm{C}$ & -2.59709000 & -2.61955400 & 0.07773900 \\
\hline $\mathrm{C}$ & -3.69071600 & -3.63140900 & 0.08677800 \\
\hline $\mathrm{C}$ & -4.79691500 & -3.53333900 & -0.77333200 \\
\hline $\mathrm{C}$ & -3.63229600 & -4.73890400 & 0.95030000 \\
\hline $\mathrm{C}$ & -4.64388200 & -5.69613600 & 0.96080500 \\
\hline $\mathrm{H}$ & -2.78716100 & -4.83980100 & 1.62453700 \\
\hline $\mathrm{C}$ & -5.75293200 & -5.59092000 & 0.11070700 \\
\hline $\mathrm{H}$ & -4.57270300 & -6.53910200 & 1.64412400 \\
\hline $\mathrm{C}$ & -5.80612400 & -4.49456700 & -0.75885800 \\
\hline $\mathrm{C}$ & -6.86285700 & -6.61418600 & 0.14748500 \\
\hline $\mathrm{H}$ & -6.64490600 & -4.39661300 & -1.44395900 \\
\hline $\mathrm{H}$ & -4.85098400 & -2.70666400 & -1.47494500 \\
\hline
\end{tabular}




$\begin{array}{lrrr}\mathrm{C} & -4.17245000 & -0.68241300 & 0.50310600 \\ \mathrm{C} & -4.17242000 & 0.68263600 & 0.50307400 \\ \mathrm{H} & -5.05695800 & -1.28518000 & 0.66644700 \\ \mathrm{H} & -5.05690300 & 1.28545600 & 0.66637400 \\ \mathrm{C} & -2.59699400 & 2.61969800 & 0.07778900 \\ \mathrm{C} & -3.69066600 & 3.63151500 & 0.08664500 \\ \mathrm{C} & -3.63272600 & 4.73878000 & 0.95032700 \\ \mathrm{C} & -4.79641900 & 3.53360200 & -0.77420500 \\ \mathrm{C} & -5.80552700 & 4.49479500 & -0.76023400 \\ \mathrm{H} & -4.84998300 & 2.70707100 & -1.47603300 \\ \mathrm{C} & -5.75287200 & 5.59101200 & 0.10970900 \\ \mathrm{H} & -6.64378600 & 4.39709200 & -1.44602400 \\ \mathrm{C} & -4.64439500 & 5.69607500 & 0.96038700 \\ \mathrm{C} & -6.86354800 & 6.61346300 & 0.14618200 \\ \mathrm{H} & -4.57362200 & 6.53897800 & 1.64380100 \\ \mathrm{H} & -2.78791200 & 4.83967200 & 1.62496700 \\ \mathrm{C} & -0.85909000 & 4.38321300 & -0.69011800 \\ \mathrm{C} & 0.48991200 & 4.34476400 & -0.83445500 \\ \mathrm{H} & -1.52940800 & 5.19091700 & -0.94891500 \\ \mathrm{H} & 1.12619900 & 5.11393300 & -1.24690600 \\ \mathrm{C} & 3.34691600 & 3.47314600 & -0.58878900 \\ \mathrm{C} & 3.43251700 & 4.75805200 & -0.01930200 \\ \mathrm{C} & 4.36608900 & 3.07205600 & -1.47324200 \\ \mathrm{C} & 4.49091600 & 5.61080800 & -0.33186500 \\ \mathrm{H} & 2.67411200 & 5.07220100 & 0.69005400 \\ \mathrm{C} & 5.48536000 & 5.20170900 & -1.22181100 \\ \mathrm{H} & 4.54144300 & 6.59407300 & 0.12712100 \\ \mathrm{C} & 5.41720000 & 3.92838600 & -1.79202800 \\ \mathrm{H} & 6.30859300 & 5.86684700 & -1.46537100 \\ \mathrm{H} & 6.18427400 & 3.60222900 & -2.48855400 \\ \mathrm{H} & 4.31482500 & 2.08506400 & -1.92187800 \\ \mathrm{H} & 0.94957300 & 0.00011500 & -0.91245300 \\ \mathrm{H} & -7.38785500 & -6.67019000 & -0.81071200 \\ \mathrm{H} & -7.60845000 & -6.36412300 & 0.91290200 \\ \mathrm{H} & -6.48083800 & -7.61223300 & 0.38332100 \\ \mathrm{H} & -7.37141900 & 6.68785300 & -0.82004000 \\ \mathrm{H} & -6.48611500 & 7.60663100 & 0.40811300 \\ \mathrm{H} & -7.62296800 & 6.34846600 & 0.89280500\end{array}$

1-H ${ }^{+}$[B3LYP/6-31G(d,p)]

$\begin{array}{rrrr}\mathrm{N} & -0.06300300 & -2.36339700 & 0.03055200 \\ \mathrm{C} & -1.18829600 & -3.12094800 & -0.18012300 \\ \mathrm{C} & 1.08436200 & -3.02691900 & -0.37571700 \\ \mathrm{~N} & -0.28185400 & 2.25778000 & -0.08611400 \\ \mathrm{C} & -1.36044000 & 3.03236700 & -0.25973100 \\ \mathrm{C} & 0.80464300 & 3.00984900 & -0.47222200 \\ \mathrm{~S} & -1.73636200 & -0.02732000 & -0.09522800 \\ \mathrm{C} & -2.96168300 & 1.20677900 & 0.17997500 \\ \mathrm{C} & -2.87944000 & -1.34380100 & 0.18395800 \\ \mathrm{C} & 2.50530800 & 1.22665200 & 0.02005200 \\ \mathrm{C} & 1.88572300 & 0.02716800 & -0.38641800 \\ \mathrm{C} & 2.13080500 & 2.58196000 & -0.34278600 \\ \mathrm{C} & 3.64147500 & 0.85466300 & 0.83731300 \\ \mathrm{C} & 4.43229400 & 1.74211700 & 1.57487900 \\ \mathrm{C} & 3.71551800 & -0.60449500 & 0.85440700 \\ \mathrm{C} & 5.50969000 & 1.48436200 & 2.42303000\end{array}$




\begin{tabular}{|c|c|c|c|}
\hline $\mathrm{H}$ & 4.17588200 & 2.79014700 & 1.47396300 \\
\hline $\mathrm{C}$ & 6.05997300 & 0.25711400 & 2.79808800 \\
\hline $\mathrm{H}$ & 5.97551200 & 2.36339000 & 2.86033400 \\
\hline $\mathrm{C}$ & 5.64282900 & -1.02316300 & 2.43715400 \\
\hline $\mathrm{H}$ & 6.90764000 & 0.30595700 & 3.47715600 \\
\hline $\mathrm{C}$ & 4.59208300 & -1.39835600 & 1.59615000 \\
\hline $\mathrm{C}$ & 2.61825400 & -1.10354700 & 0.04762300 \\
\hline $\mathrm{H}$ & 4.44248400 & -2.46837900 & 1.50742600 \\
\hline $\mathrm{H}$ & 6.19872000 & -1.84497400 & 2.88041800 \\
\hline $\mathrm{C}$ & 2.37350600 & -2.49609000 & -0.27275900 \\
\hline $\mathrm{C}$ & 3.52050000 & -3.39404700 & -0.54845300 \\
\hline $\mathrm{C}$ & 4.56891200 & -2.95508400 & -1.38122000 \\
\hline $\mathrm{C}$ & 3.59604300 & -4.69107100 & -0.00259300 \\
\hline $\mathrm{C}$ & 5.64395400 & -3.79023300 & -1.67144200 \\
\hline $\mathrm{H}$ & 4.51826900 & -1.96320300 & -1.81867100 \\
\hline $\mathrm{C}$ & 5.70417900 & -5.07468600 & -1.12468800 \\
\hline $\mathrm{H}$ & 6.43385100 & -3.44178500 & -2.32959500 \\
\hline $\mathrm{C}$ & 4.67958800 & -5.52019700 & -0.28709800 \\
\hline $\mathrm{H}$ & 6.54577900 & -5.72291900 & -1.34773600 \\
\hline $\mathrm{H}$ & 4.72747700 & -6.51182700 & 0.15213900 \\
\hline $\mathrm{H}$ & 2.81707200 & -5.03233700 & 0.67146100 \\
\hline $\mathrm{C}$ & 0.63284500 & -4.28961300 & -0.89011800 \\
\hline $\mathrm{C}$ & -0.73188600 & -4.35219100 & -0.75469400 \\
\hline $\mathrm{H}$ & 1.27124200 & -5.02858800 & -1.34951100 \\
\hline $\mathrm{H}$ & -1.38407400 & -5.15025900 & -1.07536300 \\
\hline $\mathrm{C}$ & -2.52852400 & -2.69817400 & 0.04676900 \\
\hline $\mathrm{C}$ & -3.58271600 & -3.74837200 & 0.08105600 \\
\hline $\mathrm{C}$ & -4.71520000 & -3.67219000 & -0.74826400 \\
\hline $\mathrm{C}$ & -3.46404100 & -4.85712200 & 0.93619800 \\
\hline $\mathrm{C}$ & -4.44536900 & -5.84467500 & 0.96497300 \\
\hline $\mathrm{H}$ & -2.60384000 & -4.93548400 & 1.59437300 \\
\hline $\mathrm{C}$ & -5.57887700 & -5.76704000 & 0.14437100 \\
\hline $\mathrm{H}$ & -4.33197200 & -6.68822500 & 1.64059000 \\
\hline $\mathrm{C}$ & -5.69073700 & -4.66467000 & -0.71492000 \\
\hline $\mathrm{C}$ & -6.65491800 & -6.82302200 & 0.19879500 \\
\hline $\mathrm{H}$ & -6.54951200 & -4.58859500 & -1.37652600 \\
\hline $\mathrm{H}$ & -4.81516500 & -2.84285500 & -1.44175200 \\
\hline $\mathrm{C}$ & -4.15116700 & -0.80081100 & 0.53798600 \\
\hline $\mathrm{C}$ & -4.19108400 & 0.57235000 & 0.53657000 \\
\hline $\mathrm{H}$ & -4.99436800 & -1.42821600 & 0.79588200 \\
\hline $\mathrm{H}$ & -5.07141900 & 1.14770600 & 0.79255400 \\
\hline $\mathrm{C}$ & -2.69701800 & 2.56646900 & 0.01091700 \\
\hline $\mathrm{C}$ & -3.80071500 & 3.55356400 & 0.07896800 \\
\hline $\mathrm{C}$ & -3.68345700 & 4.69889700 & 0.88812900 \\
\hline $\mathrm{C}$ & -4.98137200 & 3.39092100 & -0.66777100 \\
\hline $\mathrm{C}$ & -6.00389800 & 4.33331100 & -0.59892400 \\
\hline $\mathrm{H}$ & -5.07988600 & 2.53990300 & -1.33441000 \\
\hline $\mathrm{C}$ & -5.89540000 & 5.46597700 & 0.21919000 \\
\hline $\mathrm{H}$ & -6.89789400 & 4.19305400 & -1.20050300 \\
\hline $\mathrm{C}$ & -4.71433100 & 5.63009800 & 0.95812300 \\
\hline $\mathrm{C}$ & -7.01843600 & 6.46787900 & 0.31868100 \\
\hline $\mathrm{H}$ & -4.60271900 & 6.50006300 & 1.59971900 \\
\hline $\mathrm{H}$ & -2.78521500 & 4.84455600 & 1.47997100 \\
\hline $\mathrm{C}$ & -0.99608000 & 4.33719700 & -0.80760200 \\
\hline $\mathrm{C}$ & 0.35271000 & 4.31635100 & -0.95913100 \\
\hline $\mathrm{H}$ & -1.68122700 & 5.12812100 & -1.07647200 \\
\hline $\mathrm{H}$ & 0.97552000 & 5.08895500 & -1.38410300 \\
\hline
\end{tabular}




$\begin{array}{lrrr}\mathrm{C} & 3.22352700 & 3.55169200 & -0.61598300 \\ \mathrm{C} & 3.23210000 & 4.84136200 & -0.04867700 \\ \mathrm{C} & 4.29101700 & 3.18404300 & -1.45800100 \\ \mathrm{C} & 4.27026100 & 5.73086600 & -0.32032500 \\ \mathrm{H} & 2.43536700 & 5.12982200 & 0.62887800 \\ \mathrm{C} & 5.31283500 & 5.35629200 & -1.17048400 \\ \mathrm{H} & 4.26680900 & 6.71604600 & 0.13585500 \\ \mathrm{C} & 5.31788200 & 4.08108200 & -1.74054600 \\ \mathrm{H} & 6.11741400 & 6.05262400 & -1.38535600 \\ \mathrm{H} & 6.12163200 & 3.78673800 & -2.40838000 \\ \mathrm{H} & 4.29376200 & 2.19735600 & -1.91027700 \\ \mathrm{H} & 1.02264300 & -0.00463900 & -1.03684200 \\ \mathrm{H} & -7.13403300 & -6.95715400 & -0.77515700 \\ \mathrm{H} & -7.44063900 & -6.54457300 & 0.91176100 \\ \mathrm{H} & -6.25346400 & -7.78862400 & 0.51805100 \\ \mathrm{H} & -7.64619800 & 6.45738500 & -0.57630900 \\ \mathrm{H} & -6.63777600 & 7.48384900 & 0.45743500 \\ \mathrm{H} & -7.66603100 & 6.24470200 & 1.17550400 \\ \mathrm{H} & -0.04224900 & -1.49647900 & 0.55008900\end{array}$

1- $\mathrm{H}_{2}{ }^{2+}{ }_{\mathrm{o}}[\mathrm{B} 3 \mathrm{LYP} / 6-31 \mathrm{G}(\mathrm{d}, \mathrm{p})]$

$\begin{array}{lrrr}\mathrm{N} & 0.16432600 & 2.35282900 & -0.04206200 \\ \mathrm{C} & 1.30060000 & 3.07859300 & -0.30989700 \\ \mathrm{C} & -0.97041700 & 3.01449000 & -0.47960300 \\ \mathrm{~N} & 0.16427000 & -2.35278800 & -0.04219300 \\ \mathrm{C} & 1.30049200 & -3.07868900 & -0.30989800 \\ \mathrm{C} & -0.97051600 & -3.01443200 & -0.47960600 \\ \mathrm{~S} & 1.87443800 & -0.00003400 & -0.43973400 \\ \mathrm{C} & 2.94878500 & -1.28244500 & 0.12419400 \\ \mathrm{C} & 2.94883100 & 1.28226900 & 0.12425000 \\ \mathrm{C} & -2.56615000 & -1.16677200 & 0.05752700 \\ \mathrm{C} & -1.89592500 & 0.00003100 & -0.37764300 \\ \mathrm{C} & -2.28258600 & -2.53512600 & -0.31216000 \\ \mathrm{C} & -3.67147800 & -0.72516500 & 0.90099000 \\ \mathrm{C} & -4.48505100 & -1.57266200 & 1.66106700 \\ \mathrm{C} & -3.67140100 & 0.72537700 & 0.90099900 \\ \mathrm{C} & -5.53302000 & -1.25656400 & 2.52699400 \\ \mathrm{H} & -4.28056800 & -2.63277300 & 1.56218500 \\ \mathrm{C} & -6.01220500 & 0.00010700 & 2.90222800 \\ \mathrm{H} & -6.03427900 & -2.10735200 & 2.98017800 \\ \mathrm{C} & -5.53274900 & 1.25676300 & 2.52719800 \\ \mathrm{H} & -6.84811600 & 0.00017200 & 3.59703200 \\ \mathrm{C} & -4.48477700 & 1.57288400 & 1.66131900 \\ \mathrm{C} & -2.56609400 & 1.16689600 & 0.05753400 \\ \mathrm{H} & -4.28014500 & 2.63297700 & 1.56260700 \\ \mathrm{H} & -6.03386700 & 2.10754000 & 2.98057200 \\ \mathrm{C} & -2.28248800 & 2.53523000 & -0.31222900 \\ \mathrm{C} & -3.39815800 & 3.46800700 & -0.56726600 \\ \mathrm{C} & -4.51069200 & 3.03915300 & -1.32294100 \\ \mathrm{C} & -3.38137900 & 4.79379600 & -0.08230500 \\ \mathrm{C} & -5.55748300 & 3.91212700 & -1.59964600 \\ \mathrm{H} & -4.52641000 & 2.03045100 & -1.72289200 \\ \mathrm{C} & -5.52729100 & 5.22194400 & -1.11140600 \\ \mathrm{H} & -6.39443900 & 3.57659900 & -2.20348200 \\ \mathrm{C} & -4.44054100 & 5.65762600 & -0.34847900\end{array}$




\begin{tabular}{|c|c|c|c|}
\hline $\mathrm{H}$ & -6.34781400 & 5.89976100 & -1.32370800 \\
\hline $\mathrm{H}$ & -4.42207500 & 6.66911000 & 0.04422400 \\
\hline $\mathrm{H}$ & -2.55449400 & 5.12921300 & 0.53514000 \\
\hline $\mathrm{C}$ & -0.50178900 & 4.22243800 & -1.08909700 \\
\hline $\mathrm{C}$ & 0.86974500 & 4.26720800 & -0.97052500 \\
\hline $\mathrm{H}$ & -1.12942500 & 4.93531700 & -1.60188000 \\
\hline $\mathrm{H}$ & 1.53434700 & 5.02276400 & -1.36170000 \\
\hline $\mathrm{C}$ & 2.63540300 & 2.64703800 & -0.02446300 \\
\hline $\mathrm{C}$ & 3.69363000 & 3.66900400 & 0.07591700 \\
\hline $\mathrm{C}$ & 4.94380800 & 3.48657600 & -0.55131300 \\
\hline $\mathrm{C}$ & 3.47693400 & 4.86609200 & 0.78849000 \\
\hline $\mathrm{C}$ & 4.47439700 & 5.82946800 & 0.87766400 \\
\hline $\mathrm{H}$ & 2.53246000 & 5.02508500 & 1.29950700 \\
\hline $\mathrm{C}$ & 5.72082600 & 5.64827900 & 0.25706800 \\
\hline $\mathrm{H}$ & 4.28827400 & 6.73728800 & 1.44408100 \\
\hline $\mathrm{C}$ & 5.93010600 & 4.46065300 & -0.46335500 \\
\hline $\mathrm{C}$ & 6.80697900 & 6.68434400 & 0.37588300 \\
\hline $\mathrm{H}$ & 6.87600800 & 4.30734300 & -0.97475700 \\
\hline $\mathrm{H}$ & 5.12096400 & 2.59736200 & -1.14792400 \\
\hline $\mathrm{C}$ & 4.11646700 & 0.68974600 & 0.67787100 \\
\hline $\mathrm{C}$ & 4.11641300 & -0.68995500 & 0.67785900 \\
\hline $\mathrm{H}$ & 4.90869400 & 1.28990800 & 1.10700600 \\
\hline $\mathrm{H}$ & 4.90859500 & -1.29017200 & 1.10701600 \\
\hline $\mathrm{C}$ & 2.63533400 & -2.64718100 & -0.02452200 \\
\hline $\mathrm{C}$ & 3.69348200 & -3.66913200 & 0.07581500 \\
\hline $\mathrm{C}$ & 3.47658500 & -4.86644900 & 0.78795400 \\
\hline $\mathrm{C}$ & 4.94396300 & -3.48646800 & -0.55082300 \\
\hline $\mathrm{C}$ & 5.93019400 & -4.46054500 & -0.46285200 \\
\hline $\mathrm{H}$ & 5.12118800 & -2.59708500 & -1.14714400 \\
\hline $\mathrm{C}$ & 5.72061700 & -5.64850200 & 0.25702000 \\
\hline $\mathrm{H}$ & 6.87630300 & -4.30714400 & -0.97384000 \\
\hline $\mathrm{C}$ & 4.47397000 & -5.82986500 & 0.87715000 \\
\hline $\mathrm{C}$ & 6.80662600 & -6.68468900 & 0.37592100 \\
\hline $\mathrm{H}$ & 4.28769500 & -6.73780600 & 1.44332500 \\
\hline $\mathrm{H}$ & 2.53196200 & -5.02557800 & 1.29865700 \\
\hline $\mathrm{C}$ & 0.86956000 & -4.26726300 & -0.97054100 \\
\hline $\mathrm{C}$ & -0.50198500 & -4.22250100 & -1.08891200 \\
\hline $\mathrm{H}$ & 1.53414600 & -5.02278400 & -1.36182300 \\
\hline $\mathrm{H}$ & -1.12969400 & -4.93534400 & -1.60166000 \\
\hline $\mathrm{C}$ & -3.39827900 & -3.46787800 & -0.56720900 \\
\hline $\mathrm{C}$ & -3.38170800 & -4.79358700 & -0.08202000 \\
\hline $\mathrm{C}$ & -4.51065300 & -3.03905400 & -1.32314100 \\
\hline $\mathrm{C}$ & -4.44091200 & -5.65736400 & -0.34821200 \\
\hline $\mathrm{H}$ & -2.55496600 & -5.12897500 & 0.53562800 \\
\hline $\mathrm{C}$ & -5.52749200 & -5.22172000 & -1.11139900 \\
\hline $\mathrm{H}$ & -4.42258500 & -6.66877600 & 0.04468300 \\
\hline $\mathrm{C}$ & -5.55747100 & -3.91197900 & -1.59987600 \\
\hline $\mathrm{H}$ & -6.34804900 & -5.89948600 & -1.32373200 \\
\hline $\mathrm{H}$ & -6.39429900 & -3.57648100 & -2.20390500 \\
\hline $\mathrm{H}$ & -4.52622100 & -2.03040700 & -1.72324900 \\
\hline $\mathrm{H}$ & -1.07843100 & 0.00002600 & -1.08790000 \\
\hline $\mathrm{H}$ & 7.42415700 & 6.72317100 & -0.52562600 \\
\hline $\mathrm{H}$ & 7.47286300 & 6.44827100 & 1.21508800 \\
\hline $\mathrm{H}$ & 6.39531700 & 7.68031000 & 0.55642300 \\
\hline $\mathrm{H}$ & 7.42468600 & -6.72271100 & -0.52500500 \\
\hline $\mathrm{H}$ & 6.39468400 & -7.68082200 & 0.55515900 \\
\hline H & 7.47162200 & -6.44956300 & 1.21606900 \\
\hline
\end{tabular}




$$
\begin{array}{lrrr}
\mathrm{H} & 0.13618100 & 1.53847300 & 0.55287200 \\
\mathrm{H} & 0.13620000 & -1.53854500 & 0.55289100 \\
\text { 1-Ru(II) })_{\mathrm{o}}(\mathrm{B} 3 \mathrm{~L} \text { YP/6-31G(d,p)/LANL2DZ) }
\end{array}
$$

\begin{tabular}{|c|c|c|c|}
\hline $\mathrm{Ru}$ & -0.14212600 & 0.00000000 & -0.22102000 \\
\hline $\mathrm{Cl}$ & 0.68246100 & 0.00002000 & -2.60014700 \\
\hline $\mathrm{C}$ & 2.61961600 & 1.16158900 & 0.33231900 \\
\hline $\mathrm{C}$ & 3.90607300 & 0.73210900 & 0.84895700 \\
\hline $\mathrm{C}$ & 4.90282400 & 1.56330900 & 1.38037000 \\
\hline $\mathrm{H}$ & 4.69926600 & 2.62393900 & 1.32920700 \\
\hline $\mathrm{C}$ & 6.12033700 & 1.24754200 & 1.98653500 \\
\hline $\mathrm{H}$ & 6.70691000 & 2.10695000 & 2.30441000 \\
\hline $\mathrm{C}$ & 6.68329800 & 0.00004900 & 2.25178800 \\
\hline $\mathrm{H}$ & 7.65611100 & 0.00006100 & 2.73744300 \\
\hline $\mathrm{C}$ & 3.90609200 & -0.73206900 & 0.84895600 \\
\hline $\mathrm{C}$ & 4.90287400 & -1.56324500 & 1.38032900 \\
\hline $\mathrm{H}$ & 4.69935600 & -2.62388200 & 1.32914100 \\
\hline $\mathrm{C}$ & 6.12038900 & -1.24745200 & 1.98649000 \\
\hline $\mathrm{H}$ & 6.70699800 & -2.10685200 & 2.30432300 \\
\hline $\mathrm{C}$ & 2.61963100 & -1.16157200 & 0.33232800 \\
\hline $\mathrm{C}$ & 2.22786100 & -2.48426400 & -0.04951900 \\
\hline $\mathrm{C}$ & 3.28385400 & -3.52523700 & -0.26765000 \\
\hline $\mathrm{C}$ & 4.20437500 & -3.35736300 & -1.31481900 \\
\hline $\mathrm{H}$ & 4.13069000 & -2.47716100 & -1.94616700 \\
\hline $\mathrm{C}$ & 5.18919200 & -4.31586700 & -1.55247500 \\
\hline $\mathrm{H}$ & 5.88714500 & -4.17664500 & -2.37311900 \\
\hline $\mathrm{C}$ & 5.27683500 & -5.45132700 & -0.74358600 \\
\hline $\mathrm{H}$ & 6.04733200 & -6.19461200 & -0.92711500 \\
\hline $\mathrm{C}$ & 4.36563500 & -5.62858300 & 0.29946000 \\
\hline $\mathrm{H}$ & 4.42582900 & -6.50924200 & 0.93279000 \\
\hline $\mathrm{C}$ & 3.37169700 & -4.67595000 & 0.53235300 \\
\hline $\mathrm{H}$ & 2.66539600 & -4.81299200 & 1.34576100 \\
\hline $\mathrm{C}$ & 0.91637300 & -2.89471800 & -0.34699000 \\
\hline $\mathrm{C}$ & 0.51813800 & -4.24447600 & -0.69058600 \\
\hline $\mathrm{H}$ & 1.18672100 & -5.07301500 & -0.86473900 \\
\hline $\mathrm{C}$ & -0.84204000 & -4.25974200 & -0.74170700 \\
\hline $\mathrm{H}$ & -1.48461100 & -5.09834100 & -0.96564200 \\
\hline $\mathrm{C}$ & -1.30031500 & -2.91288800 & -0.47701100 \\
\hline $\mathrm{C}$ & -2.70459500 & -2.56909100 & -0.34595700 \\
\hline $\mathrm{C}$ & -3.65201500 & -3.66134700 & 0.00875100 \\
\hline $\mathrm{C}$ & -3.38110300 & -4.55958200 & 1.05731700 \\
\hline $\mathrm{H}$ & -2.45725200 & -4.45842000 & 1.61808200 \\
\hline $\mathrm{C}$ & -4.29057500 & -5.55696700 & 1.39735300 \\
\hline $\mathrm{H}$ & -4.06065800 & -6.22851500 & 2.22119700 \\
\hline $\mathrm{C}$ & -5.50180100 & -5.70509200 & 0.70579600 \\
\hline $\mathrm{C}$ & -5.77289500 & -4.80993300 & -0.33697400 \\
\hline $\mathrm{H}$ & -6.70221600 & -4.90322900 & -0.89375100 \\
\hline $\mathrm{C}$ & -4.86807900 & -3.80747300 & -0.68095500 \\
\hline $\mathrm{H}$ & -5.09332700 & -3.13624200 & -1.50430300 \\
\hline $\mathrm{C}$ & -3.21637900 & -1.28029400 & -0.45014900 \\
\hline $\mathrm{C}$ & -4.39503700 & -0.68952700 & 0.10845600 \\
\hline $\mathrm{H}$ & -5.15269200 & -1.28372900 & 0.60522100 \\
\hline $\mathrm{C}$ & -4.39504300 & 0.68949900 & 0.10846600 \\
\hline $\mathrm{H}$ & -5.15270400 & 1.28368700 & 0.60523700 \\
\hline $\mathrm{C}$ & -3.21639100 & 1.28028400 & -0.45013200 \\
\hline $\mathrm{C}$ & -2.70461700 & 2.56908400 & -0.34593100 \\
\hline $\mathrm{C}$ & -3.65204000 & 3.66133300 & 0.00879200 \\
\hline
\end{tabular}




$\begin{array}{llll}\mathrm{C} & -4.86811200 & 3.80746100 & -0.68089900 \\ \mathrm{H} & -5.09336800 & 3.13623400 & -1.50424900 \\ \mathrm{C} & -5.77292600 & 4.80991600 & -0.33690300 \\ \mathrm{H} & -6.70225400 & 4.90321200 & -0.89366800 \\ \mathrm{C} & -5.50182000 & 5.70507100 & 0.70587000 \\ \mathrm{C} & -4.29058800 & 5.55694300 & 1.39741300 \\ \mathrm{H} & -4.06066600 & 6.22848600 & 2.22125900 \\ \mathrm{C} & -3.38111700 & 4.55956100 & 1.05736100 \\ \mathrm{H} & -2.45725900 & 4.45839600 & 1.61811400 \\ \mathrm{C} & -1.30034000 & 2.91288900 & -0.47699100 \\ \mathrm{C} & -0.84207400 & 4.25974600 & -0.74169500 \\ \mathrm{H} & -1.48465400 & 5.09834200 & -0.96561600 \\ \mathrm{C} & 0.51810400 & 4.24448300 & -0.69060500 \\ \mathrm{H} & 1.18668100 & 5.07302100 & -0.86478100 \\ \mathrm{C} & 0.91634800 & 2.89472700 & -0.34700100 \\ \mathrm{C} & 2.22783800 & 2.48428000 & -0.04954200 \\ \mathrm{C} & 3.28383700 & 3.52523800 & -0.26770700 \\ \mathrm{C} & 3.37168000 & 4.67599100 & 0.53224100 \\ \mathrm{H} & 2.66537300 & 4.81307500 & 1.34563600 \\ \mathrm{C} & 4.36562600 & 5.62860500 & 0.29931400 \\ \mathrm{H} & 4.42582000 & 6.50929400 & 0.93260300 \\ \mathrm{C} & 5.27683900 & 5.45129100 & -0.74371300 \\ \mathrm{H} & 6.04734200 & 6.19456400 & -0.92726700 \\ \mathrm{C} & 5.18919800 & 4.31579400 & -1.55254600 \\ \mathrm{H} & 5.88715900 & 4.17652500 & -2.37317400 \\ \mathrm{C} & 4.20437200 & 3.35730700 & -1.31485400 \\ \mathrm{H} & 4.13068800 & 2.47707600 & -1.94616300 \\ \mathrm{C} & 1.79653700 & 0.00000100 & 0.19688000 \\ \mathrm{~N} & -0.21687400 & -2.10473500 & -0.28160900 \\ \mathrm{C} & -6.46764300 & -6.81096100 & 1.05801800 \\ \mathrm{H} & -6.47029000 & -7.01271700 & 2.13376000 \\ \mathrm{H} & -6.19770800 & -7.74772900 & 0.55409500 \\ \mathrm{H} & -7.48932000 & -6.56217900 & 0.75641400 \\ \mathrm{~S} & -2.30170300 & 0.00000500 & -1.24631500 \\ \mathrm{~N} & -0.21689600 & 2.10474200 & -0.28160000 \\ \mathrm{C} & -6.46766700 & 6.81093700 & 1.05809000 \\ \mathrm{H} & -6.47002300 & 7.01296700 & 2.13378000 \\ \mathrm{H} & -7.48940800 & 6.56198000 & 0.75684200 \\ \mathrm{H} & -6.19796900 & 7.74759800 & 0.55384400 \\ \mathrm{O} & -0.93832500 & -0.00004600 & 2.70525400 \\ \mathrm{C} & -0.65301200 & -0.00002200 & 1.58013200\end{array}$

1-Ru(II) ${ }_{o}{ }_{0}(\mathrm{~B} 3 \mathrm{LYP} / 6-31 \mathrm{G}(\mathrm{d}, \mathrm{p}) / \mathrm{LANL} 2 \mathrm{DZ})$

$\begin{array}{crrr}\mathrm{Ru} & 0.12438500 & -0.00053500 & -0.22174300 \\ \mathrm{C} & -2.68414900 & -1.16260700 & 0.13601400 \\ \mathrm{C} & -4.00880900 & -0.72576000 & 0.57688900 \\ \mathrm{C} & -5.02964500 & -1.55758700 & 1.05579700 \\ \mathrm{H} & -4.81816600 & -2.61701000 & 1.03010500 \\ \mathrm{C} & -6.28442400 & -1.24390300 & 1.58311400 \\ \mathrm{H} & -6.88663400 & -2.10247500 & 1.87013000 \\ \mathrm{C} & -6.86350000 & 0.00396300 & 1.80587200 \\ \mathrm{H} & -7.86549600 & 0.00451100 & 2.22709200 \\ \mathrm{C} & -4.00755200 & 0.73060400 & 0.57798800 \\ \mathrm{C} & -5.02690800 & 1.56346600 & 1.05823900 \\ \mathrm{H} & -4.81356200 & 2.62255500 & 1.03422700 \\ \mathrm{C} & -6.28223700 & 1.25115700 & 1.58507200\end{array}$




\begin{tabular}{|c|c|c|c|}
\hline $\mathrm{H}$ & -6.88294300 & 2.11033200 & 1.87343200 \\
\hline $\mathrm{C}$ & -2.68217100 & 1.16581300 & 0.13764800 \\
\hline $\mathrm{C}$ & -2.26265800 & 2.49571500 & -0.17818900 \\
\hline $\mathrm{C}$ & -3.28501400 & 3.57688300 & -0.36044100 \\
\hline $\mathrm{C}$ & -4.18121100 & 3.50952400 & -1.43966600 \\
\hline $\mathrm{H}$ & -4.12578500 & 2.67157200 & -2.12835700 \\
\hline $\mathrm{C}$ & -5.12966600 & 4.51318200 & -1.63638700 \\
\hline $\mathrm{H}$ & -5.80865400 & 4.45348800 & -2.48170100 \\
\hline $\mathrm{C}$ & -5.20425900 & 5.59245100 & -0.75279400 \\
\hline $\mathrm{H}$ & -5.94573200 & 6.37086800 & -0.90437400 \\
\hline $\mathrm{C}$ & -4.31779300 & 5.66905700 & 0.32365300 \\
\hline $\mathrm{H}$ & -4.36941000 & 6.50564500 & 1.01403500 \\
\hline $\mathrm{C}$ & -3.35872800 & 4.67267800 & 0.51536900 \\
\hline $\mathrm{H}$ & -2.67194400 & 4.73284100 & 1.35444400 \\
\hline $\mathrm{C}$ & -0.93214900 & 2.89268100 & -0.42951900 \\
\hline $\mathrm{C}$ & -0.51898700 & 4.24188200 & -0.74584800 \\
\hline $\mathrm{H}$ & -1.18133900 & 5.07574100 & -0.91752100 \\
\hline $\mathrm{C}$ & 0.84225200 & 4.25290000 & -0.78879700 \\
\hline $\mathrm{H}$ & 1.48691900 & 5.09298400 & -0.99806700 \\
\hline $\mathrm{C}$ & 1.29852200 & 2.90865300 & -0.53855100 \\
\hline $\mathrm{C}$ & 2.69791000 & 2.57916100 & -0.39695800 \\
\hline $\mathrm{C}$ & 3.64966200 & 3.67648100 & -0.07981200 \\
\hline $\mathrm{C}$ & 3.39799100 & 4.58862800 & 0.96172600 \\
\hline $\mathrm{H}$ & 2.48793800 & 4.49598100 & 1.54625200 \\
\hline $\mathrm{C}$ & 4.31625800 & 5.58788400 & 1.26889200 \\
\hline $\mathrm{H}$ & 4.10570800 & 6.27020600 & 2.08822300 \\
\hline $\mathrm{C}$ & 5.51309000 & 5.72432500 & 0.54911600 \\
\hline $\mathrm{C}$ & 5.76278100 & 4.81350900 & -0.48711300 \\
\hline $\mathrm{H}$ & 6.67987600 & 4.89863500 & -1.06395200 \\
\hline $\mathrm{C}$ & 4.85283300 & 3.80595200 & -0.79648300 \\
\hline $\mathrm{H}$ & 5.06253500 & 3.12392400 & -1.61520700 \\
\hline $\mathrm{C}$ & 3.21605900 & 1.28474700 & -0.47144800 \\
\hline $\mathrm{C}$ & 4.39862100 & 0.68859400 & 0.05758700 \\
\hline $\mathrm{H}$ & 5.17817500 & 1.28163700 & 0.51974000 \\
\hline $\mathrm{C}$ & 4.39741800 & -0.69666400 & 0.05758000 \\
\hline $\mathrm{H}$ & 5.17599500 & -1.29109200 & 0.51958700 \\
\hline $\mathrm{C}$ & 3.21387600 & -1.29078800 & -0.47150900 \\
\hline $\mathrm{C}$ & 2.69356400 & -2.58430900 & -0.39716700 \\
\hline $\mathrm{C}$ & 3.64364600 & -3.68267400 & -0.07867800 \\
\hline $\mathrm{C}$ & 4.84524200 & -3.81643400 & -0.79708100 \\
\hline $\mathrm{H}$ & 5.05374700 & -3.13841200 & -1.61943900 \\
\hline $\mathrm{C}$ & 5.75075700 & -4.82906600 & -0.49083000 \\
\hline $\mathrm{H}$ & 6.66315200 & -4.92199200 & -1.07384100 \\
\hline $\mathrm{C}$ & 5.50034100 & -5.73758000 & 0.54716600 \\
\hline $\mathrm{C}$ & 4.30202600 & -5.60077800 & 1.26450500 \\
\hline $\mathrm{H}$ & 4.08508900 & -6.28917200 & 2.07709100 \\
\hline $\mathrm{C}$ & 3.38823600 & -4.59658400 & 0.96045700 \\
\hline $\mathrm{H}$ & 2.47531200 & -4.50605200 & 1.54079400 \\
\hline $\mathrm{C}$ & 1.29373600 & -2.91144400 & -0.54003500 \\
\hline $\mathrm{C}$ & 0.83540500 & -4.25466000 & -0.79211200 \\
\hline $\mathrm{H}$ & 1.47881600 & -5.09557900 & -1.00195800 \\
\hline $\mathrm{C}$ & -0.52584900 & -4.24134400 & -0.75029100 \\
\hline $\mathrm{H}$ & -1.18948600 & -5.07386100 & -0.92350700 \\
\hline $\mathrm{C}$ & -0.93695800 & -2.89181500 & -0.43262600 \\
\hline $\mathrm{C}$ & -2.26687400 & -2.49284900 & -0.18148000 \\
\hline $\mathrm{C}$ & -3.29108900 & -3.57189900 & -0.36577900 \\
\hline $\mathrm{C}$ & -3.36701300 & -4.66901900 & 0.50818500 \\
\hline
\end{tabular}




$\begin{array}{lrrr}\mathrm{H} & -2.68061600 & -4.73178500 & 1.34738400 \\ \mathrm{C} & -4.32775400 & -5.66339900 & 0.31449700 \\ \mathrm{H} & -4.38105400 & -6.50104900 & 1.00346100 \\ \mathrm{C} & -5.21373100 & -5.58344900 & -0.76210900 \\ \mathrm{H} & -5.95650200 & -6.36032300 & -0.91524100 \\ \mathrm{C} & -5.13697500 & -4.50283200 & -1.64386600 \\ \mathrm{H} & -5.81557900 & -4.44054600 & -2.48930200 \\ \mathrm{C} & -4.18683800 & -3.50116000 & -1.44516300 \\ \mathrm{H} & -4.12971700 & -2.66216700 & -2.13244600 \\ \mathrm{C} & -1.86733300 & 0.00097700 & 0.02777900 \\ \mathrm{~N} & 0.19450600 & 2.08941400 & -0.37369600 \\ \mathrm{C} & 6.48725400 & 6.83196100 & 0.86513800 \\ \mathrm{H} & 6.48687000 & 7.07314400 & 1.93202500 \\ \mathrm{H} & 6.22386400 & 7.74961400 & 0.32468800 \\ \mathrm{H} & 7.50698000 & 6.56461800 & 0.57485400 \\ \mathrm{~S} & 2.26827300 & -0.00218900 & -1.19346900 \\ \mathrm{~N} & 0.19103500 & -2.09051900 & -0.37513000 \\ \mathrm{C} & 6.49689900 & -6.81407500 & 0.89909800 \\ \mathrm{H} & 7.16435400 & -6.48052500 & 1.70336300 \\ \mathrm{H} & 7.12387000 & -7.07581400 & 0.04246800 \\ \mathrm{H} & 5.99836200 & -7.72275900 & 1.24873400 \\ \mathrm{O} & 0.56447200 & -0.00181100 & 2.71810300 \\ \mathrm{C} & 0.40342600 & -0.00129900 & 1.57033800\end{array}$

1-Pd(II) ${ }_{\text {o }}^{+}(\mathrm{B} 3 \mathrm{LYP} / 6-31 \mathrm{G}(\mathrm{d}, \mathrm{p}) / \mathrm{LANL} 2 \mathrm{DZ})$ scrf=( solvent=chloroform $)$

$\begin{array}{lrrr}\mathrm{C} & -2.60459300 & 1.15964700 & 0.21388500 \\ \mathrm{C} & -3.88020700 & 0.72614200 & 0.78387600 \\ \mathrm{C} & -3.88015500 & -0.72631700 & 0.78394000 \\ \mathrm{C} & -2.60451800 & -1.15978100 & 0.21399300 \\ \mathrm{C} & -1.80887400 & -0.00005600 & 0.02889700 \\ \mathrm{C} & -4.84144600 & 1.56388300 & 1.36100600 \\ \mathrm{C} & -4.84133200 & -1.56404400 & 1.36121500 \\ \mathrm{C} & -6.04034800 & -1.24990400 & 2.00534400 \\ \mathrm{C} & -6.59374200 & -0.00006500 & 2.27984800 \\ \mathrm{C} & -6.04044700 & 1.24976200 & 2.00519100 \\ \mathrm{C} & -2.23312700 & 2.48561500 & -0.15919100 \\ \mathrm{C} & -2.23299500 & -2.48578200 & -0.15896000 \\ \mathrm{C} & -0.91305200 & -2.87678500 & -0.44883100 \\ \mathrm{C} & 1.31126200 & -2.89218900 & -0.51600000 \\ \mathrm{C} & 0.86116700 & -4.22725300 & -0.81145500 \\ \mathrm{C} & -0.50199100 & -4.21425900 & -0.79972500 \\ \mathrm{C} & -0.91318500 & 2.87668000 & -0.44902600 \\ \mathrm{~N} & 0.21117800 & 2.07670000 & -0.34060900 \\ \mathrm{C} & 1.31112400 & 2.89225700 & -0.51597600 \\ \mathrm{C} & 0.86096000 & 4.22726400 & -0.81155000 \\ \mathrm{C} & -0.50220400 & 4.21415900 & -0.79996100 \\ \mathrm{C} & -3.28699900 & 3.53003800 & -0.34500400 \\ \mathrm{C} & -3.31484000 & 4.69939800 & 0.43427300 \\ \mathrm{C} & -4.31166100 & 5.65686900 & 0.23879800 \\ \mathrm{C} & -5.28407600 & 5.46755600 & -0.74579600 \\ \mathrm{C} & -5.25901700 & 4.31298100 & -1.53217800 \\ \mathrm{C} & -4.27347600 & 3.34732500 & -1.32952200 \\ \mathrm{C} & -4.27339500 & -3.34769900 & -1.32905700 \\ \mathrm{C} & -5.25889900 & -4.31342100 & -1.53153900 \\ \mathrm{C} & -5.28383000 & -5.46793400 & -0.74504900\end{array}$




\begin{tabular}{|c|c|c|c|}
\hline $\mathrm{C}$ & -4.31132400 & -5.65711200 & 0.23946900 \\
\hline $\mathrm{C}$ & -3.31453100 & -4.69956400 & 0.43477600 \\
\hline $\mathrm{C}$ & 2.70601200 & -2.57186800 & -0.32459000 \\
\hline $\mathrm{C}$ & 3.21707600 & -1.28058900 & -0.38428100 \\
\hline $\mathrm{C}$ & 3.21702400 & 1.28080100 & -0.38417300 \\
\hline $\mathrm{C}$ & 4.37349600 & 0.69037800 & 0.20734700 \\
\hline $\mathrm{C}$ & 4.37352900 & -0.69017000 & 0.20728400 \\
\hline $\mathrm{C}$ & 2.70589000 & 2.57204500 & -0.32444700 \\
\hline $\mathrm{C}$ & 3.63903100 & 3.67170100 & 0.03604800 \\
\hline $\mathrm{C}$ & 4.88067100 & 3.79446100 & -0.61392800 \\
\hline $\mathrm{C}$ & 5.77370800 & 4.80344600 & -0.26245100 \\
\hline $\mathrm{C}$ & 5.46713200 & 5.72384900 & 0.75046900 \\
\hline $\mathrm{C}$ & 4.23163900 & 5.59553000 & 1.40273200 \\
\hline $\mathrm{C}$ & 3.33058900 & 4.59337100 & 1.05334100 \\
\hline $\mathrm{C}$ & 3.63928200 & -3.67146500 & 0.03577500 \\
\hline $\mathrm{C}$ & 3.33106200 & -4.59316000 & 1.05312200 \\
\hline $\mathrm{C}$ & 4.23223000 & -5.59524800 & 1.40237600 \\
\hline $\mathrm{C}$ & 5.46763800 & -5.72348500 & 0.74991300 \\
\hline $\mathrm{C}$ & 5.77399400 & -4.80305900 & -0.26304000 \\
\hline $\mathrm{C}$ & 4.88082600 & -3.79413000 & -0.61438200 \\
\hline $\mathrm{N}$ & 0.21125900 & -2.07671300 & -0.34058800 \\
\hline $\mathrm{H}$ & -4.62826800 & 2.62315000 & 1.31672800 \\
\hline $\mathrm{H}$ & -4.62807800 & -2.62330400 & 1.31710200 \\
\hline $\mathrm{H}$ & -6.61289100 & -2.10640400 & 2.35132800 \\
\hline $\mathrm{H}$ & -6.61303100 & 2.10628000 & 2.35106700 \\
\hline $\mathrm{H}$ & 1.51110100 & -5.06345000 & -1.02095200 \\
\hline $\mathrm{H}$ & -1.16387900 & -5.04215300 & -0.99994900 \\
\hline $\mathrm{H}$ & 1.51084800 & 5.06350400 & -1.02102100 \\
\hline $\mathrm{H}$ & -1.16413000 & 5.04198700 & -1.00033100 \\
\hline $\mathrm{H}$ & -4.25704000 & 2.45111100 & -1.94237200 \\
\hline $\mathrm{H}$ & -2.56554100 & -4.84570000 & 1.20689900 \\
\hline $\mathrm{H}$ & 5.12130800 & 1.28630600 & 0.71530600 \\
\hline $\mathrm{H}$ & 5.12136900 & -1.28610800 & 0.71518700 \\
\hline $\mathrm{H}$ & 2.38938700 & 4.50759100 & 1.58718400 \\
\hline $\mathrm{H}$ & 2.38993800 & -4.50743500 & 1.58711200 \\
\hline $\mathrm{H}$ & 3.97603300 & 6.28603900 & 2.20215200 \\
\hline $\mathrm{C}$ & 6.42501400 & 6.83292500 & 1.11001000 \\
\hline $\mathrm{H}$ & 6.72235600 & 4.88148500 & -0.78708400 \\
\hline $\mathrm{H}$ & 5.13565700 & 3.10344900 & -1.41178400 \\
\hline $\mathrm{H}$ & -2.56591600 & 4.84563000 & 1.20644400 \\
\hline $\mathrm{H}$ & -4.32701300 & 6.54982400 & 0.85639100 \\
\hline $\mathrm{H}$ & -6.05593300 & 6.21541800 & -0.90008400 \\
\hline $\mathrm{H}$ & -6.00711700 & 4.16339000 & -2.30488700 \\
\hline $\mathrm{H}$ & -4.32656500 & -6.55000600 & 0.85715300 \\
\hline $\mathrm{H}$ & -6.05566700 & -6.21584100 & -0.89921900 \\
\hline $\mathrm{H}$ & -6.00707800 & -4.16395100 & -2.30419500 \\
\hline $\mathrm{H}$ & -4.25706100 & -2.45153100 & -1.94197700 \\
\hline $\mathrm{H}$ & 3.97680100 & -6.28576300 & 2.20184800 \\
\hline $\mathrm{C}$ & 6.42560100 & -6.83253300 & 1.10932500 \\
\hline $\mathrm{H}$ & 6.72256700 & -4.88102400 & -0.78781500 \\
\hline $\mathrm{H}$ & 5.13565200 & -3.10308700 & -1.41226300 \\
\hline $\mathrm{Pd}$ & 0.12232800 & -0.00004100 & -0.38177500 \\
\hline $\mathrm{H}$ & 6.25617400 & 7.71477100 & 0.47946600 \\
\hline $\mathrm{H}$ & 6.29940600 & 7.14768000 & 2.14975400 \\
\hline $\mathrm{H}$ & 7.46500400 & 6.52611200 & 0.96611900 \\
\hline $\mathrm{H}$ & 6.25526200 & -7.71515200 & 0.48026700 \\
\hline $\mathrm{H}$ & 7.46549200 & -6.52641300 & 0.96330600 \\
\hline
\end{tabular}




$\begin{array}{lccc}\mathrm{H} & 6.30156300 & -7.14585700 & 2.14969600 \\ \mathrm{~S} & 2.32351500 & 0.00011900 & -1.19606300 \\ \mathrm{C} & -3.28682400 & -3.53027500 & -0.34460000 \\ \mathrm{H} & -7.55035700 & -0.00009100 & 2.79522300\end{array}$

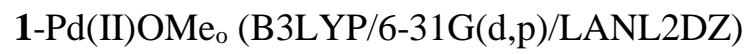

\begin{tabular}{|c|c|c|c|}
\hline $\mathrm{C}$ & -2.33251200 & 1.05800900 & 0.23151000 \\
\hline $\mathrm{C}$ & -3.68368300 & 0.55881600 & 0.62787400 \\
\hline $\mathrm{C}$ & -3.63843800 & -0.83417600 & 0.62920600 \\
\hline $\mathrm{C}$ & -2.25756500 & -1.24436100 & 0.23046800 \\
\hline $\mathrm{C}$ & -1.48286800 & -0.06669100 & 0.07873200 \\
\hline $\mathrm{C}$ & -4.74627800 & 1.34579400 & 1.20695500 \\
\hline $\mathrm{C}$ & -4.64920700 & -1.69178400 & 1.20121700 \\
\hline $\mathrm{C}$ & -5.97193100 & -1.41267600 & 1.23092600 \\
\hline $\mathrm{C}$ & -6.54001000 & -0.23825600 & 0.49280100 \\
\hline $\mathrm{C}$ & -6.04619100 & 0.97433200 & 1.23959500 \\
\hline $\mathrm{C}$ & -1.98713000 & 2.39252800 & -0.04898100 \\
\hline $\mathrm{C}$ & -1.82596300 & -2.55297100 & -0.05490500 \\
\hline $\mathrm{C}$ & -0.47979500 & -2.91932700 & -0.32698700 \\
\hline $\mathrm{C}$ & 1.74191300 & -2.86450300 & -0.46157500 \\
\hline $\mathrm{C}$ & 1.33186800 & -4.22683600 & -0.65357400 \\
\hline $\mathrm{C}$ & -0.03309900 & -4.25990800 & -0.58875200 \\
\hline $\mathrm{C}$ & -0.66744900 & 2.84824100 & -0.31597500 \\
\hline $\mathrm{N}$ & 0.47213700 & 2.08023000 & -0.29638200 \\
\hline $\mathrm{C}$ & 1.55302800 & 2.93911700 & -0.44963900 \\
\hline $\mathrm{C}$ & 1.05503400 & 4.27272100 & -0.63539900 \\
\hline $\mathrm{C}$ & -0.30923500 & 4.21629200 & -0.57121800 \\
\hline $\mathrm{C}$ & -3.05475300 & 3.44158400 & -0.12687100 \\
\hline $\mathrm{C}$ & -3.12345500 & 4.49047400 & 0.80388900 \\
\hline $\mathrm{C}$ & -4.12113100 & 5.46197500 & 0.70908400 \\
\hline $\mathrm{C}$ & -5.05653000 & 5.40817900 & -0.32605600 \\
\hline $\mathrm{C}$ & -4.99047700 & 4.37495300 & -1.26301400 \\
\hline $\mathrm{C}$ & -4.00175900 & 3.39704500 & -1.16104400 \\
\hline $\mathrm{C}$ & -3.77896500 & -3.68357800 & -1.15693000 \\
\hline $\mathrm{C}$ & -4.70219100 & -4.72376100 & -1.25484500 \\
\hline $\mathrm{C}$ & -4.69058000 & -5.76392600 & -0.32336000 \\
\hline $\mathrm{C}$ & -3.74341900 & -5.76151100 & 0.70241600 \\
\hline $\mathrm{C}$ & -2.81168900 & -4.72629800 & 0.79389100 \\
\hline $\mathrm{C}$ & 3.12609400 & -2.48805000 & -0.34066400 \\
\hline $\mathrm{C}$ & 3.60172200 & -1.18325400 & -0.43384600 \\
\hline $\mathrm{C}$ & 3.51829600 & 1.38226400 & -0.43040600 \\
\hline $\mathrm{C}$ & 4.72263100 & 0.82844900 & 0.09419800 \\
\hline $\mathrm{C}$ & 4.76750700 & -0.55368800 & 0.09252900 \\
\hline $\mathrm{C}$ & 2.95865500 & 2.65306800 & -0.33238400 \\
\hline $\mathrm{C}$ & 3.88250500 & 3.77486500 & -0.00004300 \\
\hline $\mathrm{C}$ & 5.06167200 & 3.97196100 & -0.73841400 \\
\hline $\mathrm{C}$ & 5.94805100 & 4.99765700 & -0.41530800 \\
\hline $\mathrm{C}$ & 5.69520100 & 5.86336500 & 0.65665400 \\
\hline $\mathrm{C}$ & 4.52234600 & 5.66170600 & 1.39852300 \\
\hline $\mathrm{C}$ & 3.63065000 & 4.64139900 & 1.07854100 \\
\hline $\mathrm{C}$ & 4.11951900 & -3.54871700 & -0.00862000 \\
\hline $\mathrm{C}$ & 3.91859600 & -4.43585500 & 1.06393500 \\
\hline $\mathrm{C}$ & 4.87261400 & -5.39817100 & 1.38363700 \\
\hline $\mathrm{C}$ & 6.06013100 & -5.51919900 & 0.64754200 \\
\hline $\mathrm{C}$ & 6.26230800 & -4.63259200 & -0.41808500 \\
\hline $\mathrm{C}$ & 5.31318200 & -3.66460400 & -0.74100900 \\
\hline $\mathrm{N}$ & 0.60725000 & -2.07860100 & -0.30533300 \\
\hline
\end{tabular}




$\begin{array}{lrrr}\mathrm{H} & -4.46647800 & 2.27173700 & 1.69938400 \\ \mathrm{H} & -4.31254000 & -2.60577000 & 1.67925500 \\ \mathrm{H} & -6.66888000 & -2.02419700 & 1.79763800 \\ \mathrm{H} & -6.76905800 & 1.51988400 & 1.84198500 \\ \mathrm{H} & 2.00264600 & -5.05372600 & -0.83235400 \\ \mathrm{H} & -0.67212700 & -5.12160000 & -0.70330000 \\ \mathrm{H} & 1.67063100 & 5.14242000 & -0.80969000 \\ \mathrm{H} & -1.00309900 & 5.03489800 & -0.68263000 \\ \mathrm{H} & -3.95622500 & 2.58984100 & -1.88569700 \\ \mathrm{H} & -2.07619700 & -4.72486000 & 1.59295800 \\ \mathrm{H} & 5.48010100 & 1.44894300 & 0.55739000 \\ \mathrm{H} & 5.56372000 & -1.12478100 & 0.55426500 \\ \mathrm{H} & 2.73613100 & 4.49860500 & 1.67640100 \\ \mathrm{H} & 3.01342600 & -4.35440500 & 1.65719100 \\ \mathrm{H} & 4.30896500 & 6.30922100 & 2.24564200 \\ \mathrm{C} & 6.64029900 & 6.99340300 & 0.98809000 \\ \mathrm{H} & 6.84860200 & 5.13162700 & -1.00974900 \\ \mathrm{H} & 5.27388700 & 3.32018700 & -1.58074000 \\ \mathrm{H} & -2.39652300 & 4.53353000 & 1.60962200 \\ \mathrm{H} & -4.16572600 & 6.26121900 & 1.44364600 \\ \mathrm{H} & -5.83029000 & 6.16675600 & -0.40242600 \\ \mathrm{H} & -5.71102200 & 4.32901400 & -2.07475500 \\ \mathrm{H} & -3.72842800 & -6.56542200 & 1.43307000 \\ \mathrm{H} & -5.41446500 & -6.57050300 & -0.39607200 \\ \mathrm{H} & -5.43392200 & -4.71983000 & -2.05765700 \\ \mathrm{H} & -3.79447800 & -2.87101800 & -1.87678100 \\ \mathrm{H} & 4.69664800 & -6.06284300 & 2.22605200 \\ \mathrm{C} & 7.07409300 & -6.58805400 & 0.97824600 \\ \mathrm{H} & 7.17289700 & -4.70468700 & -1.00796400 \\ \mathrm{H} & 5.48750700 & -2.99628500 & -1.57907600 \\ \mathrm{Pd} & 0.44178700 & -0.00242200 & -0.35134300 \\ \mathrm{H} & 6.33224900 & 7.92414200 & 0.49500100 \\ \mathrm{H} & 6.66571300 & 7.19141400 & 2.06411900 \\ \mathrm{H} & 7.66002600 & 6.77328600 & 0.65865500 \\ \mathrm{H} & 6.82788100 & -7.53432200 & 0.47998300 \\ \mathrm{H} & 8.07899600 & -6.30193600 & 0.65406000 \\ \mathrm{H} & 7.10783700 & -6.78891300 & 2.05351700 \\ \mathrm{~S} & 2.62840100 & 0.07027100 & -1.19021400 \\ \mathrm{C} & -2.82152400 & -3.67051000 & -0.13159600 \\ \mathrm{H} & -6.13795800 & -0.22142600 & -0.53795800 \\ \mathrm{O} & -7.95583100 & -0.34387500 & 0.47092700 \\ \mathrm{C} & -8.56853000 & 0.57086400 & -0.41394100 \\ \mathrm{H} & -9.64749300 & 0.40869000 & -0.34871200 \\ \mathrm{H} & -8.35154500 & 1.61702000 & -0.15191500 \\ \mathrm{H} & -8.24646000 & 0.40594900 & -1.45469500\end{array}$

1-Ru(II)- $1_{o}(\mathrm{BP} 86 / 6-31 \mathrm{G}(\mathrm{d}, \mathrm{p}) / \mathrm{LANL} 2 \mathrm{DZ})$

$\begin{array}{crrr}\mathrm{Ru} & 0.14369000 & 0.00000000 & -0.20124200 \\ \mathrm{Cl} & -0.63155100 & -0.00000300 & -2.57558000 \\ \mathrm{C} & -2.63256600 & -1.16537200 & 0.31195100 \\ \mathrm{C} & -3.91805600 & -0.73339400 & 0.84669500 \\ \mathrm{C} & -4.90732000 & -1.57544300 & 1.39874300 \\ \mathrm{H} & -4.69848400 & -2.64546200 & 1.33597200 \\ \mathrm{C} & -6.11639800 & -1.25660200 & 2.03676600 \\ \mathrm{H} & -6.70184400 & -2.12218800 & 2.37100900\end{array}$




\begin{tabular}{|c|c|c|c|}
\hline $\mathrm{C}$ & -6.67566900 & -0.00000300 & 2.31584500 \\
\hline $\mathrm{H}$ & -7.64531800 & -0.00000300 & 2.82750600 \\
\hline $\mathrm{C}$ & -3.91805600 & 0.73339000 & 0.84669500 \\
\hline $\mathrm{C}$ & -4.90732100 & 1.57543900 & 1.39874200 \\
\hline $\mathrm{H}$ & -4.69848600 & 2.64545800 & 1.33597000 \\
\hline $\mathrm{C}$ & -6.11639900 & 1.25659700 & 2.03676500 \\
\hline $\mathrm{H}$ & -6.70184600 & 2.12218300 & 2.37100800 \\
\hline $\mathrm{C}$ & -2.63256800 & 1.16536800 & 0.31195000 \\
\hline $\mathrm{C}$ & -2.24751400 & 2.49218200 & -0.06964700 \\
\hline $\mathrm{C}$ & -3.30760600 & 3.52802000 & -0.29551000 \\
\hline $\mathrm{C}$ & -4.26609000 & 3.32403900 & -1.31433200 \\
\hline $\mathrm{H}$ & -4.20968300 & 2.41425400 & -1.92024000 \\
\hline $\mathrm{C}$ & -5.26077300 & 4.28187500 & -1.55879200 \\
\hline $\mathrm{H}$ & -5.98844200 & 4.11415100 & -2.35980800 \\
\hline $\mathrm{C}$ & -5.32252900 & 5.45293800 & -0.78486800 \\
\hline $\mathrm{H}$ & -6.10294200 & 6.19740300 & -0.97395800 \\
\hline $\mathrm{C}$ & -4.37608500 & 5.66571500 & 0.23094600 \\
\hline $\mathrm{H}$ & -4.41833300 & 6.57480800 & 0.84033300 \\
\hline $\mathrm{C}$ & -3.37193900 & 4.71420200 & 0.47091100 \\
\hline $\mathrm{H}$ & -2.63879200 & 4.87507300 & 1.26805200 \\
\hline $\mathrm{C}$ & -0.92224900 & 2.89972900 & -0.35665700 \\
\hline $\mathrm{C}$ & -0.52243900 & 4.25174200 & -0.71164600 \\
\hline $\mathrm{H}$ & -1.20043300 & 5.07917700 & -0.90965600 \\
\hline $\mathrm{C}$ & 0.84710100 & 4.27035900 & -0.74614900 \\
\hline $\mathrm{H}$ & 1.49705500 & 5.11206200 & -0.97973800 \\
\hline $\mathrm{C}$ & 1.30991300 & 2.92298500 & -0.45917200 \\
\hline $\mathrm{C}$ & 2.71056200 & 2.59080800 & -0.31027800 \\
\hline $\mathrm{C}$ & 3.66424600 & 3.68837500 & 0.00960700 \\
\hline $\mathrm{C}$ & 3.37380300 & 4.66008700 & 0.99822500 \\
\hline $\mathrm{H}$ & 2.42414500 & 4.60292400 & 1.53929500 \\
\hline $\mathrm{C}$ & 4.29497100 & 5.66709100 & 1.30829700 \\
\hline $\mathrm{H}$ & 4.04983200 & 6.39752100 & 2.08839400 \\
\hline $\mathrm{C}$ & 5.53927800 & 5.75212200 & 0.64796800 \\
\hline $\mathrm{C}$ & 5.83090800 & 4.78160400 & -0.33222600 \\
\hline $\mathrm{H}$ & 6.78852500 & 4.82567600 & -0.86427400 \\
\hline $\mathrm{C}$ & 4.91533100 & 3.76956500 & -0.64707400 \\
\hline $\mathrm{H}$ & 5.15531100 & 3.03796400 & -1.42559900 \\
\hline $\mathrm{C}$ & 3.22611100 & 1.28177000 & -0.36956100 \\
\hline $\mathrm{C}$ & 4.38106900 & 0.69764200 & 0.23367100 \\
\hline $\mathrm{H}$ & 5.12295300 & 1.29976900 & 0.76367200 \\
\hline $\mathrm{C}$ & 4.38107000 & -0.69763700 & 0.23367100 \\
\hline $\mathrm{H}$ & 5.12295500 & -1.29976300 & 0.76367300 \\
\hline $\mathrm{C}$ & 3.22611200 & -1.28176600 & -0.36956100 \\
\hline $\mathrm{C}$ & 2.71056500 & -2.59080500 & -0.31027600 \\
\hline $\mathrm{C}$ & 3.66425000 & -3.68837100 & 0.00960900 \\
\hline $\mathrm{C}$ & 4.91533600 & -3.76956000 & -0.64707300 \\
\hline $\mathrm{H}$ & 5.15531400 & -3.03795800 & -1.42559900 \\
\hline $\mathrm{C}$ & 5.83091500 & -4.78159600 & -0.33222600 \\
\hline $\mathrm{H}$ & 6.78853100 & -4.82566500 & -0.86427500 \\
\hline $\mathrm{C}$ & 5.53928600 & -5.75211500 & 0.64797000 \\
\hline $\mathrm{C}$ & 4.29498100 & -5.66708500 & 1.30830000 \\
\hline $\mathrm{H}$ & 4.04984600 & -6.39751400 & 2.08839800 \\
\hline $\mathrm{C}$ & 3.37381000 & -4.66008100 & 0.99822800 \\
\hline $\mathrm{H}$ & 2.42415400 & -4.60291900 & 1.53929900 \\
\hline $\mathrm{C}$ & 1.30991600 & -2.92298400 & -0.45917000 \\
\hline $\mathrm{C}$ & 0.84710600 & -4.27035800 & -0.74614700 \\
\hline $\mathrm{H}$ & 1.49706100 & -5.11206000 & -0.97973600 \\
\hline
\end{tabular}




$\begin{array}{lrrr}\mathrm{C} & -0.52243400 & -4.25174300 & -0.71164300 \\ \mathrm{H} & -1.20042700 & -5.07917900 & -0.90965300 \\ \mathrm{C} & -0.92224600 & -2.89973000 & -0.35665500 \\ \mathrm{C} & -2.24751100 & -2.49218500 & -0.06964500 \\ \mathrm{C} & -3.30760100 & -3.52802500 & -0.29550700 \\ \mathrm{C} & -3.37193100 & -4.71420700 & 0.47091300 \\ \mathrm{H} & -2.63878300 & -4.87507700 & 1.26805400 \\ \mathrm{C} & -4.37607600 & -5.66572100 & 0.23095000 \\ \mathrm{H} & -4.41832100 & -6.57481500 & 0.84033700 \\ \mathrm{C} & -5.32252200 & -5.45294600 & -0.78486300 \\ \mathrm{H} & -6.10293300 & -6.19741300 & -0.97395200 \\ \mathrm{C} & -5.26076800 & -4.28188300 & -1.55878700 \\ \mathrm{H} & -5.98843900 & -4.11416000 & -2.35980200 \\ \mathrm{C} & -4.26608700 & -3.32404500 & -1.31432800 \\ \mathrm{H} & -4.20968200 & -2.41426000 & -1.92023600 \\ \mathrm{C} & -1.79988500 & -0.00000100 & 0.17676900 \\ \mathrm{~N} & 0.21151900 & 2.10127600 & -0.26996000 \\ \mathrm{C} & 6.51532500 & 6.86416200 & 0.96439500 \\ \mathrm{H} & 6.48191400 & 7.14051700 & 2.03165000 \\ \mathrm{H} & 6.28092000 & 7.77723600 & 0.38593100 \\ \mathrm{H} & 7.55057700 & 6.57774400 & 0.71633500 \\ \mathrm{~S} & 2.31143900 & 0.00000100 & -1.17554000 \\ \mathrm{~N} & 0.21152200 & -2.10127600 & -0.26995900 \\ \mathrm{C} & 6.51533600 & -6.86415500 & 0.96438400 \\ \mathrm{H} & 6.48168400 & -7.14075500 & 2.03156800 \\ \mathrm{H} & 7.55062900 & -6.57760700 & 0.71664100 \\ \mathrm{H} & 6.28114300 & -7.77711300 & 0.38565000 \\ \mathrm{O} & 0.89752600 & 0.00001400 & 2.74767300 \\ \mathrm{C} & 0.62635900 & -0.00000200 & 1.60349100\end{array}$

1-Ru(II)- $t_{o}(\mathrm{BP} 86 / 6-31 \mathrm{G}(\mathrm{d}, \mathrm{p}) / \mathrm{LANL} 2 \mathrm{DZ})$

$\begin{array}{crrr}\mathrm{Ru} & 0.02476900 & 0.00000000 & 0.31373500 \\ \mathrm{C} & -2.75953200 & -1.17491200 & 0.18681400 \\ \mathrm{C} & -4.13271100 & -0.73576900 & 0.42603400 \\ \mathrm{C} & -5.22315500 & -1.57098900 & 0.74610700 \\ \mathrm{H} & -5.00391600 & -2.64013300 & 0.75120800 \\ \mathrm{C} & -6.55171900 & -1.25492800 & 1.07349400 \\ \mathrm{H} & -7.19640000 & -2.12178900 & 1.26412700 \\ \mathrm{C} & -7.16398700 & -0.00000400 & 1.20443400 \\ \mathrm{H} & -8.22845500 & -0.00000500 & 1.46725500 \\ \mathrm{C} & -4.13271200 & 0.73576500 & 0.42603300 \\ \mathrm{C} & -5.22315800 & 1.57098300 & 0.74610200 \\ \mathrm{H} & -5.00392200 & 2.64012700 & 0.75120000 \\ \mathrm{C} & -6.55172200 & 1.25492100 & 1.07348900 \\ \mathrm{H} & -7.19640500 & 2.12178000 & 1.26411800 \\ \mathrm{C} & -2.75953400 & 1.17490900 & 0.18681500 \\ \mathrm{C} & -2.27923800 & 2.51306000 & -0.00671200 \\ \mathrm{C} & -3.25643900 & 3.60033300 & -0.34859400 \\ \mathrm{C} & -3.97517600 & 3.53340600 & -1.56389100 \\ \mathrm{H} & -3.80278100 & 2.68651800 & -2.23570000 \\ \mathrm{C} & -4.88487200 & 4.54303800 & -1.91107800 \\ \mathrm{H} & -5.42675100 & 4.48066000 & -2.86074000 \\ \mathrm{C} & -5.09692100 & 5.63241700 & -1.04935900 \\ \mathrm{H} & -5.81011700 & 6.41800300 & -1.32003600 \\ \mathrm{C} & -4.38518000 & 5.71167900 & 0.15921600\end{array}$




\begin{tabular}{|c|c|c|c|}
\hline $\mathrm{H}$ & -4.54377600 & 6.55792800 & 0.83622700 \\
\hline $\mathrm{C}$ & -3.46709300 & 4.70713400 & 0.50508900 \\
\hline $\mathrm{H}$ & -2.91493000 & 4.76497800 & 1.44889600 \\
\hline $\mathrm{C}$ & -0.91443700 & 2.91651300 & -0.02791700 \\
\hline $\mathrm{C}$ & -0.46594400 & 4.27002700 & -0.30718900 \\
\hline $\mathrm{H}$ & -1.11068800 & 5.11226800 & -0.54815400 \\
\hline $\mathrm{C}$ & 0.90027900 & 4.26787200 & -0.24365000 \\
\hline $\mathrm{H}$ & 1.56877100 & 5.10657000 & -0.42606400 \\
\hline $\mathrm{C}$ & 1.33910900 & 2.91013400 & 0.04713400 \\
\hline $\mathrm{C}$ & 2.75882500 & 2.59499500 & 0.07030200 \\
\hline $\mathrm{C}$ & 3.73265000 & 3.73032600 & 0.02415500 \\
\hline $\mathrm{C}$ & 3.76128700 & 4.71044000 & 1.04249000 \\
\hline $\mathrm{H}$ & 3.04093500 & 4.64687900 & 1.86464100 \\
\hline $\mathrm{C}$ & 4.70805400 & 5.74242000 & 1.01807400 \\
\hline $\mathrm{H}$ & 4.71532400 & 6.48420500 & 1.82550000 \\
\hline $\mathrm{C}$ & 5.65908900 & 5.83936800 & -0.01932600 \\
\hline $\mathrm{C}$ & 5.63027000 & 4.85918200 & -1.03145200 \\
\hline $\mathrm{H}$ & 6.35456100 & 4.91280300 & -1.85281300 \\
\hline $\mathrm{C}$ & 4.68666000 & 3.82217100 & -1.01370600 \\
\hline $\mathrm{H}$ & 4.67018600 & 3.08329400 & -1.82165200 \\
\hline $\mathrm{C}$ & 3.32607000 & 1.29617500 & 0.09440700 \\
\hline $\mathrm{C}$ & 4.64278400 & 0.69911000 & 0.03390300 \\
\hline $\mathrm{H}$ & 5.55399300 & 1.29799700 & -0.02260500 \\
\hline $\mathrm{C}$ & 4.64278500 & -0.69910600 & 0.03390300 \\
\hline $\mathrm{H}$ & 5.55399400 & -1.29799300 & -0.02260500 \\
\hline $\mathrm{C}$ & 3.32607100 & -1.29617300 & 0.09440700 \\
\hline $\mathrm{C}$ & 2.75882700 & -2.59499300 & 0.07030200 \\
\hline $\mathrm{C}$ & 3.73265200 & -3.73032300 & 0.02415500 \\
\hline $\mathrm{C}$ & 4.68666400 & -3.82216900 & -1.01370300 \\
\hline $\mathrm{H}$ & 4.67019100 & -3.08329100 & -1.82164900 \\
\hline $\mathrm{C}$ & 5.63027500 & -4.85918000 & -1.03144700 \\
\hline $\mathrm{H}$ & 6.35456800 & -4.91280000 & -1.85280500 \\
\hline $\mathrm{C}$ & 5.65909300 & -5.83936400 & -0.01932100 \\
\hline $\mathrm{C}$ & 4.70805600 & -5.74241600 & 1.01807800 \\
\hline $\mathrm{H}$ & 4.71532500 & -6.48420000 & 1.82550600 \\
\hline $\mathrm{C}$ & 3.76128900 & -4.71043800 & 1.04249200 \\
\hline $\mathrm{H}$ & 3.04093500 & -4.64687700 & 1.86464100 \\
\hline $\mathrm{C}$ & 1.33911100 & -2.91013300 & 0.04713300 \\
\hline $\mathrm{C}$ & 0.90028300 & -4.26787100 & -0.24365400 \\
\hline $\mathrm{H}$ & 1.56877600 & -5.10656800 & -0.42606800 \\
\hline $\mathrm{C}$ & -0.46594000 & -4.27002700 & -0.30719300 \\
\hline $\mathrm{H}$ & -1.11068300 & -5.11226800 & -0.54816000 \\
\hline $\mathrm{C}$ & -0.91443400 & -2.91651400 & -0.02791900 \\
\hline $\mathrm{C}$ & -2.27923600 & -2.51306200 & -0.00671500 \\
\hline $\mathrm{C}$ & -3.25643700 & -3.60033400 & -0.34859900 \\
\hline $\mathrm{C}$ & -3.46708700 & -4.70714100 & 0.50507800 \\
\hline $\mathrm{H}$ & -2.91492100 & -4.76498900 & 1.44888300 \\
\hline $\mathrm{C}$ & -4.38517400 & -5.71168500 & 0.15920200 \\
\hline $\mathrm{H}$ & -4.54376700 & -6.55793700 & 0.83620800 \\
\hline $\mathrm{C}$ & -5.09691900 & -5.63241500 & -1.04937000 \\
\hline $\mathrm{H}$ & -5.81011600 & -6.41800100 & -1.32005000 \\
\hline $\mathrm{C}$ & -4.88487400 & -4.54303100 & -1.91108400 \\
\hline $\mathrm{H}$ & -5.42675600 & -4.48064800 & -2.86074400 \\
\hline $\mathrm{C}$ & -3.97517700 & -3.53340100 & -1.56389400 \\
\hline $\mathrm{H}$ & -3.80278600 & -2.68650900 & -2.23569800 \\
\hline $\mathrm{C}$ & -1.93564800 & -0.00000100 & 0.19198100 \\
\hline $\mathrm{N}$ & 0.19642700 & 2.11024100 & 0.17884600 \\
\hline
\end{tabular}




$\begin{array}{lrcc}\mathrm{C} & 6.65844100 & 6.97555200 & -0.05731700 \\ \mathrm{H} & 7.56343100 & 6.70026600 & -0.62355600 \\ \mathrm{H} & 6.96771900 & 7.27683500 & 0.95758200 \\ \mathrm{H} & 6.22778000 & 7.87029400 & -0.54422800 \\ \mathrm{~S} & 2.23431100 & 0.00000100 & 0.22045000 \\ \mathrm{~N} & 0.19642800 & -2.11024200 & 0.17884500 \\ \mathrm{C} & 6.65844300 & -6.97555000 & -0.05730500 \\ \mathrm{H} & 6.22766300 & -7.87040900 & -0.54389300 \\ \mathrm{H} & 6.96798800 & -7.27659900 & 0.95758400 \\ \mathrm{H} & 7.56328300 & -6.70038400 & -0.62383900 \\ \mathrm{Cl} & -0.03854300 & 0.00000000 & -2.18759000 \\ \mathrm{O} & -0.25673400 & -0.00000100 & 3.35344500 \\ \mathrm{C} & -0.11560700 & -0.00000100 & 2.18850100\end{array}$

1-Ru(II)-2 ${ }_{o}(\mathrm{BP} 86 / 6-31 \mathrm{G}(\mathrm{d}, \mathrm{p}) / \mathrm{LANL} 2 \mathrm{DZ})$

$\begin{array}{lrrr}\mathrm{Ru} & -0.02938600 & -0.00003800 & 0.63815900 \\ \mathrm{Cl} & -0.77423800 & -0.00013800 & -1.72324400 \\ \mathrm{C} & 2.74944500 & 1.16832400 & 0.08782500 \\ \mathrm{C} & 4.14283200 & 0.73367300 & 0.12117000 \\ \mathrm{C} & 5.27450900 & 1.56562400 & 0.26296500 \\ \mathrm{H} & 5.06374400 & 2.63320600 & 0.32452200 \\ \mathrm{C} & 6.64109200 & 1.25349900 & 0.34098500 \\ \mathrm{H} & 7.30580400 & 2.12287000 & 0.41775800 \\ \mathrm{C} & 7.27095800 & 0.00108600 & 0.35425700 \\ \mathrm{H} & 8.36544400 & 0.00124300 & 0.41909000 \\ \mathrm{C} & 4.14304500 & -0.73233800 & 0.12180900 \\ \mathrm{C} & 5.27480500 & -1.56390800 & 0.26525200 \\ \mathrm{H} & 5.06413900 & -2.63142300 & 0.32846800 \\ \mathrm{C} & 6.64131000 & -1.25146200 & 0.34317900 \\ \mathrm{H} & 7.30613900 & -2.12059100 & 0.42165000 \\ \mathrm{C} & 2.74984000 & -1.16746800 & 0.08832500 \\ \mathrm{C} & 2.27617400 & -2.52135600 & 0.05411800 \\ \mathrm{C} & 3.20156100 & -3.63115400 & -0.35923800 \\ \mathrm{C} & 3.68624400 & -3.67330100 & -1.68572100 \\ \mathrm{H} & 3.38347500 & -2.88904600 & -2.38692700 \\ \mathrm{C} & 4.53753900 & -4.70797100 & -2.10132500 \\ \mathrm{H} & 4.89884900 & -4.72969900 & -3.13493700 \\ \mathrm{C} & 4.92235200 & -5.71407500 & -1.19934000 \\ \mathrm{H} & 5.58996700 & -6.51894800 & -1.52404900 \\ \mathrm{C} & 4.44261900 & -5.68464600 & 0.12078100 \\ \mathrm{H} & 4.73700600 & -6.46529200 & 0.83035400 \\ \mathrm{C} & 3.58384900 & -4.65489500 & 0.53731300 \\ \mathrm{H} & 3.21455500 & -4.62674300 & 1.56777500 \\ \mathrm{C} & 0.93786500 & -2.92940700 & 0.26509600 \\ \mathrm{C} & 0.48989600 & -4.31010600 & 0.18011000 \\ \mathrm{H} & 1.12875600 & -5.17291700 & 0.00791500 \\ \mathrm{C} & -0.86611000 & -4.30270400 & 0.34622500 \\ \mathrm{H} & -1.53935600 & -5.15739500 & 0.35351200 \\ \mathrm{C} & -1.28388900 & -2.91744100 & 0.49366200 \\ \mathrm{C} & -2.68360500 & -2.56272200 & 0.45599700 \\ \mathrm{C} & -3.66610100 & -3.61398100 & 0.04326000 \\ \mathrm{C} & -4.78797100 & -3.89954700 & 0.85257800 \\ \mathrm{H} & -4.90538000 & -3.37300600 & 1.80575200 \\ \mathrm{C} & -5.73665300 & -4.85280100 & 0.45616400 \\ \mathrm{H} & -6.59369000 & -5.06326000 & 1.10678800 \\ & & & \\ & & & \end{array}$




\begin{tabular}{|c|c|c|c|}
\hline $\mathrm{C}$ & -5.60668700 & -5.54773900 & -0.76288200 \\
\hline $\mathrm{C}$ & -4.49389600 & -5.24809100 & -1.57767300 \\
\hline $\mathrm{H}$ & -4.38087500 & -5.75747100 & -2.54200100 \\
\hline $\mathrm{C}$ & -3.53889400 & -4.30184000 & -1.18616100 \\
\hline $\mathrm{H}$ & -2.69474900 & -4.06758300 & -1.84230200 \\
\hline $\mathrm{C}$ & -3.21228300 & -1.27738500 & 0.63540100 \\
\hline $\mathrm{C}$ & -4.41723000 & -0.69659600 & 0.12900100 \\
\hline $\mathrm{H}$ & -5.19417200 & -1.30171300 & -0.34292900 \\
\hline $\mathrm{C}$ & -4.41746200 & 0.69511200 & 0.12914800 \\
\hline $\mathrm{H}$ & -5.19460200 & 1.30006000 & -0.34267600 \\
\hline $\mathrm{C}$ & -3.21272100 & 1.27621000 & 0.63568400 \\
\hline $\mathrm{C}$ & -2.68446800 & 2.56176100 & 0.45647500 \\
\hline $\mathrm{C}$ & -3.66733100 & 3.61279400 & 0.04403000 \\
\hline $\mathrm{C}$ & -3.54037500 & 4.30102500 & -1.18521000 \\
\hline $\mathrm{H}$ & -2.69616500 & 4.06723200 & -1.84143000 \\
\hline $\mathrm{C}$ & -4.49570700 & 5.24705400 & -1.57646100 \\
\hline $\mathrm{H}$ & -4.38287500 & 5.75672300 & -2.54065900 \\
\hline $\mathrm{C}$ & -5.60859000 & 5.54610200 & -0.76157800 \\
\hline $\mathrm{C}$ & -5.73830500 & 4.85079300 & 0.45728500 \\
\hline $\mathrm{H}$ & -6.59540600 & 5.06079200 & 1.10797200 \\
\hline $\mathrm{C}$ & -4.78929300 & 3.89776000 & 0.85343500 \\
\hline $\mathrm{H}$ & -4.90650500 & 3.37092400 & 1.80647000 \\
\hline $\mathrm{C}$ & -1.28485800 & 2.91693000 & 0.49408800 \\
\hline $\mathrm{C}$ & -0.86751500 & 4.30235300 & 0.34690400 \\
\hline $\mathrm{H}$ & -1.54101000 & 5.15684400 & 0.35455500 \\
\hline $\mathrm{C}$ & 0.48845400 & 4.31019000 & 0.18050600 \\
\hline $\mathrm{H}$ & 1.12703300 & 5.17323300 & 0.00842800 \\
\hline $\mathrm{C}$ & 0.93685200 & 2.92960300 & 0.26505700 \\
\hline $\mathrm{C}$ & 2.27523100 & 2.52201900 & 0.05362300 \\
\hline $\mathrm{C}$ & 3.20000400 & 3.63225000 & -0.36001400 \\
\hline $\mathrm{C}$ & 3.58277600 & 4.65570400 & 0.53664500 \\
\hline $\mathrm{H}$ & 3.21437700 & 4.62701100 & 1.56741300 \\
\hline $\mathrm{C}$ & 4.44089100 & 5.68587800 & 0.11980200 \\
\hline $\mathrm{H}$ & 4.73567000 & 6.46631000 & 0.82944800 \\
\hline $\mathrm{C}$ & 4.91946900 & 5.71600200 & -1.20072200 \\
\hline $\mathrm{H}$ & 5.58658000 & 6.52119900 & -1.52566500 \\
\hline $\mathrm{C}$ & 4.53414400 & 4.71018400 & -2.10280800 \\
\hline $\mathrm{H}$ & 4.89454800 & 4.73245500 & -3.13672500 \\
\hline $\mathrm{C}$ & 3.68348200 & 3.67510700 & -1.68691000 \\
\hline $\mathrm{H}$ & 3.38031200 & 2.89106800 & -2.38818400 \\
\hline $\mathrm{C}$ & 1.90950400 & 0.00029800 & 0.16681700 \\
\hline $\mathrm{N}$ & -0.15793800 & -2.10545100 & 0.50256400 \\
\hline $\mathrm{C}$ & -6.61540600 & -6.59675400 & -1.17854300 \\
\hline $\mathrm{H}$ & -7.58519500 & -6.44824000 & -0.67584200 \\
\hline $\mathrm{H}$ & -6.26429600 & -7.61315700 & -0.92013200 \\
\hline $\mathrm{H}$ & -6.78821400 & -6.58254900 & -2.26790300 \\
\hline $\mathrm{S}$ & -2.27246700 & -0.00051500 & 1.40010700 \\
\hline $\mathrm{N}$ & -0.15865900 & 2.10527700 & 0.50262200 \\
\hline $\mathrm{C}$ & -6.61767600 & 6.59488200 & -1.17694200 \\
\hline $\mathrm{H}$ & -7.58740000 & 6.44591000 & -0.67425200 \\
\hline $\mathrm{H}$ & -6.79051000 & 6.58089800 & -2.26630000 \\
\hline $\mathrm{H}$ & -6.26690200 & 7.61133800 & -0.91828100 \\
\hline $\mathrm{O}$ & 0.98669700 & -0.00018400 & 3.51613700 \\
\hline $\mathrm{C}$ & 0.57095700 & -0.00008200 & 2.41695400 \\
\hline
\end{tabular}

1-Ru(III) $)_{o}(\mathrm{uBP} 86 / 6-31 \mathrm{G}(\mathrm{d}, \mathrm{p}) / \mathrm{LANL} 2 \mathrm{DZ})$ 


\begin{tabular}{|c|c|c|c|}
\hline $\mathrm{Ru}$ & -0.09485700 & -0.00000800 & -0.40519900 \\
\hline $\mathrm{C}$ & 2.61904600 & 1.16543500 & 0.26526100 \\
\hline $\mathrm{C}$ & 3.87190800 & 0.73128300 & 0.87360900 \\
\hline $\mathrm{C}$ & 3.87194600 & -0.73105800 & 0.87361900 \\
\hline $\mathrm{C}$ & 2.61912200 & -1.16528300 & 0.26523600 \\
\hline $\mathrm{C}$ & 2.25713000 & -2.49211400 & -0.11992600 \\
\hline $\mathrm{C}$ & 0.93476800 & -2.89584900 & -0.43809800 \\
\hline $\mathrm{C}$ & 0.52814100 & -4.24320500 & -0.77139300 \\
\hline $\mathrm{C}$ & -0.84748800 & -4.25352100 & -0.79845600 \\
\hline $\mathrm{C}$ & -1.30255600 & -2.90628000 & -0.53230200 \\
\hline $\mathrm{C}$ & -2.70201700 & -2.57958500 & -0.34507400 \\
\hline $\mathrm{C}$ & -3.22937800 & -1.27834300 & -0.40016100 \\
\hline $\mathrm{C}$ & -4.36356500 & -0.69636300 & 0.24750100 \\
\hline $\mathrm{C}$ & -4.36361500 & 0.69604700 & 0.24750800 \\
\hline $\mathrm{C}$ & -3.22946300 & 1.27811000 & -0.40014000 \\
\hline $\mathrm{C}$ & -2.70219100 & 2.57938200 & -0.34500600 \\
\hline $\mathrm{C}$ & -1.30275300 & 2.90618600 & -0.53220200 \\
\hline $\mathrm{C}$ & -0.84778000 & 4.25348300 & -0.79824400 \\
\hline $\mathrm{C}$ & 0.52784900 & 4.24325700 & -0.77119700 \\
\hline $\mathrm{C}$ & 0.93457000 & 2.89590700 & -0.43800000 \\
\hline $\mathrm{C}$ & 2.25695800 & 2.49225300 & -0.11985100 \\
\hline $\mathrm{C}$ & 1.78773200 & 0.00005100 & 0.07803000 \\
\hline $\mathrm{N}$ & -0.19332900 & -2.08519400 & -0.37301700 \\
\hline $\mathrm{S}$ & -2.35572000 & -0.00008100 & -1.24153200 \\
\hline $\mathrm{N}$ & -0.19347000 & 2.08516800 & -0.37295100 \\
\hline $\mathrm{C}$ & 4.83015500 & 1.57513800 & 1.47932100 \\
\hline $\mathrm{C}$ & 6.00615200 & 1.25637300 & 2.17465100 \\
\hline $\mathrm{C}$ & 6.55354000 & 0.00019800 & 2.48020700 \\
\hline $\mathrm{C}$ & 6.00619700 & -1.25601100 & 2.17470800 \\
\hline $\mathrm{C}$ & 4.83022100 & -1.57485100 & 1.47937600 \\
\hline $\mathrm{C}$ & 3.31803300 & -3.53649700 & -0.28831800 \\
\hline $\mathrm{C}$ & 4.33166400 & -3.34579600 & -1.25531000 \\
\hline $\mathrm{C}$ & 5.33045500 & -4.31229200 & -1.44158600 \\
\hline $\mathrm{C}$ & 4.34134700 & -5.67886300 & 0.30625900 \\
\hline $\mathrm{C}$ & 3.33343800 & -4.71863300 & 0.48756200 \\
\hline $\mathrm{C}$ & 3.31778100 & 3.53672700 & -0.28818500 \\
\hline $\mathrm{C}$ & 3.33302800 & 4.71887400 & 0.48768200 \\
\hline $\mathrm{C}$ & 4.33148300 & 3.34610800 & -1.25511500 \\
\hline $\mathrm{C}$ & -3.63375400 & -3.67940000 & 0.02763100 \\
\hline $\mathrm{C}$ & -5.47158900 & -5.73967000 & 0.77224300 \\
\hline $\mathrm{C}$ & -5.79943100 & -4.79774100 & -0.22444300 \\
\hline $\mathrm{C}$ & -4.90214200 & -3.78688500 & -0.59111400 \\
\hline $\mathrm{C}$ & -3.63398600 & 3.67913700 & 0.02773300 \\
\hline $\mathrm{C}$ & -3.30692900 & 4.62140500 & 1.03292800 \\
\hline $\mathrm{C}$ & -5.47191200 & 5.73936500 & 0.77222400 \\
\hline $\mathrm{C}$ & -5.79969600 & 4.79739600 & -0.22458600 \\
\hline $\mathrm{C}$ & -4.90239700 & 3.78662600 & -0.59114800 \\
\hline $\mathrm{Cl}$ & -0.85631000 & -0.00007300 & 1.87288400 \\
\hline $\mathrm{C}$ & -6.42954600 & -6.85000200 & 1.14506200 \\
\hline $\mathrm{C}$ & -6.43008300 & 6.84972300 & 1.14440700 \\
\hline $\mathrm{H}$ & 1.19836800 & -5.08081400 & -0.95332700 \\
\hline $\mathrm{H}$ & -1.50194500 & -5.09859500 & -1.00629300 \\
\hline $\mathrm{H}$ & -5.07978800 & -1.30070400 & 0.80867800 \\
\hline $\mathrm{H}$ & -5.07987500 & 1.30033000 & 0.80869900 \\
\hline $\mathrm{H}$ & -1.50229700 & 5.09853000 & -1.00599600 \\
\hline $\mathrm{H}$ & 1.19801900 & 5.08092700 & -0.95306500 \\
\hline $\mathrm{H}$ & 4.62180200 & 2.64466100 & 1.41006400 \\
\hline
\end{tabular}




$\begin{array}{lrrr}\mathrm{H} & 6.57253600 & 2.12227300 & 2.53983000 \\ \mathrm{H} & 7.49636800 & 0.00022800 & 3.03939500 \\ \mathrm{H} & 6.57260900 & -2.12187300 & 2.53993100 \\ \mathrm{H} & 4.62191200 & -2.64438400 & 1.41016100 \\ \mathrm{H} & 2.56082100 & -4.86681700 & 1.24889200 \\ \mathrm{H} & 4.31747000 & 2.43842100 & -1.86669200 \\ \mathrm{H} & -6.77163800 & -4.86329400 & -0.72698900 \\ \mathrm{H} & -5.17040300 & -3.07613800 & -1.37972500 \\ \mathrm{H} & -6.77186600 & 4.86287300 & -0.72723100 \\ \mathrm{H} & -2.34546200 & 4.53851100 & 1.54892100 \\ \mathrm{H} & -5.17054900 & 3.07585500 & -1.37977700 \\ \mathrm{H} & 2.56034500 & 4.86700000 & 1.24895600 \\ \mathrm{H} & 4.34520600 & -6.58378100 & 0.92317900 \\ \mathrm{H} & 6.10173100 & -4.15416700 & -2.20275700 \\ \mathrm{H} & 4.31753100 & -2.43811700 & -1.86689600 \\ \mathrm{C} & 5.34157500 & -5.47932700 & -0.65941800 \\ \mathrm{C} & 4.34085800 & 5.67919600 & 0.30642600 \\ \mathrm{C} & 5.34116300 & 5.47973900 & -0.65918800 \\ \mathrm{C} & 5.33019700 & 4.31269300 & -1.44134200 \\ \mathrm{C} & -4.21072400 & 5.62709000 & 1.39498200 \\ \mathrm{C} & -3.30656900 & -4.62177500 & 1.03280400 \\ \mathrm{C} & -4.21023800 & -5.62743900 & 1.39490100 \\ \mathrm{H} & 6.12523000 & -6.23055500 & -0.80274900 \\ \mathrm{H} & 4.34459300 & 6.58412400 & 0.92333100 \\ \mathrm{H} & 6.12475600 & 6.23103900 & -0.80248300 \\ \mathrm{H} & 6.10153300 & 4.15463100 & -2.20246600 \\ \mathrm{H} & -3.93890400 & 6.33360700 & 2.18807400 \\ \mathrm{H} & -2.34502900 & -4.53887400 & 1.54866000 \\ \mathrm{H} & -3.93828200 & -6.33396200 & 2.18794900 \\ \mathrm{H} & -6.22853000 & -7.76484300 & 0.55678000 \\ \mathrm{H} & -7.47677800 & -6.56337200 & 0.95344100 \\ \mathrm{H} & -6.33765200 & -7.12310200 & 2.20956100 \\ \mathrm{H} & -7.47868800 & 6.55456900 & 0.97404000 \\ \mathrm{H} & -6.24422800 & 7.75673400 & 0.53924200 \\ \mathrm{H} & -6.32250000 & 7.13893700 & 2.20308000 \\ \mathrm{Cl} & 0.55845700 & 0.00004700 & -2.72416600\end{array}$

1-Ru(III) $)_{o}$ - EGS (uB3LYP/6-31G(d,p)/SDD)

$\begin{array}{crrr}\mathrm{Ru} & -0.09448800 & -0.00000200 & -0.43781100 \\ \mathrm{C} & 2.61037100 & 1.16036500 & 0.27257900 \\ \mathrm{C} & 3.86296200 & 0.73032500 & 0.86819400 \\ \mathrm{C} & 3.86297300 & -0.73027800 & 0.86819000 \\ \mathrm{C} & 2.61038800 & -1.16033300 & 0.27257200 \\ \mathrm{C} & 2.23826000 & -2.48366400 & -0.10619200 \\ \mathrm{C} & 0.92810600 & -2.88794500 & -0.42904700 \\ \mathrm{C} & 0.52002100 & -4.23445900 & -0.73772900 \\ \mathrm{C} & -0.84590800 & -4.24070100 & -0.77593800 \\ \mathrm{C} & -1.29462200 & -2.89293300 & -0.54337000 \\ \mathrm{C} & -2.69850900 & -2.55613000 & -0.37590200 \\ \mathrm{C} & -3.22302500 & -1.27716700 & -0.47928800 \\ \mathrm{C} & -4.39456600 & -0.68824900 & 0.10056600 \\ \mathrm{C} & -4.39457700 & 0.68817700 & 0.10056900 \\ \mathrm{C} & -3.22304500 & 1.27711500 & -0.47928200 \\ \mathrm{C} & -2.69854900 & 2.55608500 & -0.37588600 \\ \mathrm{C} & -1.29466600 & 2.89291200 & -0.54334500\end{array}$




\begin{tabular}{|c|c|c|c|}
\hline $\mathrm{C}$ & -0.84597400 & 4.24069100 & -0.77589100 \\
\hline $\mathrm{C}$ & 0.51995500 & 4.23447000 & -0.73768400 \\
\hline $\mathrm{C}$ & 0.92806200 & 2.88795800 & -0.42902100 \\
\hline $\mathrm{C}$ & 2.23822200 & 2.48369300 & -0.10617300 \\
\hline $\mathrm{C}$ & 1.78957700 & 0.00001100 & 0.07874300 \\
\hline $\mathrm{N}$ & -0.19808900 & -2.08292500 & -0.38740200 \\
\hline S & -2.33299500 & -0.00001700 & -1.29525400 \\
\hline $\mathrm{N}$ & -0.19812100 & 2.08291900 & -0.38738600 \\
\hline $\mathrm{C}$ & 4.82554200 & 1.56302500 & 1.45800600 \\
\hline $\mathrm{C}$ & 6.00803400 & 1.24736800 & 2.12925800 \\
\hline $\mathrm{C}$ & 6.55671700 & 0.00003800 & 2.42421200 \\
\hline $\mathrm{C}$ & 6.00805000 & -1.24729900 & 2.12925400 \\
\hline $\mathrm{C}$ & 4.82556300 & -1.56296800 & 1.45800000 \\
\hline $\mathrm{C}$ & 3.29268500 & -3.53565100 & -0.26340400 \\
\hline $\mathrm{C}$ & 4.27147900 & -3.38452000 & -1.25936600 \\
\hline $\mathrm{C}$ & 5.25787700 & -4.35424900 & -1.43738500 \\
\hline $\mathrm{C}$ & 4.32267200 & -5.64447300 & 0.37681600 \\
\hline $\mathrm{C}$ & 3.32707800 & -4.68103800 & 0.54911900 \\
\hline $\mathrm{C}$ & 3.29263100 & 3.53569700 & -0.26338200 \\
\hline $\mathrm{C}$ & 3.32701500 & 4.68107800 & 0.54915100 \\
\hline $\mathrm{C}$ & 4.27141900 & 3.38458900 & -1.25935400 \\
\hline $\mathrm{C}$ & -3.62398300 & -3.65019000 & 0.02962400 \\
\hline $\mathrm{C}$ & -5.43481300 & -5.68945500 & 0.82814600 \\
\hline $\mathrm{C}$ & -5.73220300 & -4.83919300 & -0.24326800 \\
\hline $\mathrm{C}$ & -4.84610800 & -3.83669300 & -0.63664700 \\
\hline $\mathrm{C}$ & -3.62403600 & 3.65013300 & 0.02964600 \\
\hline $\mathrm{C}$ & -3.32492200 & 4.50438900 & 1.10763900 \\
\hline $\mathrm{C}$ & -5.43491600 & 5.68933300 & 0.82821300 \\
\hline $\mathrm{C}$ & -5.73229500 & 4.83907300 & -0.24322400 \\
\hline $\mathrm{C}$ & -4.84617300 & 3.83662400 & -0.63663000 \\
\hline $\mathrm{Cl}$ & -0.84776200 & -0.00001600 & 1.83973200 \\
\hline $\mathrm{C}$ & -6.38424700 & -6.78589300 & 1.24843200 \\
\hline $\mathrm{C}$ & -6.38420700 & 6.78601900 & 1.24816900 \\
\hline $\mathrm{H}$ & 1.17984200 & -5.07440000 & -0.88967400 \\
\hline $\mathrm{H}$ & -1.49511500 & -5.08333000 & -0.96243800 \\
\hline $\mathrm{H}$ & -5.14169800 & -1.28476200 & 0.60974300 \\
\hline $\mathrm{H}$ & -5.14171800 & 1.28467700 & 0.60975000 \\
\hline $\mathrm{H}$ & -1.49519400 & 5.08331300 & -0.96237600 \\
\hline $\mathrm{H}$ & 1.17976200 & 5.07442400 & -0.88961700 \\
\hline $\mathrm{H}$ & 4.62253700 & 2.62326400 & 1.39849200 \\
\hline $\mathrm{H}$ & 6.57495900 & 2.10677300 & 2.48102500 \\
\hline $\mathrm{H}$ & 7.50104700 & 0.00004300 & 2.96298000 \\
\hline $\mathrm{H}$ & 6.57498500 & -2.10669800 & 2.48101800 \\
\hline $\mathrm{H}$ & 4.62257100 & -2.62321000 & 1.39848200 \\
\hline $\mathrm{H}$ & 2.57921700 & -4.80326700 & 1.32679000 \\
\hline $\mathrm{H}$ & 4.24462800 & 2.50739300 & -1.89869900 \\
\hline $\mathrm{H}$ & -6.66713600 & -4.96668100 & -0.78336400 \\
\hline $\mathrm{H}$ & -5.09236000 & -3.19841200 & -1.48010600 \\
\hline $\mathrm{H}$ & -6.66725700 & 4.96651400 & -0.78328600 \\
\hline $\mathrm{H}$ & -2.39579800 & 4.36757200 & 1.65178700 \\
\hline $\mathrm{H}$ & -5.09242400 & 3.19832200 & -1.48007300 \\
\hline $\mathrm{H}$ & 2.57915900 & 4.80328900 & 1.32682900 \\
\hline $\mathrm{H}$ & 4.34085300 & -6.51987200 & 1.01987400 \\
\hline $\mathrm{H}$ & 6.00098400 & -4.22742600 & -2.21948700 \\
\hline $\mathrm{H}$ & 4.24468100 & -2.50731800 & -1.89870400 \\
\hline $\mathrm{C}$ & 5.29000700 & -5.48446700 & -0.61733400 \\
\hline $\mathrm{C}$ & 4.32259400 & 5.64452900 & 0.37684800 \\
\hline
\end{tabular}




$\begin{array}{lrrr}\mathrm{C} & 5.28992300 & 5.48454400 & -0.61731200 \\ \mathrm{C} & 5.25780200 & 4.35433400 & -1.43737200 \\ \mathrm{C} & -4.21554600 & 5.49968600 & 1.49690900 \\ \mathrm{C} & -3.32482800 & -4.50449800 & 1.10758100 \\ \mathrm{C} & -4.21540700 & -5.49983200 & 1.49681400 \\ \mathrm{H} & 6.06173800 & -6.23652200 & -0.75379400 \\ \mathrm{H} & 4.34076800 & 6.51992200 & 1.01991300 \\ \mathrm{H} & 6.06164100 & 6.23661200 & -0.75377300 \\ \mathrm{H} & 6.00090500 & 4.22752700 & -2.21948200 \\ \mathrm{H} & -3.96511800 & 6.13690100 & 2.34190900 \\ \mathrm{H} & -2.39567100 & -4.36771700 & 1.65168100 \\ \mathrm{H} & -3.96491500 & -6.13711700 & 2.34174300 \\ \mathrm{H} & -5.97651600 & -7.77660000 & 1.01313400 \\ \mathrm{H} & -7.34889900 & -6.69672800 & 0.74127600 \\ \mathrm{H} & -6.56715200 & -6.76328200 & 2.32833200 \\ \mathrm{H} & -7.35080200 & 6.69353000 & 0.74531500 \\ \mathrm{H} & -5.97889500 & 7.77638000 & 1.00721100 \\ \mathrm{H} & -6.56257200 & 6.76743100 & 2.32888000 \\ \mathrm{Cl} & 0.60862500 & 0.00001200 & -2.73049400\end{array}$

1-Ru(III) $)_{\mathrm{o}}$ - EES (uB3LYP/6-31G(d,p)/SDD)

$\begin{array}{crrr}\mathrm{Ru} & 0.09653900 & -0.00001000 & -0.44114800 \\ \mathrm{C} & -2.60937900 & -1.15995900 & 0.27317000 \\ \mathrm{C} & -3.86179200 & -0.73071400 & 0.87024700 \\ \mathrm{C} & -3.86195800 & 0.73005100 & 0.87021800 \\ \mathrm{C} & -2.60960900 & 1.15953700 & 0.27314100 \\ \mathrm{C} & -2.23713100 & 2.48300500 & -0.10583300 \\ \mathrm{C} & -0.92703700 & 2.88741400 & -0.42858900 \\ \mathrm{C} & -0.51896300 & 4.23365000 & -0.73815400 \\ \mathrm{C} & 0.84718300 & 4.24013400 & -0.77565000 \\ \mathrm{C} & 1.29576900 & 2.89271300 & -0.54287900 \\ \mathrm{C} & 2.70016000 & 2.55574000 & -0.37655700 \\ \mathrm{C} & 3.22561300 & 1.27756300 & -0.48337700 \\ \mathrm{C} & 4.39945400 & 0.68861300 & 0.09232100 \\ \mathrm{C} & 4.39958400 & -0.68779900 & 0.09236000 \\ \mathrm{C} & 3.22585800 & -1.27700000 & -0.48330100 \\ \mathrm{C} & 2.70066100 & -2.55527900 & -0.37644800 \\ \mathrm{C} & 1.29633800 & -2.89250800 & -0.54275100 \\ \mathrm{C} & 0.84798600 & -4.24002000 & -0.77548200 \\ \mathrm{C} & -0.51815500 & -4.23377200 & -0.73796700 \\ \mathrm{C} & -0.92647500 & -2.88758800 & -0.42845400 \\ \mathrm{C} & -2.23663800 & -2.48339100 & -0.10574300 \\ \mathrm{C} & -1.78983400 & -0.00012900 & 0.07810200 \\ \mathrm{~N} & 0.19926100 & 2.08278800 & -0.38627500 \\ \mathrm{~S} & 2.33280400 & 0.00017000 & -1.29626200 \\ \mathrm{~N} & 0.19968100 & -2.08276900 & -0.38618900 \\ \mathrm{C} & -4.82268000 & -1.56325500 & 1.46195700 \\ \mathrm{C} & -6.00526600 & -1.24772600 & 2.13346300 \\ \mathrm{C} & -6.55420500 & -0.00058600 & 2.42784800 \\ \mathrm{C} & -6.00555100 & 1.24664900 & 2.13339600 \\ \mathrm{C} & -4.82302000 & 1.56240600 & 1.46187300 \\ \mathrm{C} & -3.29247400 & 3.53380400 & -0.26405700 \\ \mathrm{C} & -4.27543900 & 3.37689200 & -1.25503000 \\ \mathrm{C} & -5.26279900 & 4.34527100 & -1.43446700 \\ \mathrm{C} & -4.32116000 & 5.64541500 & 0.36922100\end{array}$




\begin{tabular}{|c|c|c|c|}
\hline $\mathrm{C}$ & -3.32453700 & 4.68323800 & 0.54283800 \\
\hline $\mathrm{C}$ & -3.29179200 & -3.53437500 & -0.26398100 \\
\hline $\mathrm{C}$ & -3.32365000 & -4.68382900 & 0.54289500 \\
\hline $\mathrm{C}$ & -4.27477300 & -3.37763700 & -1.25496600 \\
\hline $\mathrm{C}$ & 3.62399900 & 3.65016900 & 0.03162300 \\
\hline $\mathrm{C}$ & 5.42982900 & 5.69168000 & 0.83517300 \\
\hline $\mathrm{C}$ & 5.72918900 & 4.84480100 & -0.23937000 \\
\hline $\mathrm{C}$ & 4.84561100 & 3.84205100 & -0.63534600 \\
\hline $\mathrm{C}$ & 3.62473900 & -3.64952000 & 0.03169300 \\
\hline $\mathrm{C}$ & 3.32495700 & -4.49847000 & 1.11276800 \\
\hline $\mathrm{C}$ & 5.43108200 & -5.69062300 & 0.83513600 \\
\hline $\mathrm{C}$ & 5.73020900 & -4.84363900 & -0.23942000 \\
\hline $\mathrm{C}$ & 4.84638600 & -3.84111400 & -0.63533900 \\
\hline $\mathrm{Cl}$ & 0.84414700 & 0.00011300 & 1.83875200 \\
\hline $\mathrm{C}$ & 6.37285700 & 6.79673300 & 1.24699100 \\
\hline $\mathrm{C}$ & 6.37438000 & -6.79555100 & 1.24666800 \\
\hline $\mathrm{H}$ & -1.17879600 & 5.07326500 & -0.89182500 \\
\hline $\mathrm{H}$ & 1.49614500 & 5.08285900 & -0.96238400 \\
\hline $\mathrm{H}$ & 5.14898500 & 1.28491100 & 0.59814900 \\
\hline $\mathrm{H}$ & 5.14923400 & -1.28392300 & 0.59821600 \\
\hline $\mathrm{H}$ & 1.49709400 & -5.08263600 & -0.96219900 \\
\hline $\mathrm{H}$ & -1.17784100 & -5.07350500 & -0.89162100 \\
\hline $\mathrm{H}$ & -4.61888000 & -2.62344300 & 1.40406100 \\
\hline $\mathrm{H}$ & -6.57165100 & -2.10721100 & 2.48586500 \\
\hline $\mathrm{H}$ & -7.49849100 & -0.00067800 & 2.96668900 \\
\hline $\mathrm{H}$ & -6.57212900 & 2.10602900 & 2.48574700 \\
\hline $\mathrm{H}$ & -4.61945700 & 2.62263700 & 1.40391900 \\
\hline $\mathrm{H}$ & -2.57414300 & 4.80951100 & 1.31736900 \\
\hline $\mathrm{H}$ & -4.25069900 & -2.49651800 & -1.88893300 \\
\hline $\mathrm{H}$ & 6.66412900 & 4.97548000 & -0.77890500 \\
\hline $\mathrm{H}$ & 5.09333700 & 3.20646100 & -1.48042300 \\
\hline $\mathrm{H}$ & 6.66517000 & -4.97407100 & -0.77898400 \\
\hline $\mathrm{H}$ & 2.39744700 & -4.35600100 & 1.65819200 \\
\hline $\mathrm{H}$ & 5.09393000 & -3.20543800 & -1.48040300 \\
\hline $\mathrm{H}$ & -2.57324600 & -4.80996800 & 1.31743700 \\
\hline $\mathrm{H}$ & -4.33741000 & 6.52395700 & 1.00801800 \\
\hline $\mathrm{H}$ & -6.00897800 & 4.21385700 & -2.21286200 \\
\hline $\mathrm{H}$ & -4.25120600 & 2.49578600 & -1.88901000 \\
\hline $\mathrm{C}$ & -5.29211600 & 5.48001200 & -0.62049700 \\
\hline $\mathrm{C}$ & -4.32008000 & -5.64619800 & 0.36924300 \\
\hline $\mathrm{C}$ & -5.29105100 & -5.48096700 & -0.62048900 \\
\hline $\mathrm{C}$ & -5.26194200 & -4.34620400 & -1.43443600 \\
\hline $\mathrm{C}$ & 4.21410000 & -5.49488400 & 1.50492100 \\
\hline $\mathrm{C}$ & 3.32395500 & 4.49906300 & 1.11269800 \\
\hline $\mathrm{C}$ & 4.21283200 & 5.49566400 & 1.50490000 \\
\hline $\mathrm{H}$ & -6.06467200 & 6.23101500 & -0.75794200 \\
\hline $\mathrm{H}$ & -4.33616900 & -6.52475500 & 1.00802300 \\
\hline $\mathrm{H}$ & -6.06345700 & -6.23212000 & -0.75796300 \\
\hline $\mathrm{H}$ & -6.00813300 & -4.21492100 & -2.21284300 \\
\hline $\mathrm{H}$ & 3.96399600 & -6.12725600 & 2.35350700 \\
\hline $\mathrm{H}$ & 2.39643000 & 4.35639000 & 1.65804500 \\
\hline $\mathrm{H}$ & 3.96252300 & 6.12798700 & 2.35346600 \\
\hline $\mathrm{H}$ & 6.06894000 & 7.75854900 & 0.81476300 \\
\hline $\mathrm{H}$ & 7.39486700 & 6.59721200 & 0.91209600 \\
\hline $\mathrm{H}$ & 6.39016300 & 6.92346900 & 2.33412100 \\
\hline $\mathrm{H}$ & 7.39735400 & -6.59311200 & 0.91645900 \\
\hline $\mathrm{H}$ & 6.07383700 & -7.75629200 & 0.80969700 \\
\hline
\end{tabular}


$\begin{array}{llll}\mathrm{H} & 6.38765500 & -6.92619900 & 2.33337100\end{array}$

$\mathrm{Cl} \quad-0.60887700 \quad-0.00016800 \quad-2.73114300$ 